\title{
Consistent large-eddy simulation of a temporal mixing layer laden with evaporating drops. Part 1. Direct numerical simulation, formulation and $a$ priori analysis
}

\author{
By NORA A. OKONG'O AND JOSETTE BELLAN† \\ Jet Propulsion Laboratory, California Institute of Technology, Pasadena, CA 91109-8099, USA
}

(Received 8 August 2002 and in revised form 10 September 2003)

Large-eddy simulation (LES) models are presented and evaluated on a database obtained from direct numerical simulation (DNS) of a three-dimensional temporal mixing layer with evaporating drops. The gas-phase equations are written in an Eulerian frame for two perfect gas species (carrier gas and vapour emanating from the drops), while the liquid-phase equations are written in a Lagrangian frame. The effect of drop evaporation on the gas phase is considered through mass, momentum and energy source terms. The DNS database consists of transitional states attained by layers with different initial Reynolds numbers and initial liquid-phase mass loadings. Budgets of the LES equations at the transitional states show that, for the mass loadings considered, the filtered source terms (FSTs) are smaller than the resolved inviscid terms and some subgrid scale (SGS) terms, but larger than the resolved viscous stress, heat flux and mass flux terms. The irreversible entropy production (i.e. the dissipation) expression for a two-phase flow with phase change is derived, showing that the dissipation contains contributions due to viscous stresses, heat and species-mass fluxes, and source terms. For both the DNS and filtered flow fields at transition, the two leading contributions are found to be the dissipation due to the energy source term and that due to the chemical potential of the mass source. Therefore, the modelling effort is focused on both the SGS fluxes and the FSTs in the LES equations. The FST models considered are applicable to LES in which the grid is coarser than the DNS grid and, consistently, 'computational' drops represent the DNS physical drops. Because the unfiltered flow field is required for the computation of the source terms, but would not be available in LES, it was approximated using the filtered flow field or the filtered flow field augmented by corrections based on the SGS variances. All of the FST models were found to overestimate DNS-field FSTs, with the relative error of modelling the unfiltered flow field compared to the error of using computational drops showing a complex dependence on filter width and number of computational drops. For modelling the SGS fluxes and (where possible) SGS variances, constant-coefficient Smagorinsky, gradient and scale-similarity models were assessed on the DNS database, and calibrated coefficients were statistically equivalent when computed on single-phase or two-phase flows. The gradient and scale-similarity models showed excellent correlation with the SGS quantities. An a posteriori study is proposed to evaluate the impact of the studied models on the flow-field development, so as to definitively assess their suitability for LES with evaporating drops.

$\dagger$ Author to whom correspondence should be addressed: Josette.Bellan@jpl.nasa.gov 


\section{Introduction}

The accurate modelling of two-phase (TP) turbulent flows has been a long-standing problem that is so far unresolved. In these flows, the interaction of the phases through momentum, energy and sometimes mass transfer, determines the character of the flow. Because this interaction occurs at scales that are much smaller than the scale of practical interest, it is clear that routine simulations of such flows cannot embed a resolution of the physics at the interaction scales, indicating that phase interactions must be modelled.

Direct numerical simulation (DNS) is a methodology wherein all scales of the flow are resolved. For TP flows with particles that are much smaller than the Kolmogorov scale and which have a volumetrically small loading $\left(\simeq 10^{-3}\right)$, Boivin, Simonin \& Squires (1998) have shown that the drops can be treated as point sources of mass, momentum and energy from the gas-phase perspective; in such situations, it is appropriate to perform DNS using an Eulerian framework to describe the gas phase and a Lagrangian framework to track the drops. Although the volumetric loading is small, the mass loadings can be considerably larger $\left(\gtrsim 10^{-1}\right)$ owing to the ratio of liquid density to carrier gas density being $O\left(10^{3}\right)$, and therefore the drops may considerably influence the flow, i.e. two-way coupling. Since the drops are considered as point sources, the TP DNS resolution is essentially that which is adequate for single-phase (SP) flow. Recent studies using this DNS methodology, for isotropic turbulence, include those of Boivin et al. (1998) and Mashayek \& Jaberi (1999) for solid particles without phase change and those of Mashayek (1998a) and Réveillon \& Vervisch (2000) for evaporating drops. Other DNS with evaporating drops have been performed by Mashayek (1998b) for homogeneous shear flow and by Miller \& Bellan $(1999,2000)$ for temporal mixing layers.

Large-eddy simulation (LES) is a promising alternative to DNS. The LES gas-phase equations are derived by filtering the DNS gas-phase equations, leading to unclosed terms, which for SP flow are primarily the subgrid-scale (SGS) fluxes that arise from the convective terms. (The SGS fluxes appearing in the momentum equation are the SGS stresses.) For TP flows, the filtered source terms (FSTs) are also unclosed. Since only the large scales need to be resolved, LES may be conducted with reduced flowfield resolution compared to DNS. Consistent LES then means tracking a reduced number of drops; 'computational' drops are thus used to represent the physical drops. In this context, TP LES has additional modelling requirements compared to SP LES; whereas DNS requires modelling the interaction between individual drops and the unfiltered flow field, in LES neither the individual drops nor the unfiltered flow field are available and their interaction must be modelled from that between the filtered flow field and the computational drops.

Recent LES of TP flows include those by Deutsch \& Simonin (1991), Simonin, Deutsch \& Boivin (1993), Uijttewaal \& Oliemans (1996), Wang \& Squires (1996), Boivin, Simonin \& Squires (2000), and Yamamoto et al. (2001). These LES all considered an incompressible gas phase laden with small solid particles, with the particles not affecting the evolution of the gas phase, i.e. one-way coupling, and used physical or computational particles whose evolution was entirely governed by the resolved flow field. Wang \& Squires (1996) found SGS effects, included by modifying the gas-phase velocity felt by the particles, to be negligible. The LES of Boivin et al. (2000) and Yamamoto et al. (2001) included two-way coupling, but still neglected SGS effects on the particles. The SGS modelling requirement in these studies was confined to the gas phase, facilitating the use of the large body of work on SGS flux models for incompressible SP flow. Much less work has been devoted to compressible-flow SGS 
flux modelling and has mostly been confined to the energy equation in single-species SP flows (e.g. Erlebacher et al. 1992; Fureby 1996; Martin, Piomelli \& Candler 2000). SGS TP flow models that treated the drop contribution but did not reduce the size of the drop ensemble or consider the effect of the drops on the flow field were presented by Miller \& Bellan (2000) and Okong'o \& Bellan (2000).

In the present study, we seek to develop a consistent LES formulation for TP flows with evaporating drops, along with the necessary models for the unresolved quantities. To this end, we first perform DNS to create a database of transitional states upon which prospective models will be tested a priori. The DNS undertaken in this study generally follows the approach of Miller \& Bellan (1999, 2000). However, similar to Réveillon \& Vervisch (2000) and unlike Mashayek $(1998 a, b)$ or Miller \& Bellan (1999), we also include the effect of the species diffusional fluxes in the heat flux; this effect is important at the low temperature difference between gas and drops (compared to those typical of combustion problems) specified in the present DNS in order to foster interaction between drops and flow field. Next, we derive the LES equations suitable for our intended methodology and identify the terms that need to be modelled. Using the DNS database, we will present an analysis of these equations that justifies the focus of the present modelling effort on the FSTs and the SGS fluxes. The a priori analysis is similar in spirit to that of the compressible SP study of Vreman, Geurts $\&$ Kuerten (1995), albeit with different governing equations. Some of the models of Okong'o \& Bellan (2000) will be extended so as to calculate the FSTs from the filtered flow field and the reduced drop ensemble. Concerning the SGS fluxes, the emphasis will be on adapting and calibrating, for TP flows with phase change, models originally developed for incompressible SP LES; adaptation is necessary since describing TP flows requires additional conservation equations for energy and species mass and an equation of state. The required models depend on the form of the energy equation that is used (e.g. Erlebacher et al. 1992; Fureby 1996; Martin et al. 2000). The SGS flux models we shall consider are the Smagorinsky (see Smagorinksy 1993), scale-similarity (Bardina, Ferziger \& Reynolds 1980) and gradient (Clark, Ferziger \& Reynolds 1979) models. The application of these models to compressible flows is usually made through density-weighting. The thermodynamic variables are coupled through the equation of state, as well as through the heat and mass fluxes appearing in the energy and species equations. Therefore, appropriate modelling of the momentum, energy and species SGS fluxes must be carefully assessed.

This paper is organized as follows. In $\S 2$, we present the DNS formulation. This formulation consists of a set of coupled conservation equations for the gas phase and the liquid phase (i.e. the drops). The coupling describing the interaction between the two phases is provided in the gas-phase conservation equations by source terms. In $\S 3$, we analyse the DNS results; in particular, we evaluate the adopted form of the heat and mass fluxes. The gas-phase LES equations are developed in $\S 4$ and assumptions that may simplify these equations are examined using the DNS database. Thereafter, the contribution of the source terms is examined by considering the budget of the LES equations. The final form of the LES equations are then presented, in which only the SGS fluxes and the FSTs need be modelled. A parallel perspective on the importance of the source terms is presented in $\S 5$, wherein the irreversible entropy production (i.e. the dissipation) expression for two-phase flow with phase change is derived and evaluated for transitional DNS and the filtered flow fields. Models for the FSTs appearing in the LES equations are investigated in $\S 6$, while several constantcoefficient SGS models are proposed and calibrated in $\S 7$. The present study aims to create simple models so that the modelling approach can be validated by means of an 
a posteriori study. That study, to be undertaken in Part 2, will also address important modelling issues that cannot be examined a priori, such as spatial and temporal discretization errors, interpolation errors, the interaction of the models with the resolved scales, and determination of SGS model coefficients in the absence of a database for calibration. Finally, conclusions and further discussions are presented in $\S 8$.

\section{DNS equations}

The DNS equations are based on the formulation of Miller \& Bellan (1999). Here, we present only those aspects relevant to the LES methodology designed to replicate the DNS large scales. The governing equations are formulated in an Eulerian frame for the gas phase and a Lagrangian frame for the drops. The gas-phase equations are formulated for two species, namely the carrier gas and the vapour evolving from the drops. The drops are treated as point sources of mass, momentum and energy for the gas phase; this treatment is justified by the dilute (i.e. volumetrically small, $O\left(10^{-3}\right)$ ) loading and the size of each particle being much smaller than the Kolmogorov scale. Each drop is assumed to be spherical, and, consistent with the drop description as a point source, its internal temperature and density are assumed uniform. Furthermore, we neglect unsteady drag and added mass effects, as well as Basset history forces, all of which are small for liquid-to-gas density ratios (Boivin et al. 1998). Drop collisions are assumed negligible.

\subsection{Gas-phase equations}

For ease of notation, we define the vector of gas-phase conservative variables $\phi=$ $\left\{\rho, \rho u_{i}, \rho e_{t}, \rho Y_{V}\right\}$ and denote the flow field as $\phi$, where $\rho$ is the density, $u_{i}$ is the velocity in the $x_{i}$ coordinate direction, $e_{t}$ is the total energy and $Y_{V}$ is the vapour (subscript $V$ ) mass fraction (the carrier gas, subscript $C$, mass fraction is $Y_{C}$; $\left.Y_{C}+Y_{V}=1\right)$. The gas-phase conservation equations are:

$$
\begin{gathered}
\frac{\partial \rho}{\partial t}+\frac{\partial\left(\rho u_{j}\right)}{\partial x_{j}}=S_{I}, \\
\frac{\partial\left(\rho u_{i}\right)}{\partial t}+\frac{\partial\left(\rho u_{i} u_{j}\right)}{\partial x_{j}}=-\frac{\partial p}{\partial x_{i}}+\frac{\partial \sigma_{i j}}{\partial x_{j}}+S_{I I, i}, \\
\frac{\partial\left(\rho e_{t}\right)}{\partial t}+\frac{\partial\left(\rho e_{t} u_{j}\right)}{\partial x_{j}}=-\frac{\partial\left(p u_{j}\right)}{\partial x_{j}}-\frac{\partial q_{j}}{\partial x_{j}}+\frac{\partial\left(\sigma_{i j} u_{i}\right)}{\partial x_{j}}+S_{I I I}, \\
\frac{\partial\left(\rho Y_{V}\right)}{\partial t}+\frac{\partial\left(\rho Y_{V} u_{j}\right)}{\partial x_{j}}=-\frac{\partial j_{V j}}{\partial x_{j}}+S_{I},
\end{gathered}
$$

where $S_{I}, S_{I I, i}$ and $S_{I I I}$ are source terms due to the action of the drops. The thermodynamic variables to be computed from $\phi$ are the internal energy $\left(e=e_{t}-e_{k}\right.$, where the kinetic energy is $\left.e_{k}=u_{i} u_{i} / 2\right)$, the pressure $(p)$, the temperature $(T)$ and the enthalpy $(h=e+p / \rho)$. We assume perfect gases, for which

$$
p(\phi)=\rho R(\phi) T(\phi),
$$

where $R(\phi)=Y_{V} R_{V}+Y_{C} R_{C}, R_{V}=R_{u} / m_{V}, R_{C}=R_{u} / m_{C}, R_{u}$ is the universal gas constant and $m_{C}$ and $m_{V}$ are the molar weights of the carrier gas and vapour, respectively, and

$$
h(\phi)=h_{V} Y_{V}+h_{C} Y_{C},
$$


where $h_{C}$ and $h_{V}$ are the enthalpies of the pure gases,

$$
h_{C}=\int C_{p, C}(T) \mathrm{d} T, \quad h_{V}=\int C_{p, V}(T) \mathrm{d} T,
$$

which are calculated from a given functional form of the heat capacities at constant pressure, $C_{p, C}$ and $C_{p, V}$. For the small temperature and pressure range to be simulated, we assume the gas to be calorically perfect, meaning that $C_{p, C}$ and $C_{p, V}$ are constant; then

$$
h_{C}(\phi)=C_{p, C} T, \quad h_{V}(\phi)=C_{p, V} T+h_{V}^{0},
$$

where $C_{p, C}=C_{p, C}\left(T^{0}\right), C_{p, V}=C_{p, V}\left(T^{0}\right)$ and $h_{V}^{0}$ is the reference vapour enthalpy at $\left(T^{0}, p^{0}\right)$ obtained from integration or tables, which accounts for the enthalpy difference between the vapour and carrier gas at the reference conditions. Then (2.6) becomes

$$
h(\phi)=C_{p}(\phi) T(\phi)+h_{V}^{0} Y_{V}
$$

where the mixture heat capacity at constant pressure is $C_{p}(\phi)=C_{p, V} Y_{V}+C_{p, C} Y_{C}$. The temperature $T$ is computed from the internal energy through

$$
e(\phi)=C_{v}(\phi) T(\phi)+h_{V}^{0} Y_{V}
$$

where $C_{v}$ is the mixture heat capacity at constant temperature $\left(C_{v}=C_{p}-R\right)$.

For (2.1)-(2.4), we specify the following functions of the flow field: the viscous stress,

where $\mu$ is the viscosity and

$$
\sigma_{i j}(\phi)=2 \mu\left(S_{i j}-\frac{1}{3} S_{k k} \delta_{i j}\right),
$$

$$
S_{i j}(\phi)=\frac{1}{2}\left(\frac{\partial u_{i}}{\partial x_{j}}+\frac{\partial u_{j}}{\partial x_{i}}\right)
$$

is the rate of strain; the vapour mass flux,

$$
j_{V j}(\phi)=\rho Y_{V} V_{V j}(\phi)=-\rho Y_{V}\left[\frac{D}{Y_{V}} \frac{\partial Y_{V}}{\partial x_{j}}+Y_{C}\left(Y_{V}+Y_{C} \frac{m_{V}}{m_{C}}\right)\left[\frac{m_{C}}{m_{V}}-1\right] \frac{D}{p(\phi)} \frac{\partial p(\phi)}{\partial x_{j}}\right],
$$

where thermal diffusion effects have been neglected, $V_{V j}$ is the vapour diffusion velocity, and $D$ is the diffusion coefficient; the carrier gas mass flux

$$
j_{C j}(\phi)=\rho Y_{C} V_{C j}(\phi)=-j_{V j}
$$

and the heat flux,

$$
q_{j}(\phi)=-\lambda \frac{\partial T(\phi)}{\partial x_{j}}+\left(h_{V}(\phi)-h_{C}(\phi)\right) j_{V j}(\phi),
$$

where $\lambda$ is the thermal conductivity. In (2.11), (2.13) and (2.15), $\mu, D$ and $\lambda$ are assumed constant, and may be defined through the Prandtl and Schmidt numbers, $\operatorname{Pr}=\mu C_{p} / \lambda$ and $S c=\mu /(\rho D)$. In Miller \& Bellan $(1999,2000)$, the Fick contribution was assumed to dominate the diffusional fluxes, and thus the second (pressure gradient) term was neglected in (2.13); also, the Fourier term was assumed to dominate the heat flux and the second (enthalpy flux) term in (2.15) was not included.

\subsection{Drop (liquid-phase) equations}

The equations describing the drop evolution have been presented in detail by Miller $\&$ Bellan (1999) and only their essence is presented here. We define $Z=\left\{X_{i}, v_{i}, T_{d}, m_{d}\right\}$ 
as the drop field with position $X_{i}$, velocity $v_{i}$, temperature $T_{d}$, and mass $m_{d}$. Under the assumptions stated previously, the evolution equations for the drops, in a Lagrangian frame, are:

$$
\begin{gathered}
\frac{\mathrm{d} X_{i}}{\mathrm{~d} t}(Z)=v_{i}, \\
\frac{\mathrm{d} v_{i}}{\mathrm{~d} t}\left(\psi_{f}, Z\right)=\frac{1}{m_{d}} F_{i}\left(\psi_{f}, Z\right), \\
\frac{\mathrm{d} T_{d}}{\mathrm{~d} t}\left(\psi_{f}, \psi_{s}, Z\right)=\frac{1}{m_{d} C_{L}}\left[Q\left(\psi_{f}, Z\right)+\dot{m}_{d}\left(\psi_{f}, \psi_{s}, Z\right) L_{V}(Z)\right], \\
\frac{\mathrm{d} m_{d}}{\mathrm{~d} t}\left(\psi_{f}, \psi_{s}, Z\right)=\dot{m}_{d}\left(\psi_{f}, \psi_{s}, Z\right),
\end{gathered}
$$

where $F_{i}$ is the drag force, $Q$ is the heat flux, $\dot{m}_{d}$ is the evaporation rate, and $C_{L}$ is the heat capacity of the drop liquid. $L_{V}$ is the latent heat of vaporization, which, for calorically perfect gases, is a linear function of temperature, $L_{V}=h_{V}^{0}-\left(C_{L}-C_{p, V}\right) T_{d}$. The drop evolution depends on the gas-phase primitive variables, $\psi(\phi)=\left\{u_{i}, T, Y_{V}, p\right\}$, evaluated either at the drop surface (subscript $s$ ) or at the drop far-field (subscript $f$ ). The far-field variables are taken as the gas-phase primitive variables interpolated to the drop locations. The detailed expressions for $F_{i}, Q$ and $\dot{m}_{d}$ involve validated correlations for point drops which are based on Stokes drag, with the particle time constant defined by Crowe, Chung \& Troutt (1998) as $\tau_{d}=\rho_{L} d^{2} /(18 \mu)$, where $\rho_{L}$ is the density of the liquid and $d$ is the drop diameter $\left(m_{d}=\rho_{L} \pi d^{3} / 6\right)$ :

$$
\begin{gathered}
F_{i}\left(\psi_{f}, Z\right)=\frac{m_{d}}{\tau_{d}} f_{1}\left(u_{i, f}-v_{i}\right), \\
Q\left(\psi_{f}, Z\right)=\frac{m_{d}}{\tau_{d}} \frac{N u}{3 P r} C_{p, f} f_{2}\left(T_{f}-T_{d}\right), \\
\dot{m}_{d}\left(\psi_{f}, Z\right)=-\frac{m_{d}}{\tau_{d}} \frac{S h}{3 S c} \ln \left[1+B_{M}\right] .
\end{gathered}
$$

In (2.20), $f_{1}$ is an empirical correlation to correct the Stokes drag for finite drop Reynolds numbers

$$
\begin{gathered}
f_{1}=\frac{1+0.0545 R e_{s l}+0.1 R e_{s l}^{1 / 2}\left(1-0.03 R e_{s l}\right)}{1+a\left|R e_{b}\right|^{b}}, \\
a=0.09+0.077 \exp \left(-0.4 R e_{s l}\right), \quad b=0.4+0.77 \exp \left(-0.04 R e_{s l}\right),
\end{gathered}
$$

based on the slip Reynolds number $R e_{s l}=\left|u_{i, f}-v_{i}\right| \rho d / \mu$, where $u_{i, f}-v_{i}$ is the slip velocity, and on the blowing Reynolds number $\operatorname{Re}_{b}=U_{b} \rho d / \mu$, where $U_{b}$ is the blowing velocity obtained from the mass conservation relation at the drop surface, $\dot{m}_{d}=-\pi \rho d^{2} U_{b}$. The correlation of (2.23) is valid for the ranges $0 \leqslant R e_{s l} \leqslant 100$ and $0 \leqslant R e_{b} \leqslant 10$. In (2.21), $C_{p, f}=C_{p, V} Y_{V, f}+C_{p, C} Y_{C, f}$ and $f_{2}=\beta /\left(e^{\beta}-1\right)$, where $\beta=-1.5 \operatorname{Pr} \dot{m}_{d} \tau_{d} / m_{d}$ is constant for drops obeying the classical ' $d^{2}$ law' (Williams 1965). In (2.22), the mass transfer number is $B_{M}=\left(Y_{V, s}-Y_{V, f}\right) /\left(1-Y_{V, s}\right)$ where $Y_{V, s}$ is calculated directly from the surface vapour mole fraction, $X_{V, s}$, which is obtained by equating the vapour and liquid fugacities at the surface (i.e. $X_{V, s} p_{s}=p_{\text {sat }}$; also $p_{s}=p_{f}$ ), where the saturation pressure, $p_{s a t}$, is provided by the Clausius-Clapeyron relation. Therefore,

$$
X_{V, s}=\frac{p_{a t m}}{p_{s}} \exp \left[\frac{L_{V}}{R_{V}}\left(\frac{1}{T_{B, L}}-\frac{1}{T_{d}}\right)\right], \quad Y_{V, s}=\frac{X_{V, s}}{X_{V, s}+\left(1-X_{V, s}\right) m_{C} / m_{V}},
$$


where $p_{a t m}=1$ atm and $T_{B, L}$ is the liquid saturation temperature at $p_{a t m}$ (i.e. the normal boiling temperature). Finally, the Nusselt, $N u$, and Sherwood, Sh, numbers appearing in (2.21) and (2.22) are empirically modified for convective corrections to heat and mass transfer based on the Ranz-Marshall correlations

$$
N u=2+0.552 \operatorname{Re}_{s l}^{1 / 2}(\operatorname{Pr})^{1 / 3}, \quad S h=2+0.552 R e_{s l}^{1 / 2}(S c)^{1 / 3} .
$$

Except for $\tau_{d}$, which depends on $\mu,(2.20)-(2.22)$ depend essentially on ratios of transport properties through non-dimensional numbers. Therefore, the value of $\tau_{d}$ and thus for a given liquid and drop size, the value of $\mu$ determines the interaction time between drops and gas.

\subsection{Source terms}

As in Miller \& Bellan (1999), each drop acts as a point source of mass, momentum and energy for the gas phase, with the drop source vector

$$
\begin{gathered}
S_{d}\left(\psi_{f}, Z\right)=\left\{S_{I, d}, S_{I I, i, d}, S_{I I I, d}\right\}, \\
S_{I, d}=-\dot{m}_{d}, \\
S_{I I, i, d}=-\left[F_{i}+\dot{m}_{d} v_{i}\right], \\
S_{I I I, d}=-\left[F_{i} v_{i}+Q+\dot{m}_{d}\left(\frac{1}{2} v_{i} v_{i}+h_{V, s}\right)\right],
\end{gathered}
$$

where $h_{V, s}=C_{p, V} T_{d}+h_{V}^{0}$ is the vapour enthalpy at the drop surface. The drop sources in the Lagrangian frame must be reconstructed in the Eulerian frame to obtain the gasphase source vector $S$ which contains the terms in (2.1)-(2.4), S( $\left.\psi_{f}, Z\right)=\left\{S_{I}, S_{I I, i}, S_{I I I}\right\}$. As in Miller \& Bellan (1999), we use

$$
S\left(\psi_{f}, Z\right)=\sum_{\alpha} \frac{w_{\alpha}}{V}\left[S_{d}\left(\psi_{f}, Z\right)\right]_{\alpha},
$$

where the summation is over all drops $\alpha$ residing within a local numerical discretization volume, $V$, and the geometrical weighting factor, $w_{\alpha}$, is used to distribute the individual drop contributions to the eight nearest neighbour surrounding grid points (i.e. corners of the computational volume $V$ ) proportionally to the drop distance from those nodes. These source terms are then minimally 'smoothed' using a conservative operator so as to retain numerical stability of the Eulerian gas-phase fields (see Miller \& Bellan 1999). This smoothing is not a filter in that it does not remove flow scales, but is required for successful simulations due to the 'spottiness' of the source terms.

\subsection{Flow configuration and numerical procedure}

The mixing-layer geometric configuration is illustrated in figure 1, where the streamwise $\left(x_{1}\right)$, the cross-stream $\left(x_{2}\right)$, and the spanwise $\left(x_{3}\right)$ coordinates are shown, and the domain lengths are $L_{1}, L_{2}$ and $L_{3}$ in each direction. Periodic boundary conditions are used in the $x_{1}$ - and $x_{3}$-directions, and adiabatic slip wall conditions (see Poinsot $\&$ Lele 1992) are employed for the $x_{2}$ boundaries. Drops reaching the slip walls are assumed to stick to the wall, but are otherwise transported according to the drop evolution equations (2.16)-(2.19). Initially, the gas phase consists only of the carrier gas (no vapour). The free-stream velocity $U_{0}=M_{c, 0} a_{C, 0}$ is calculated from a specified value of the convective Mach number $M_{c, 0}$ based on the carrier gas initial speed of sound $a_{C, 0}=\sqrt{R_{C} T_{C, 0} C_{p, C} / C_{v, C}}$ where $T_{C, 0}$ is the initial uniform temperature of the carrier gas at the initial uniform pressure. The initial vorticity thickness is $\delta_{\omega, 0}=\delta_{\omega}(0)$ where $\delta_{\omega}(t)=\Delta U_{0} /\left(\partial\left\langle u_{1}\right\rangle / \partial x_{2}\right)_{\max }$, with the brackets \langle\rangle denoting averaging over homogeneous $\left(x_{1}, x_{3}\right)$ planes and $\Delta U_{0}=2 U_{0}$ being the velocity difference across 


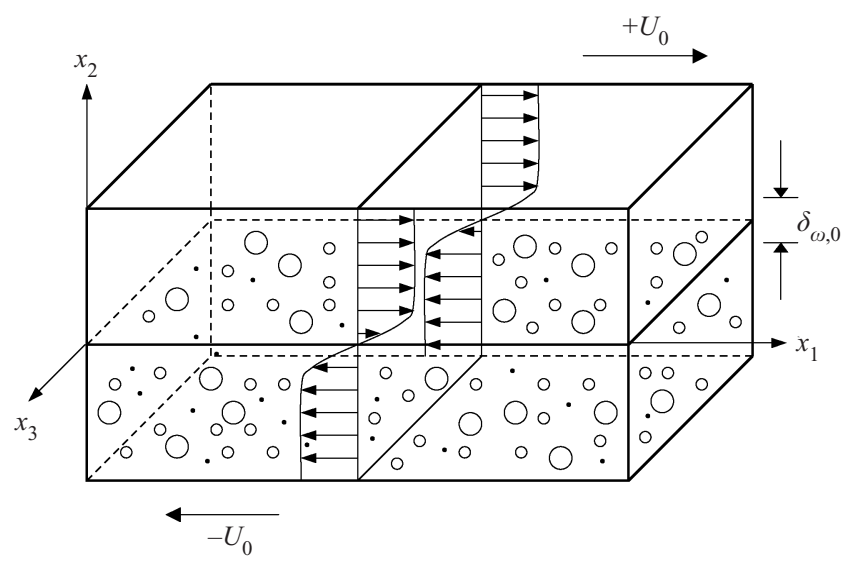

FIGURE 1. Mixing-layer configuration.

the layer; the initial mean streamwise velocity has an error-function profile. The specified value of the initial Reynolds number, $R e_{0}=\rho_{0} \Delta U_{0} \delta_{\omega, 0} / \mu$, where $\rho_{0}$ is the initial gas density, is used to calculate $\mu$. The thermal conductivity and diffusivity are then computed using this value of $\mu$ and (constant) specified values of Prandtl and Schmidt numbers. All thermophysical properties are the same as those employed in the simulations of Miller \& Bellan (2000) using air as the carrier gas and decane as the drop liquid.

To promote layer growth, the layer is initially perturbed so as to induce roll-up and pairing. The perturbations, described in Miller \& Bellan (1999), follow Moser \& Rogers (1991) in specifying spanwise and streamwise vorticity fluctuations, with streamwise and spanwise wavelengths in the $x_{1}$ - and $x_{3}$-directions of $\lambda_{1}=7.29 \delta_{\omega, 0}$ and $\lambda_{3}=0.6 \lambda_{1}$. For all the simulations performed herein, $L_{1}=4 \lambda_{1}, L_{2}=1.1 L_{1}$ and $L_{3}=4 \lambda_{3}$. The relative amplitudes of the forcing perturbations with respect to the circulations are $10 \%$ and $2.25 \%$ in the spanwise and streamwise directions, respectively. The evolution of the layer comprises two pairings for the four initial spanwise vortices to form a single vortex.

The drops are initially distributed randomly throughout the $x_{2}<0$ domain with specified temperature, velocity, number density and size distribution. Initially, all the drops have the same temperature, $T_{d, 0}$, which is lower than $T_{C, 0}$ and $T_{B, L}$ to promote evaporation; the initial velocity of each drop is the same as that of the gas phase at its location. The mean number density profile is smoothed near the centreline, $x_{2}=0$, using an error function profile. The drop size distribution is specified through the drop Stokes number $S t=\tau_{d} \Delta U_{0} / \delta_{\omega, 0}$, which is initially given by a Gaussian distribution (see table 1). The initial number of drops is determined by the initial mass loading $M L_{0}$ (initial ratio of liquid mass to the mass of carrier gas in the drop-laden part of domain).

The equations are solved numerically using a fourth-order explicit Runge-Kutta temporal integration for time derivatives and an eighth-order central finite differencing with tenth-order filtering for spatial derivatives (Kennedy \& Carpenter 1994). This filtering introduces a small amount of dissipation that serves only to stabilize the computations for long-time integrations, but since it acts only on the shortest waves that can be resolved on the grid, it does not act as a turbulence model allowing underresolved computations (Kennedy \& Carpenter 1994). A fourth-order Lagrange interpolation procedure is used to obtain gas-phase variable values at the drop locations. 


\begin{tabular}{|c|c|c|c|c|c|c|c|c|}
\hline Run & $R e_{0}$ & $M L_{0}$ & $N_{d}$ & $N_{1} \times N_{2} \times N_{3}$ & $t_{\text {trans }}^{*}$ & $\begin{array}{l}\delta_{m} / \delta_{\omega, 0} \\
\text { at } t_{\text {trans }}^{*}\end{array}$ & $\begin{array}{l}R e_{m} \\
\text { at } t_{\text {trans }}^{*}\end{array}$ & $\begin{array}{l}\text { CPU-hours } \\
\text { (estimated) }\end{array}$ \\
\hline SP500 & 500 & 0 & 0 & $256 \times 288 \times 160$ & 100 & 2.580 & 1290 & 688 \\
\hline ТP500a2 & 500 & 0.2 & 2277222 & $56 \times 288 \times 160$ & 1 & 2.729 & 136 & 1586 \\
\hline TP500a5 & 500 & 0.5 & 5693055 & $56 \times 288 \times 160$ & 105 & 2.722 & & 2075 \\
\hline SP600 & 600 & 0 & 0 & 288 & 10 & & & 1054 \\
\hline ТР60 & 600 & & 993630 & & & & & \\
\hline TP600a5 & 600 & 0.5 & 7484075 & $288 \times 320 \times 176$ & 105 & 2.613 & 1568 & 2981 \\
\hline \multicolumn{9}{|c|}{$\begin{array}{l}R e_{0}: \text { initial-vorticity-thickness Reynolds number, } R e_{0}=\rho_{0} \Delta U_{0} \delta_{\omega, 0} / \mu \\
M L_{0}: \text { initial mass loading } \\
N_{d}: \text { initial number of drops } \\
N_{1}, N_{2}, N_{3}: \text { number of grid points in } x_{1}, x_{2} \text { and } x_{3} \text { directions, respectively } \\
t_{\text {trans }}^{*} \text { transition time (dimensionless, rounded to nearest } t^{*} \text { divisible by } 5 \text { ), } t^{*}=t \Delta U_{0} / \delta_{\omega, 0} \\
R e_{m}: \text { momentum-thickness Reynolds number, } R e_{m}=\rho_{0} \Delta U_{0} \delta_{m} / \mu \\
\text { CPU hours are estimates on } 64 \text { processors of an SGI Origin } 2000 \\
\text { For all cases: } L_{1}=0.2 \mathrm{~m}, L_{2}=0.22 \mathrm{~m}, L_{3}=0.12 \mathrm{~m}, M_{c, 0}=0.35, T_{C, 0}=375 \mathrm{~K}, \rho_{0}=0.9415 \mathrm{~kg} \mathrm{~m}^{-3} \text {, } \\
\quad \Delta U_{0}=271.7 \mathrm{~m} \mathrm{~s}^{-1}, \delta_{\omega, 0}=6.859 \times 10^{-3} \mathrm{~m} \\
\text { For drop laden cases: } T_{d, 0}=345 \mathrm{~K}, T_{B, L}=447.7 \mathrm{~K}, \rho_{L}=642 \mathrm{~kg} \mathrm{~m}^{-3} \text {; the initial } S t \text { has a Gaussian } \\
\quad \text { distribution with mean } 3 \text { and standard deviation } 0.5\end{array}$} \\
\hline
\end{tabular}

TABLE 1. Initial conditions and transition times.

\section{DNS results}

The DNS described herein was undertaken to achieve several transitional states that could be further analysed for a priori turbulence modelling. All DNS initial conditions and respective resolutions are given in table 1 . Four of the cases have initial conditions identical to those of Miller \& Bellan (2000); two additional $R e_{0}=600$ cases, one (SP600) with no drops and another (TP600a5) with a higher initial mass loading $\left(M L_{0}=0.5\right)$, have been simulated. As discussed in $\S 2.1$, compared to Miller \& Bellan (2000), the gas-phase equations have been expanded to account for enthalpy flux and pressure diffusion effects, with corresponding changes in the boundary conditions. Further, the simulations have been performed for longer non-dimensional times compared to those in Miller \& Bellan (2000). The rationale behind conducting both TP and SP DNS is that, although the focus is on TP DNS, it is desirable to perform as much gas-phase model development as possible on the SP database. The more of the SP information we can use for TP modelling, the more we can take advantage of the existing body of work pertaining to SP LES modelling and focus our efforts on uniquely drop-related modelling issues. The resolution for the TP cases is the same as that for the SP cases at the same $R e_{0}$, and is varied approximately linearly with $R e_{0}$. The adequacy of the resolutions is assessed by computing one-dimensional energy spectra at the transitional times with the plots in figure 2 for TP600a2 (streamwise spectra, figure $2 a$; spanwise spectra, figure $2 b$ ) being typical. These plots show that most of the energy is in the large scales (low wavenumber, $k$ ) and that there is no accumulation of energy at high wavenumbers.

To understand the characteristics of the mixing layers, we analyse their global and detailed characteristics.

\subsection{Global layer evolution}

The global quantities for the layers are plotted versus the non-dimensional time $t^{*}=t \Delta U_{0} / \delta_{\omega, 0}$ in figure 3. Among the various quantities available to measure the 

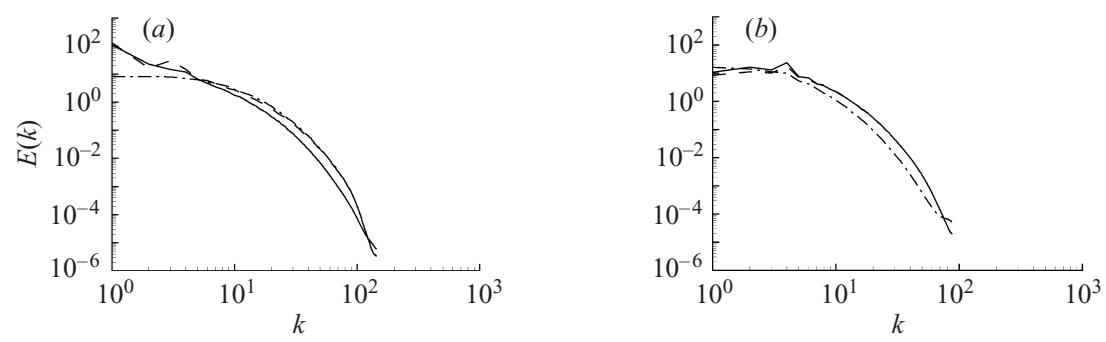

FIGURE 2. One-dimensional energy spectra, TP600a2, $t^{*}=105:(a)$ streamwise, $(b)$ spanwise. ,$- u_{1} ;---, u_{2} ;-\cdot-, u_{3}$.
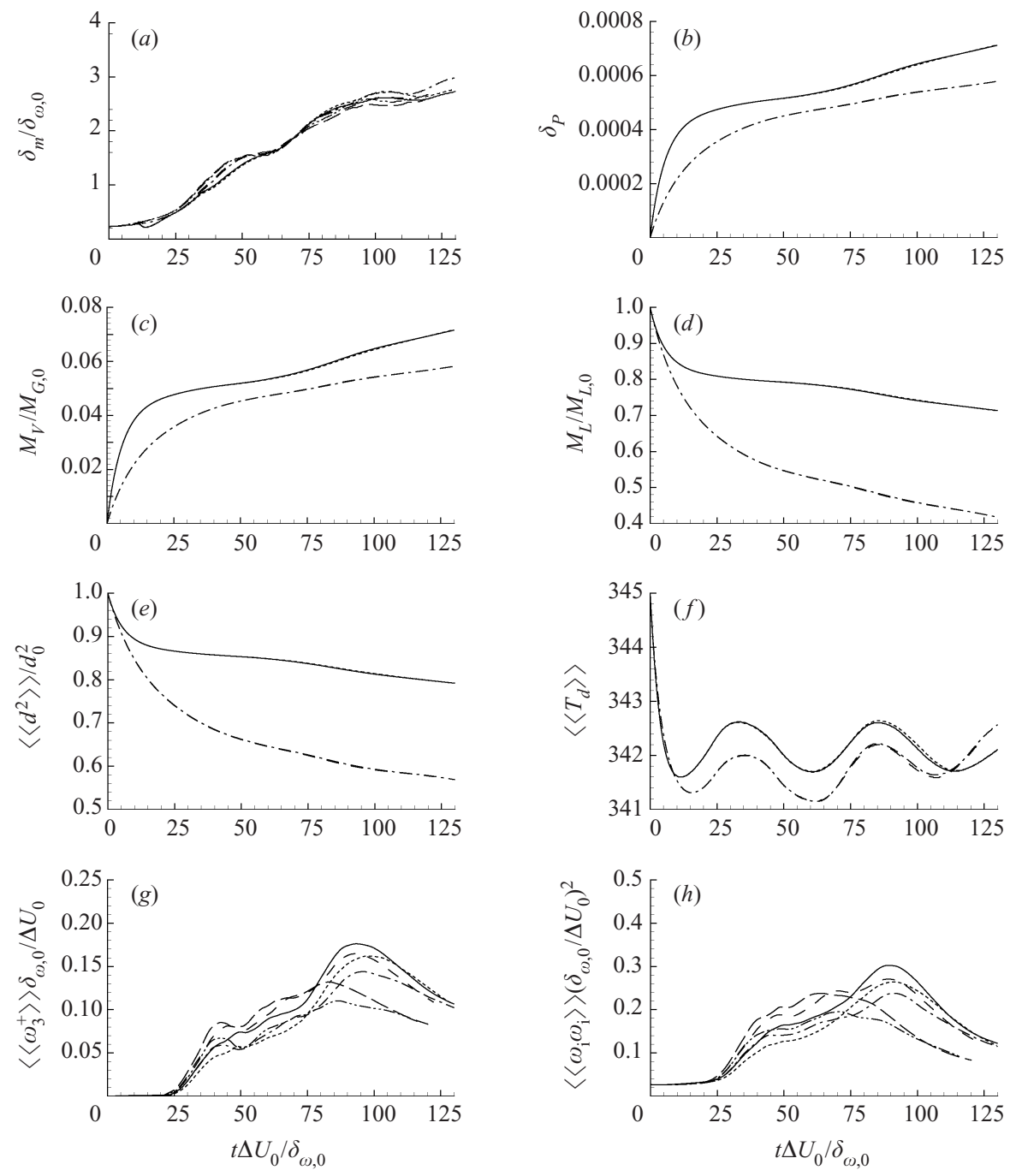

FIgURE 3. Time evolution of global quantities: $(a)$ momentum thickness, $(b)$ product thickness $(\mathrm{kg}),(c)$ mass of vapour, $(d)$ mass of liquid, $(e)$ average drop diameter-squared, $(f)$ average drop temperature $(\mathrm{K}),(g)$ average positive spanwise vorticity and $(h)$ enstrophy; $(a, g, h)$ for all layers, $(b-f)$ for drop-laden layers. $-. \cdot-$, SP500; ---, TP500a2; -.-, TP500a5; - - SP600; --- , TP600a2; -, TP600a5. 
layer growth, we adopt the momentum thickness $\delta_{m}$, calculated as

$$
\delta_{m}=\frac{\int_{x_{2, \min }}^{x_{2, \max }}\left[\left\langle\rho u_{1}\right\rangle_{x_{2, \max }}-\left\langle\rho u_{1}\right\rangle\right]\left[\left\langle\rho u_{1}\right\rangle-\left\langle\rho u_{1}\right\rangle_{x_{2, \text { min }}}\right] \mathrm{d} x_{2}}{\left(\left\langle\rho u_{1}\right\rangle_{x_{2, \max }}-\left\langle\rho u_{1}\right\rangle_{x_{2, \min }}\right)^{2}},
$$

where $x_{2, \max }=L_{2} / 2$ and $x_{2, \min }=-L_{2} / 2$ are the slip wall coordinates. Depicted in figure $3(a)$ is $\delta_{m} / \delta_{\omega, 0}$ for all runs given in table 1 . All layers show consistent growth with two plateaux corresponding to the two pairings, although TP500a5 and TP600a5 show fewer variations, owing to the larger $M L_{0}$ which makes the layers more difficult to entrain and thus impedes their growth. All cases have matched values of $\delta_{m} / \delta_{\omega, 0}$ from the beginning of the simulation until roll-up $\left(t^{*} \simeq 25\right)$, as the $R e_{0}$ or $M L_{0}$ specific characteristics are not apparent during roll-up, and then again for $t^{*}$ between 55 and 70 , which is the time between the first pairing $\left(40 \lesssim t^{*} \lesssim 50\right)$ and the second pairing $\left(75 \lesssim t^{*} \lesssim 90\right)$. In contrast, the layer growth is proportional to $M L_{0}$ between roll-up and the end of the first pairing as well as during the second pairing. All runs attain a local maximum for $t^{*}$ in the range 95 to 105 ; the transitional times given in table 1 correspond to these peaks in $\delta_{m} / \delta_{\omega, 0}$. The transitional-time value of $\delta_{m} / \delta_{\omega, 0}$ increases then decreases with $M L_{0}$ for both $R e_{0}$. This lack of monotonicity is a manifestation of the initial forcing which has a relatively weaker influence on the highest $M L_{0}$ layers. Note that the range of transitional $\delta_{m} / \delta_{\omega, 0}$ values is small (2.49 for SP600 to 2.73 for TP500a2, about 10\%).

The global mixing is measured by the product thickness $\delta_{P}$

$$
\delta_{P}=\int_{0}^{L_{3}} \int_{x_{2, \min }}^{x_{2, \max }} \int_{0}^{L_{1}} \rho\left[2 \min \left(Y_{V}, Y_{C}\right)\right] \mathrm{d} x_{1} \mathrm{~d} x_{2} \mathrm{~d} x_{3} .
$$

In our simulations, the low vapour mass fraction $\left(Y_{V} \lesssim 0.1\right)$ implies that $\delta_{P}$ is essentially twice the mass of vapour in the computational domain $\left(M_{V}\right)$. For the drop laden layers, $\delta_{P}$ is illustrated in figure $3(b)$, while $M_{V}$ is plotted in figure $3(c)$ (non-dimensionalized by the initial mass of gas, $M_{G, 0}$ ) The total liquid mass of the drops $\left(M_{L}\right)$, is plotted in figure $3(d)$ which is directly related to the ensemble-averaged drop diameter plotted in figure 3(e) (discussed below). Both $M_{V}$ and $M_{L}$ are seen to depend only on $M L_{0}$, with the plots for TP500a2 and TP600a2 $\left(M L_{0}=0.2\right)$ and those for TP500a5 and TP600a5 $\left(M L_{0}=0.5\right)$ overlapping, respectively. Understandably, $\delta_{P}$ increases with $M L_{0}$. The initial $\delta_{P}$ growth is larger for $M L_{0}=0.5$, but levels off sooner compared to $M L_{0}=0.2$, as vapour saturation intervenes in the lower stream and stops evaporation, similarly to the situation in Miller \& Bellan (1999); however, the ratio of $\delta_{P}$ between simulations with the same $R e_{0}$ is much less than that of $M L_{0}$ (e.g. 1.2 versus 2.5 at $t^{*}=105$ ). After a period of reduced increase rate, the $\delta_{P}$ growth for $M L_{0}=0.5$ then increases again, which is attributed to the increase in the ensemble-averaged drop temperature (see figure $3 f$ discussed below) that allows increased evaporation. By the end of the simulation, $\delta_{P}$ is still increasing for all layers, meaning that drops are still evaporating (the layer is not saturated). Notably, even at late times, much of the liquid has not evaporated and the portion of unevaporated liquid depends on $M L_{0}$ (e.g. $45 \%$ for $M L_{0}=0.2$ leading to $M L=0.09$, and $74 \%$ for $M L_{0}=0.5$ leading to $M L=0.037$ at $\left.t^{*}=105\right)$. This indicates that the interaction of mixing and drop evaporation is very complex, so that $M L_{0}$ is not quantitatively predictive of the final vapour mass.

Drop-based ensemble averages (denoted by $\langle\langle\rangle\rangle)$, here diameter-squared $\left(\left\langle\left\langle d^{2}\right\rangle\right\rangle\right)$ and temperature $\left(\left\langle\left\langle T_{d}\right\rangle\right\rangle\right)$, illustrated in figures $3 e$ and $3 f$, show a dependence only on 
$M L_{0}$, although $\left\langle\left\langle T_{d}\right\rangle\right\rangle$ differs slightly between the matched $M L_{0}$ cases for $80 \lesssim t^{*} \lesssim 120$. At the beginning of the simulations, $\left\langle\left\langle d^{2}\right\rangle\right\rangle$ decreases at the same rate for all cases, this being due to the same $T_{d, 0}$ and the same initial gas phase composition, but then the rate of decrease at the higher $M L_{0}$ quickly diminishes relative to the lower $M L_{0}$ cases, which is attributed to the accumulation of vapour in the gas phase (because $\left\langle\left\langle T_{d}\right\rangle\right\rangle$ is larger for $M L_{0}=0.5$, and therefore could not be the cause of decreased evaporation rate). Emulating $\left\langle\left\langle d^{2}\right\rangle\right\rangle$, the initial variation of $\left\langle\left\langle T_{d}\right\rangle\right\rangle$ is the same for all cases, in that it decreases due to the cooling effect of evaporation; but then, for $t^{*} \gtrsim 10$, an oscillatory behaviour of $\left\langle\left\langle T_{d}\right\rangle\right\rangle$ is observed, with the higher $M L_{0}$ cases having hotter drops since the reduced evaporation rate maintains a larger $\left\langle\left\langle T_{d}\right\rangle\right\rangle$. As elaborated by Le Clercq \& Bellan (2003), the later oscillations in $\left\langle\left\langle T_{d}\right\rangle\right\rangle$ are due to the competing effects of heating, which transfers energy from the gas phase, and evaporation, which releases energy into the surrounding gas.

Also depicted in figure 3 are two vorticity measures, the positive spanwise vorticity, $\left\langle\left\langle\omega_{3}^{+}\right\rangle\right\rangle$, and the enstrophy, $\left\langle\left\langle\omega_{i} \omega_{i}\right\rangle\right\rangle$, where $\langle\langle\rangle\rangle$ denotes averaging over all grid points. Initially null, $\omega_{3}^{+}$is a measure of the small-scale activity; $\omega_{i} \omega_{i}$ is related to stretching and tilting which is an important mechanism for turbulence production. Plotted in figure $3(g),\left\langle\left\langle\omega_{3}^{+}\right\rangle\right\rangle \delta_{\omega, 0} / \Delta U_{0}$ grows to an initial peak, at around the time of the first pairing, decreases and then grows again to a higher second peak, after which it declines. The time of the second peak is comparable for SP500 and SP600, being about $t^{*}=80$ and approximately corresponding to the time of the second pairing. For the TP cases, the peak is delayed, at about $t^{*}=95$, indicating that on a global basis the drops delay vorticity production. The maximum value of $\left\langle\left\langle\omega_{3}^{+}\right\rangle\right\rangle$increases with $M L_{0}$ at the same $R e_{0}$, indicating that the increasing number of drops causes increasing small-scale formation effects, and increases with $R e_{0}$ at the same $M L_{0}$, consistent with the well-known $R e_{0}$ effect for SP layers; TP600a5 has the largest peak value overall. Finally, $\left\langle\left\langle\omega_{i} \omega_{i}\right\rangle\right\rangle\left(\delta_{\omega, 0} / \Delta U_{0}\right)^{2}$ in figure $3(h)$ shows similar behaviour to $\left\langle\left\langle\omega_{3}^{+}\right\rangle\right\rangle \delta_{\omega, 0} / \Delta U_{0}$ in that it peaks sooner for the SP cases than for the TP cases, confirming the global indication that the drop presence delays the evolution of vorticity-related attributes, however, $\left\langle\left\langle\omega_{i} \omega_{i}\right\rangle\right\rangle\left(\delta_{\omega, 0} / \Delta U_{0}\right)^{2}$ peaks sooner than $\left\langle\left\langle\omega_{3}^{+}\right\rangle\right\rangle \delta_{\omega, 0} / \Delta U_{0}$. The TP600a2 curve has distinct behaviour compared to the other TP curves in that it exhibits more oscillations; however, TP600a5 reaches the maximum enstrophy whereas the other three TP curves attain similar maxima.

In looking at the four global measures depicted in figure 3, the results can be summarized as follows: (i) the variation of $\delta_{m} / \delta_{\omega, 0}$ depends on $M L_{0}$ and $R e_{0}$ but with no clear trend, (ii) $\delta_{P}$ increases with $M L_{0}$ but is insensitive to $R e_{0}$, and (iii) global small-scale generation and enstrophy production increase with $M L_{0}$ and with $R e_{0}$.

\subsection{Flow visualizations}

Flow visualizations are here scrutinized to detect characteristics that are unavailable in the global measures analysed above. To this end, contours of $\omega_{3} \delta_{\omega, 0} / \Delta U_{0}$ and the drop number density, $\rho_{n}$, for TP600a2 and TP600a5 are shown in figure 4, and contours of $Y_{V}$ and $T$ for the same cases are illustrated in figure 5. The vorticity is qualitatively similar for both cases, with significant numbers of small-scale structures distributed throughout the ultimate vortex comprising the mixing region. Notably, the increase in $M L_{0}$ decreases the local magnitude of the vorticity, presenting information unavailable from figure $3(g)$ which showed an increase in $\left\langle\left\langle\omega_{3}^{+}\right\rangle\right\rangle \delta_{\omega, 0} / \Delta U_{0}$ with $M L_{0}$. Although the ultimate vortex has been somewhat distorted by the small scales, it still retains some coherence at transition. The effect of the vorticity on the drop locations can be seen by comparing figures $4(a)$ and $4(b)$ with figures $4(c)$ and $4(d)$, 

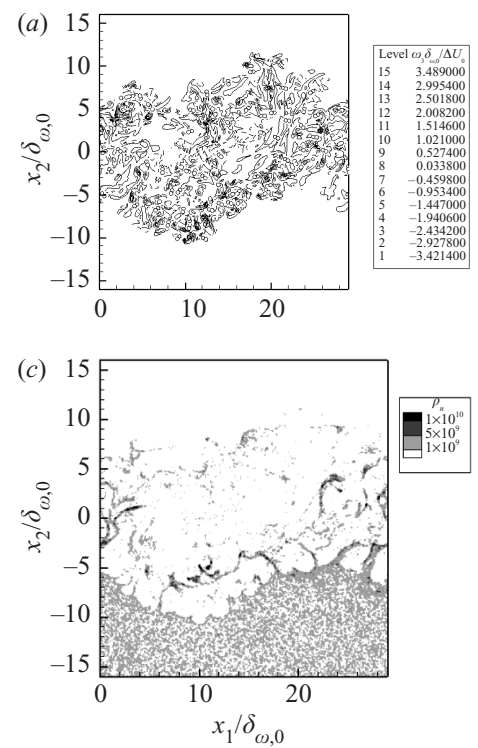
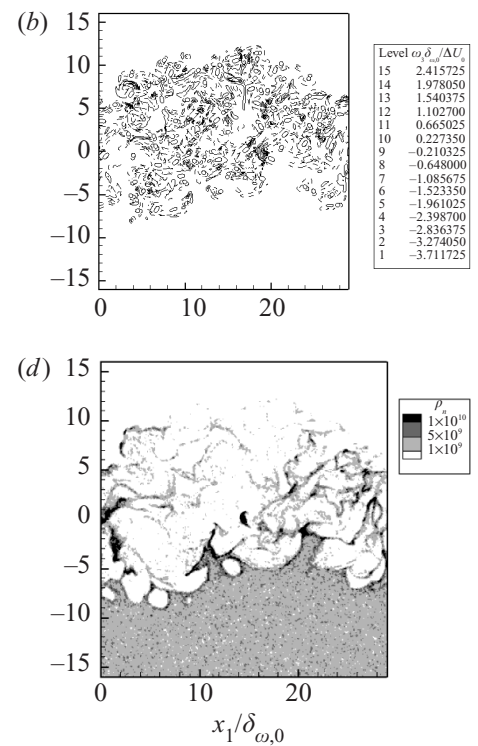

FIGURE 4. $(a, b)$ Spanwise vorticity and $(c, d)$ drop number density $\left(\mathrm{m}^{-3}\right)$ for $(a, c)$ TP600a2 at $t^{*}=105$ and $(b, d)$ TP600a5 at $t^{*}=105$, in the between-the-braid plane $\left(x_{3} / L_{3}=0.5\right)$. Dashed lines are used for negative values of vorticity.

respectively. The low $\rho_{n}$ regions within and on the lower stream side can be correlated with high vorticity magnitude regions; in contrast, the high $\rho_{n}$ regions occur at isolated regions within the layer and correlate with low vorticity, either in the free stream or on the edges of the ultimate vortex. The drop number density is generally smaller for TP600a2, mainly due to the effect of its smaller $M L_{0}$, but also due to the disappearance of a larger number of drops that have completely evaporated. An in-depth analysis of the drop organization conducted by Le Clercq \& Bellan (2003) and based on $\rho_{n}$ conditionally averaged on the second invariant of the deformation tensor for compressible flow, $I I_{u}=-\left[\left(S_{i j} S_{i j}-S_{k k} S_{l l}\right) / 2-\omega_{i} \omega_{i} / 4\right]$, revealed that at transition the drops accumulate mostly in regions where $I I_{u} \simeq 0$, then in regions where $I I_{u}<0$ and in the smallest number at locations where $I I_{u}>0$.

The gas phase temperature, displayed at transition for the TP600a2 and TP600a5 cases in figures $5(a)$ and $5(b)$, exhibits a lower stream where $T$ has decreased from its initial value due to heat transfer to the drops and is close to $T_{d, 0}$ (or $\left\langle\left\langle T_{d}\right\rangle\right\rangle$, see figure $3 f$ ), whereas the upper stream is still at $T_{C, 0}$. The mixing region is very inhomogeneous and is mostly composed of gas at an intermediate temperature of about $360 \mathrm{~K}$, with some colder gas within the layer and warmer layer gas found only adjacent to the warmer stream. The temperature range of TP600a5 (figure $5 b$ ) is slightly reduced compared to TP600a2 (figure $5 a$ ), and more of the colder gas has penetrated into the mixing region, consistent with the higher $M L_{0}$. The lowest $T$ regions correspond to locations of reduced pressure below the ultimate vortex, and therefore have a lower density than the adjacent lower-stream gas. The $Y_{V}$ contour plots displayed in figures $5(c)$ and $5(d)$ aid in the understanding of the $M L_{0}$-related behaviour of $\delta_{P}$ (figure $3 b$ ). Despite the much larger $M L_{0}$, the TP600a5 maximum $Y_{V}$ (about 0.10 ) is not substantially greater than that of TP600a2 (about 0.09). The gas within the vortex is an inhomogeneous mixture, with the largest $Y_{V}$ occurring either in the lower stream or at the periphery of the regions of high $\rho_{n}$, as shown 

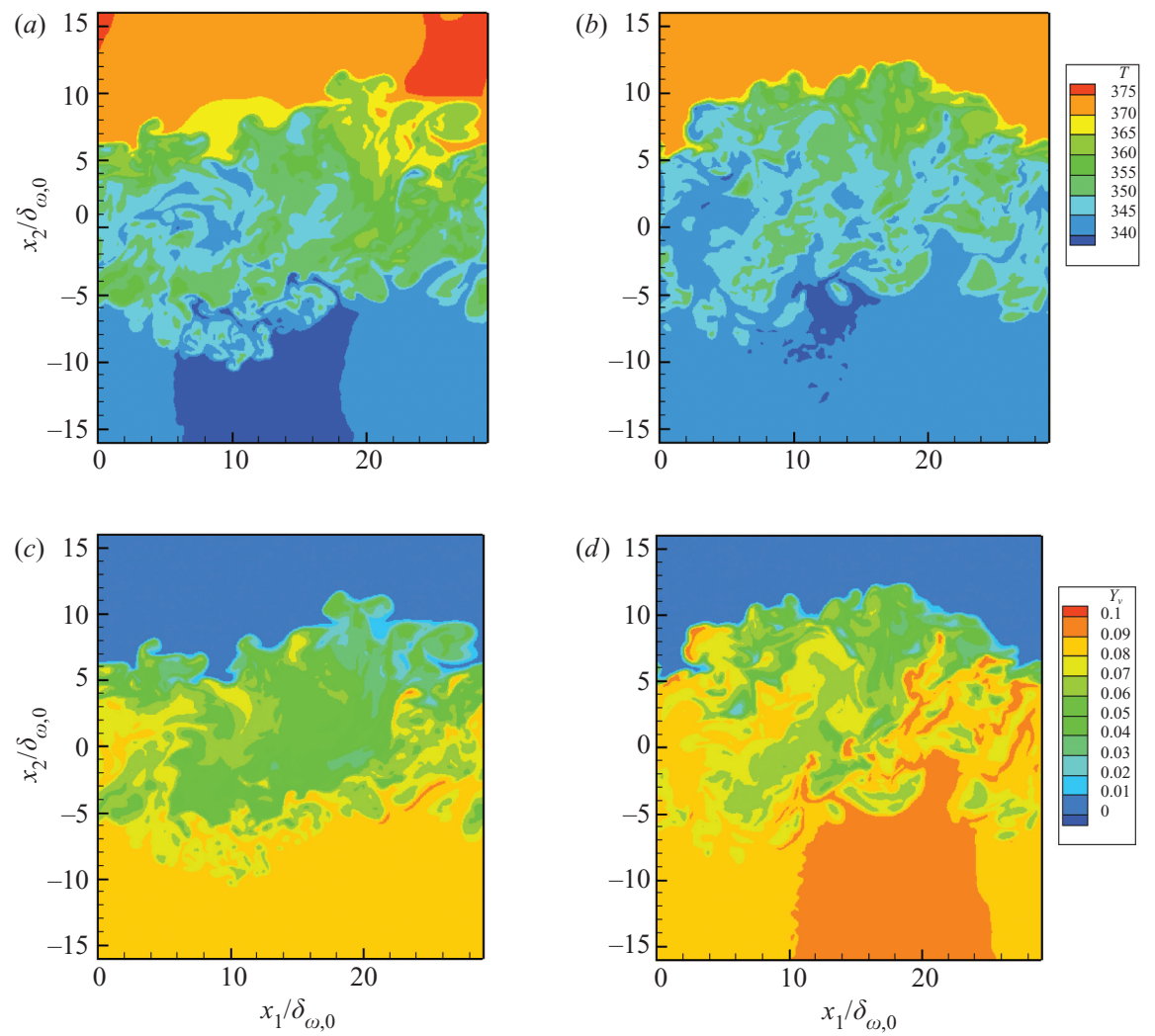

Figure 5. $(a, b)$ Temperature $(\mathrm{K})$ and $(c, d)$ vapour mass fraction for $(a, c)$ TP600a2 at $t^{*}=105$ and $(b, d)$ TP600a5 at $t^{*}=105$, in the between-the-braid plane.
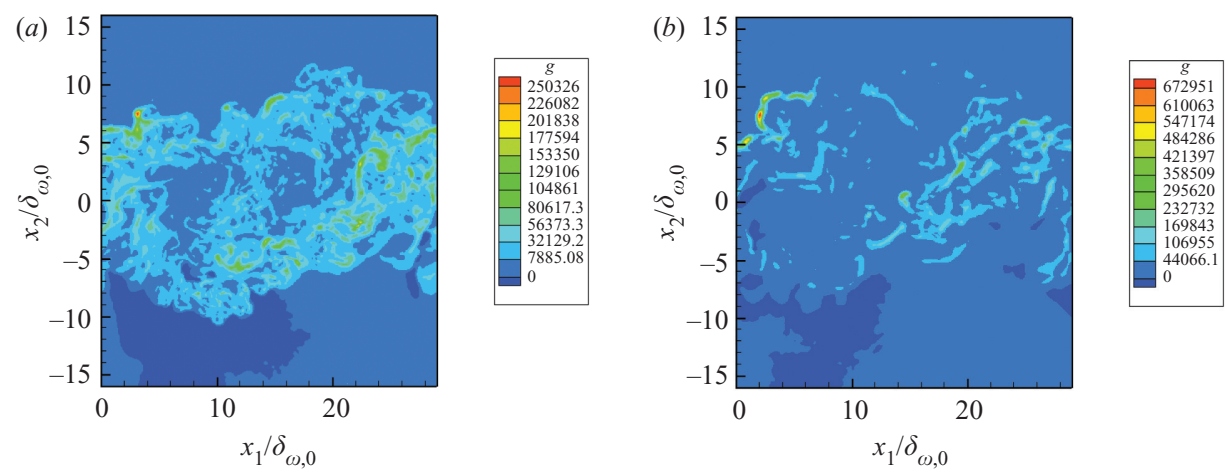

FIGURE 6. Irreversible entropy production $\left(\mathrm{Wm}^{-3} \mathrm{~K}^{-1}\right)$ for $(a)$ TP600a2 at $t^{*}=105$ and (b) TP600a5 at $t^{*}=105$, in the between-the-braid plane.

in figures $4(c)$ and $4(d)$. The highest $Y_{V}$ regions can be correlated with those of the high $\rho_{n}$ which occur at isolated locations within the layer. The explanation for this observation is that, although the entire layer is not saturated, by transition, the lower stream is close to the saturation $Y_{V}$ of about 0.09 . Therefore, continuing release of vapour can occur only within the mixing region, but of course only where the drops reside. 


$\begin{array}{lccc} & j=1 & j=2 & j=3 \\ j_{V j}(\text { r.m.s. } & 1.4196 \times 10^{-2} & 1.5865 \times 10^{-2} & 1.6586 \times 10^{-2} \\ j_{V j}\left(Y_{V}\right) \text { (r.m.s.) } & 1.4001 \times 10^{-2} & 1.5718 \times 10^{-2} & 1.6478 \times 10^{-2} \\ j_{V j}(p) \text { (r.m.s.) } & 3.0602 \times 10^{-3} & 2.9603 \times 10^{-3} & 2.8889 \times 10^{-3} \\ j_{V j}\left(Y_{V}\right) / j_{V j} \text { (LS) } & 0.9631 & 0.9733 & 0.9784 \\ j_{V j}(p) / j_{V j} \text { (LS) } & 0.0369 & 0.0267 & 0.0216\end{array}$

r.m.s. $=$ root-mean-square, $\mathrm{LS}=$ least-squares fit slope

TABLE 2. Vapour mass flux $\left(\mathrm{kg} \mathrm{m}^{-2} \mathrm{~s}^{-1}\right)$, TP600a5, $t^{*}=105$.

$\begin{array}{lccc} & j=1 & j=2 & j=3 \\ q_{j} \text { (r.m.s.) } & 8.3640 \times 10^{3} & 9.3573 \times 10^{3} & 9.7093 \times 10^{3} \\ -\lambda \frac{\partial T}{\partial x_{j}} \text { (r.m.s.) } & 4.4579 \times 10^{3} & 4.9651 \times 10^{3} & 5.1896 \times 10^{3} \\ \left(h_{V}-h_{C}\right) j_{V j} \text { (r.m.s.) } & 1.2303 \times 10^{4} & 1.3750 \times 10^{4} & 1.4381 \times 10^{4} \\ -\lambda \frac{\partial T}{\partial x_{j}} / q_{j}(\mathrm{LS}) & -0.4398 & -0.4388 & -0.4541 \\ \left(h_{V}-h_{C}\right) j_{V j} / q_{j}(\mathrm{LS}) & 1.4398 & 1.4388 & 1.4541\end{array}$

r.m.s. $=$ root-mean-square, $\mathrm{LS}=$ least-squares fit slope

TABLE 3. Heat flux $\left(\mathrm{J} \mathrm{m}^{-2} \mathrm{~s}^{-1}\right)$, TP600a5, $t^{*}=105$.

\subsection{Species-mass and heat flux budgets}

The presence of both $T$ and $Y_{V}$ gradients in TP flows with evaporating drops directs attention to the form of the vapour mass flux (2.13) and of the heat flux (2.15). For ease of analysis, each flux is split into two terms

$$
\begin{gathered}
j_{V j}=j_{V j}\left(Y_{V}\right)+j_{V j}(p), \quad q_{j}=q_{j}(T)+q_{j}(h), \\
j_{V j}\left(Y_{V}\right)=-\rho D \frac{\partial Y_{V}}{\partial x_{j}}, \quad j_{V j}(p)=-Y_{C} Y_{V}\left(Y_{V}+\frac{Y_{C} m_{V}}{m_{C}}\right)\left[\frac{m_{C}}{m_{V}}-1\right] \frac{\rho D}{p} \frac{\partial p}{\partial x_{j}}, \\
q_{j}(T)=-\lambda \frac{\partial T}{\partial x_{j}}, \quad q_{j}(h)=\left(h_{V}-h_{C}\right) j_{V j} .
\end{gathered}
$$

To evaluate the importance of terms $j_{V j}(p)$ and $q_{j}(h)$ with respect to $j_{V j}\left(Y_{V}\right)$ and $q_{j}(T)$, respectively, the DNS database of the TP cases was analysed at their transitional state; the results for TP600a5 are given in table 2 for $j_{V j}$ and in table 3 for $q_{j}$. Comparisons of two quantities defined at all grid points, $\mathscr{X}\left(x_{1}, x_{2}, x_{3}\right)$ and $\mathscr{Y}\left(x_{1}, x_{2}, x_{3}\right)$, are carried out using a least-squares (LS) fit of $\mathscr{Y}=b \mathscr{X}$ which leads to the slope

and the correlation

$$
b=\frac{\langle\langle\mathscr{X} \mathscr{Y}\rangle\rangle}{\langle\langle\mathscr{X} \mathscr{X}\rangle\rangle}
$$

$$
\mathscr{R}(\mathscr{X}, \mathscr{Y})=\frac{\langle\langle\mathscr{X} \mathscr{Y}\rangle\rangle-\langle\langle\mathscr{X}\rangle\rangle\langle\langle\mathscr{Y}\rangle\rangle}{\sqrt{\left(\left\langle\left\langle\mathscr{X}^{2}\right\rangle\right\rangle-\langle\langle\mathscr{X}\rangle\rangle^{2}\right)} \sqrt{\left(\left\langle\left\langle\mathscr{Y}^{2}\right\rangle\right\rangle-\langle\langle\mathscr{Y}\rangle\rangle^{2}\right)}},
$$

where $\mathscr{R}^{2}$ is the fraction of the variation in $\mathscr{Y}$ due to the variation of $\mathscr{X}$ (Ayyub \& McCuen 1997). From the LS slope comparison of $j_{V j}(p)$ and $j_{V j}\left(Y_{V}\right)$ with $j_{V j}$, it is obvious that the $j_{V j}(p)$ contribution is negligible and that more than $96 \%$ is due to 
$j_{V j}\left(Y_{V}\right)$. Clearly, in all directions, the root-mean-square (r.m.s.) magnitude of $j_{V j}$ is close to that of $j_{V j}\left(Y_{V}\right)$ and the $j_{V j}(p)$ r.m.s. is about one-fifth of that of $j_{V j}\left(Y_{V}\right)$. Therefore, it seems legitimate to neglect the pressure contribution in $j_{V j}$. In contrast, the dominant contribution to $q_{j}$ comes from $q_{j}(h)$, whose LS slope is three times the magnitude of $q_{j}(T)$ and, moreover, is of different sign, indicating that the enthalpy term should be included in the energy equation. Neglecting $q_{j}(h)$ would lead to the heat transfer vector having an opposite orientation. Also, the r.m.s. of $q_{j}(h)$ is larger than that of $q_{j}(T)$, reinforcing the conclusion that $q_{j}(h)$ must be included in $q_{j}$.

\section{Derivation of gas-phase LES equations}

Following the SP protocol, the LES equations are here derived by spatially filtering the gas-phase DNS equations described in $\S 2.1$, resulting in various unclosed terms that cannot be computed from the filtered flow field. Next, certain simplifying assumptions are assessed that could reduce the complexity of the original LES equations. Finally, the budget of the LES equations is computed to determine if the LES equations can be further simplified by neglecting certain terms. This process identifies those unclosed terms that will require explicit modelling.

The LES uses a mathematical description similar to that of DNS, i.e. Eulerian for the gas phase and Lagrangian for the liquid phase. Drops tracked in the LES will follow the DNS evolution equations presented in $\S 2.2$; however, the LES may use computational drops (defined in $\S 6.2$ ) rather than physical drops.

\subsection{Filtered gas-phase DNS equations}

The filtering operation is defined as:

$$
\bar{\psi}(\boldsymbol{x})=\int_{V_{f}} \psi(\boldsymbol{y}) G(\boldsymbol{x}-\boldsymbol{y}) \mathrm{d} \boldsymbol{y},
$$

where $V_{f}$ is the filtering volume and $G$ is the filter function, with the property that if $\psi$ is spatially uniform, then $\bar{\psi}=\psi$. For finite-difference computations, the filter of choice is a top-hat filter, which is adopted here; then $\bar{\psi}$ is simply the volume-average. The Favre (density-weighted) filtering is defined as $\tilde{\psi}=\overline{\rho \psi} / \bar{\rho}$. It is also assumed that filtering and differentiation commute, which is correct except near boundaries (because the size of the filtering volume decreases as the boundary is approached). The filtered gas-phase equations are

$$
\begin{gathered}
\frac{\partial \bar{\rho}}{\partial t}+\frac{\partial\left(\bar{\rho} \tilde{u}_{j}\right)}{\partial x_{j}}=\bar{S}_{I}, \\
\frac{\partial\left(\bar{\rho} \tilde{u}_{i}\right)}{\partial t}+\frac{\partial\left(\overline{\rho u_{i} u_{j}}\right)}{\partial x_{j}}=-\frac{\partial \bar{p}}{\partial x_{i}}+\frac{\partial \bar{\sigma}_{i j}}{\partial x_{j}}+\bar{S}_{I I, i}, \\
\frac{\partial\left(\bar{\rho} \tilde{e}_{t}\right)}{\partial t}+\frac{\partial\left(\overline{\rho e_{t} u_{j}}\right)}{\partial x_{j}}=-\frac{\partial\left(\overline{p u_{j}}\right)}{\partial x_{j}}-\frac{\partial \bar{q}_{j}}{\partial x_{j}}+\frac{\partial\left(\bar{\sigma}_{i j} u_{i}\right)}{\partial x_{j}}+\bar{S}_{I I I}, \\
\frac{\partial\left(\bar{\rho} \tilde{Y}_{V}\right)}{\partial t}+\frac{\partial\left(\overline{\rho Y_{V} u_{j}}\right)}{\partial x_{j}}=-\frac{\partial \bar{j}_{V j}}{\partial x_{j}}+\bar{S}_{I} .
\end{gathered}
$$

From (4.1), the FSTs $\left(\bar{S}=\left\{\bar{S}_{I}, \bar{S}_{I I, i}, \bar{S}_{I I I}\right\}\right)$ are properly interpreted by considering a drop located at $\boldsymbol{X}$ within the filtering volume $V_{f}$ and its contribution within that volume

$$
\bar{S}(\boldsymbol{x})=\int_{V_{f}} S_{d} \delta(\boldsymbol{y}-\boldsymbol{X}) G(\boldsymbol{x}-\boldsymbol{y}) \mathrm{d} \boldsymbol{y},
$$


where $S_{d} \delta(\boldsymbol{y}-\boldsymbol{X})$ is the point-source contribution from the drop and $\delta$ is the delta function. When $G$ is a top-hat filter, the FST is

$$
\bar{S}\left(\psi_{f}, Z\right)=\frac{1}{V_{f}} \sum_{\beta}\left[S_{d}\left(\psi_{f}, Z\right)\right]_{\beta},
$$

a volume-average over the drops $\beta$ within the filtering volume, where $S_{d}$ was defined in (2.27). (Note that $\bar{S}$ is not obtained by filtering the DNS $S$ obtained through (2.31), because $S$ depends on the grid spacing whereas $\bar{S}$ should depend only on the filter width.)

Using the adopted notation of denoting the DNS flow field as $\phi$, the filtered flow field can now be denoted as $\bar{\phi}$ and we can formally define a function $f(\bar{\phi})$ that has the same functional form as in the DNS but is in general different from its filtered counterpart $\overline{f(\phi)}$. For example, $\tilde{e}=\overline{\rho e(\phi)} / \bar{\rho}=\tilde{e}_{t}-\overline{u_{i} u_{i}} / 2$, whereas $e(\bar{\phi})=\tilde{e}_{t}-\tilde{u}_{i} \tilde{u}_{i} / 2$. Moreover, we define SGS fluxes through

$$
\tau_{i j}=\widetilde{u_{i} u_{j}}-\tilde{u}_{i} \tilde{u}_{j}, \quad \zeta_{j}=\widetilde{h u_{j}}-\tilde{h} \tilde{u}_{j}, \quad \eta_{j}=\widetilde{Y_{V} u_{j}}-\tilde{Y}_{V} \tilde{u}_{j}
$$

where $\tilde{h}=\tilde{e}+\bar{p} / \bar{\rho}$. With these notations, the filtered equations become

$$
\begin{gathered}
\frac{\partial \bar{\rho}}{\partial t}+\frac{\partial\left(\bar{\rho} \tilde{u}_{j}\right)}{\partial x_{j}}=\bar{S}_{I}, \\
\frac{\partial\left(\bar{\rho} \tilde{u}_{i}\right)}{\partial t}+\frac{\partial\left(\bar{\rho} \tilde{u}_{i} \tilde{u}_{j}\right)}{\partial x_{j}}=-\frac{\partial[p(\bar{\phi})]}{\partial x_{i}}+\frac{\partial \sigma_{i j}(\bar{\phi})}{\partial x_{j}}+\bar{S}_{I I, i}-\frac{\partial\left(\bar{\rho} \tau_{i j}\right)}{\partial x_{j}} \\
\quad-\frac{\partial}{\partial x_{i}}[\bar{p}-p(\bar{\phi})]+\frac{\partial}{\partial x_{j}}\left[\bar{\sigma}_{i j}-\sigma_{i j}(\bar{\phi})\right] \\
\frac{\partial\left(\bar{\rho} \tilde{e}_{t}\right)}{\partial t}+\frac{\partial\left(\bar{\rho} \tilde{e}_{t} \tilde{u}_{j}\right)}{\partial x_{j}}=-\frac{\partial\left[p(\bar{\phi}) \tilde{u}_{j}\right]}{\partial x_{j}}-\frac{\partial q_{j}(\bar{\phi})}{\partial x_{j}}+\frac{\partial\left[\sigma_{i j}(\bar{\phi}) \tilde{u}_{i}\right]}{\partial x_{j}}+\bar{S}_{I I I}-\frac{\partial\left(\bar{\rho} \zeta_{j}\right)}{\partial x_{j}} \\
-\frac{\partial}{\partial x_{j}}\left[\frac{1}{2}\left(\bar{\rho} u_{i} u_{i} u_{j}-\bar{\rho} \bar{q}_{j} u_{i} \tilde{u}_{j}\right)\right]-\frac{\partial}{\partial x_{j}}\left\{[\bar{p}-p(\bar{\phi})] \tilde{u}_{j}\right\} \\
\frac{\partial\left(\bar{\rho} \tilde{Y}_{V}\right)}{\partial t}+\frac{\partial\left(\bar{\rho} \tilde{Y}_{V} \tilde{u}_{j}\right)}{\partial x_{j}}=-\frac{\partial j_{V j}(\bar{\phi})}{\partial x_{j}}+\bar{S}_{I}-\frac{\partial\left(\bar{\rho} \eta_{j}\right)}{\partial x_{j}}-\frac{\partial}{\partial x_{j}}\left[\bar{j}_{V j}-j_{V j}(\bar{\phi}) \tilde{u}_{i}\right],
\end{gathered}
$$

Equations (4.9)-(4.12) for $\bar{\phi}$ contain terms that cannot be computed directly from $\bar{\phi}$ and that therefore must be modelled, namely, (i) the SGS fluxes $\left(\tau_{i j}, \zeta_{j}, \eta_{j}\right)$, (ii) the FSTs $\left(\bar{S}_{I}, \bar{S}_{I I, i}, \bar{S}_{I I I}\right)$, (iii) the velocity triple correlation $\left(\overline{\rho u_{i} u_{i} u_{j}}-\overline{\rho u_{i} u_{i}} \tilde{u}_{j}\right)$ and (iv) the difference terms $\left(\bar{p}-p(\bar{\phi}), \bar{\sigma}_{i j}-\sigma_{i j}(\bar{\phi}), \bar{q}_{j}-q_{j}(\bar{\phi}), \bar{\sigma}_{i j} u_{i}-\sigma_{i j}(\bar{\phi}) \tilde{u}_{i}, \bar{j}_{V j}-j_{V j}(\bar{\phi})\right)$.

\subsection{LES assumptions}

To reduce the modelling requirements, the $\overline{f(\phi)}=f(\bar{\phi})$ assumption is evaluated a priori : for the thermodynamic quantities,

$$
\tilde{e}=e(\bar{\phi}), \quad \bar{T}=T(\bar{\phi}), \quad \widetilde{T}=T(\bar{\phi}), \quad \bar{p}=p(\bar{\phi}), \quad \tilde{h}=h(\bar{\phi}) ;
$$

for the viscous stresses and the velocity-viscous stress correlation,

$$
\sigma_{i j}=\sigma_{i j}(\bar{\phi}), \quad \overline{u_{i} \sigma_{i j}}=\tilde{u}_{i} \sigma_{i j}(\bar{\phi}) ;
$$

and for the heat and mass fluxes,

$$
\bar{j}_{V j}=j_{V j}(\bar{\phi}), \quad \bar{q}_{j}=q_{j}(\bar{\phi}) .
$$


Fluxes

\begin{tabular}{ll}
\hline Exact & Model \\
$\bar{J}_{V 1}$ & $J_{V 1}(\bar{\phi})$ \\
$\bar{J}_{V 2}$ & $J_{V 2}(\bar{\phi})$ \\
$\bar{J}_{V 3}$ & $J_{V 3}(\bar{\phi})$ \\
$\bar{q}_{1}$ & $q_{1}(\bar{\phi})$ \\
$\bar{q}_{2}$ & $q_{2}(\bar{\phi})$ \\
$\bar{q}_{3}$ & $q_{3}(\bar{\phi})$
\end{tabular}

\begin{tabular}{cccccc}
\multicolumn{6}{c}{ Slopes } \\
\hline SP500 & TP500a2 & TP500a5 & SP600 & TP600a2 & TP600a5 \\
- & 0.9872 & 0.9804 & - & 0.9905 & 0.9847 \\
- & 0.9853 & 0.9826 & - & 0.9892 & 0.9850 \\
- & 0.9888 & 0.9840 & - & 0.9917 & 0.9860 \\
1.0293 & 0.9989 & 0.9962 & 1.0381 & 1.0008 & 0.9982 \\
1.1235 & 0.9987 & 0.9965 & 1.0820 & 1.0004 & 0.9974 \\
1.1077 & 1.0031 & 1.0003 & 1.0816 & 1.0046 & 1.0013 \\
\multicolumn{5}{c}{ Slope $=\frac{\text { Model }}{\text { Exact }}$ from least squares fit } \\
\end{tabular}

TABLE 4. LES assumptions for heat and mass fluxes at transitional times, $\bar{\Delta}=8 \Delta x$.

For the velocity triple correlation, we assume (see Okong'o, Knight \& Zhou 2000)

$$
\frac{1}{2}\left(\overline{\rho u_{i} u_{i} u_{j}}-\overline{\rho u_{i} u_{i}} \tilde{u}_{j}\right)=\bar{\rho} \tau_{i j} \tilde{u}_{i} .
$$

The evaluation of the LES assumptions displayed in (4.13)-(4.16) was performed on the filtered DNS databases at the transitional $t^{*}$. For the cubic top-hat filter used here, the filtering volume has sides of length $\bar{\Delta}$. The present results use $\bar{\Delta}=4 \Delta x$ and $\bar{\Delta}=8 \Delta x$, where $\Delta x=\max \left(\Delta x_{1}, \Delta x_{2}, \Delta x_{3}\right)$ (the grid spacing in the three coordinate directions is approximately equal; see table 1). For each of (4.13)-(4.16), the model (right-hand side of the equations) is linearly correlated to the exact quantity (lefthand side) using a least-squares fit. If the assumptions were exactly correct, both the slope and the correlation (computed from (3.6) and (3.7)) of the model to the exact quantity would be unity. Calculations on all databases yielded slopes of 1.003 to 1.010 and correlations of 0.9931 to 1.000 for (4.13), and slopes of 0.9997 to 1.0005 and correlations of 0.9948 to 1.000 for the viscous stresses $\left(\bar{\sigma}_{i j}=\sigma_{i j}(\bar{\phi})\right)$. The velocitystress correlation $\left(\overline{u_{i} \sigma_{i j}}=\tilde{u}_{i} \sigma_{i j}(\bar{\phi})\right)$, the mass and heat fluxes (4.15), and the velocity triple correlation (4.16) had slopes and correlations almost as close to unity, ranging from 0.985 to 1.124 and 0.945 to 0.999 , respectively. Correlations and slopes generally had a larger deviation from unity at the larger filter width. Table 4 , for $\bar{\Delta}=8 \Delta x$, shows that the assumption for the heat flux (that $\bar{q}_{j}=q_{j}(\phi)$ ) appears to hold even better for the TP than for the SP cases; this tendency was less evident for $\bar{\Delta}=4 \Delta x$.

Therefore, the examination of the LES assumptions (4.13)-(4.16) shows that they are justified, and that the appropriate simplifications could be made in (4.10)-(4.12). However, (4.10)-(4.12) contain derivatives of the quantities whose near equality was evaluated, and these derivatives are more sensitive to modelling errors than the undifferentiated quantities, prompting us to further consider the budgets of these equations.

\subsection{Budgets of the filtered equations}

The budgets of (4.10)-(4.12) are displayed in tables 5-9. Results are listed in the tables for the filtered flow fields of the $R e_{0}=600$ cases at the transitional $t^{*}$ indicated, with $\bar{\Delta}=4 \Delta x$ and $\bar{\Delta}=8 \Delta x$ (results for $R e_{0}=500$ were analysed but are not shown). All computations are performed using all grid points, i.e. on the DNS (fine) grid, and therefore do not include the discretization error incurred when the differentiations are performed on a presumably coarser LES grid. Although the flow evolution will not be the same in the LES as in the DNS, it seems reasonable to suppose that the unclosed terms of larger magnitude would need to be modelled more accurately. The 


\begin{tabular}{|c|c|c|c|c|c|c|}
\hline \multirow[b]{2}{*}{$x_{1}$ Momentum } & \multicolumn{3}{|c|}{$\bar{\Delta}=4 \Delta x$} & \multicolumn{3}{|c|}{$\bar{\Delta}=8 \Delta x$} \\
\hline & SP600 & ТР600a2 & TP600a5 & SP600 & ТP600a2 & ТР600a5 \\
\hline$t^{*}$ & 100 & 105 & 105 & 100 & 105 & 105 \\
\hline$\frac{\partial}{\partial x_{j}}\left(\bar{\rho} \tilde{u}_{1} \tilde{u}_{j}\right)$ & $3.0189 \times 10^{5}$ & $3.5057 \times 10^{5}$ & $3.7229 \times 10^{5}$ & $2.3156 \times 10^{5}$ & $2.5084 \times 10^{5}$ & $2.5697 \times 10^{5}$ \\
\hline$\frac{\partial}{\partial x_{1}}[p(\bar{\phi})]$ & $1.8383 \times 10^{5}$ & $2.0398 \times 10^{5}$ & $2.0457 \times 10^{5}$ & $1.6600 \times 10^{5}$ & $1.6780 \times 10^{5}$ & $1.6006 \times 10^{5}$ \\
\hline$\frac{\partial}{\partial x_{j}}\left[\sigma_{1 j}(\bar{\phi})\right]$ & $9.6983 \times 10^{3}$ & $1.1822 \times 10^{4}$ & $1.2642 \times 10^{4}$ & $4.8083 \times 10^{3}$ & $5.6936 \times 10^{3}$ & $6.1080 \times 10^{3}$ \\
\hline $\bar{S}_{I I, 1}$ & 0 & $1.0295 \times 10^{4}$ & $2.7318 \times 10^{4}$ & 0 & $7.6582 \times 10^{3}$ & $2.0126 \times 10^{4}$ \\
\hline$\frac{\partial}{\partial x_{j}}\left(\bar{\rho} \tau_{1 j}\right)$ & $3.1105 \times 10^{4}$ & $4.8058 \times 10^{4}$ & $5.1557 \times 10^{4}$ & $3.8166 \times 10^{4}$ & $5.5275 \times 10^{4}$ & $5.9256 \times 10^{4}$ \\
\hline$\frac{\partial}{\partial x_{1}}[\Delta(\bar{p})]$ & $1.4540 \times 10^{4}$ & $2.0341 \times 10^{4}$ & $2.2002 \times 10^{4}$ & $1.5676 \times 10^{4}$ & $1.9969 \times 10^{4}$ & $2.1764 \times 10^{4}$ \\
\hline$\frac{\partial}{\partial x_{j}}\left[\Delta\left(\bar{\sigma}_{1 j}\right)\right]$ & $7.8458 \times 10^{1}$ & $\begin{array}{r}2.6273 \times 10^{2} \\
\Delta(\bar{f}) \equiv\end{array}$ & $2.9290 \times 10^{2}$ & $2.4730 \times 10^{2}$ & $2.8344 \times 10^{2}$ & $3.9732 \times 10^{2}$ \\
\hline
\end{tabular}

TABLE 5. Magnitude (r.m.s.) of terms in LES equations at transitional times. Units are $\mathrm{N} \mathrm{m}^{-3}$.

\begin{tabular}{cccccccc}
\hline & \multicolumn{3}{c}{$\bar{\Delta}=4 \Delta x$} & & \multicolumn{3}{c}{$\bar{\Delta}=8 \Delta x$} \\
\cline { 2 - 4 } \cline { 7 - 8 }$x_{2}$ Momentum & SP600 & TP600a2 & TP600a5 & & SP600 & TP600a2 & TP600a5 \\
$t^{*}$ & 100 & 105 & 105 & & 100 & 105 & 105 \\
$\frac{\partial}{\partial x_{j}}\left(\bar{\rho} \tilde{u}_{2} \tilde{u}_{j}\right)$ & $3.3349 \times 10^{5}$ & $4.1267 \times 10^{5}$ & $4.4661 \times 10^{5}$ & & $2.3643 \times 10^{5}$ & $2.7925 \times 10^{5}$ & $3.0365 \times 10^{5}$ \\
$\frac{\partial}{\partial x_{2}}[p(\bar{\phi})]$ & $1.3690 \times 10^{5}$ & $1.8192 \times 10^{5}$ & $1.8367 \times 10^{5}$ & & $1.1166 \times 10^{5}$ & $1.3544 \times 10^{5}$ & $1.3193 \times 10^{5}$ \\
$\frac{\partial}{\partial x_{j}}\left[\sigma_{2 j}(\bar{\phi})\right]$ & $8.5216 \times 10^{3}$ & $1.0611 \times 10^{4}$ & $1.1105 \times 10^{4}$ & & $4.7701 \times 10^{3}$ & $5.2538 \times 10^{3}$ & $5.5265 \times 10^{3}$ \\
$\bar{S}_{I I, 2}$ & 0 & $6.9249 \times 10^{3}$ & $2.0353 \times 10^{4}$ & 0 & $4.8362 \times 10^{3}$ & $1.4361 \times 10^{4}$ \\
$\frac{\partial}{\partial x_{j}}\left(\bar{\rho} \tau_{2 j}\right)$ & $3.0800 \times 10^{4}$ & $4.8879 \times 10^{4}$ & $5.2282 \times 10^{4}$ & & $3.7772 \times 10^{4}$ & $5.6863 \times 10^{4}$ & $5.9078 \times 10^{4}$ \\
$\frac{\partial}{\partial x_{2}}[\Delta(\bar{p})]$ & $1.7619 \times 10^{4}$ & $2.3147 \times 10^{4}$ & $2.5343 \times 10^{4}$ & & $1.9173 \times 10^{4}$ & $2.3912 \times 10^{4}$ & $2.5836 \times 10^{4}$ \\
$\frac{\partial}{\partial x_{j}}\left[\Delta\left(\bar{\sigma}_{2 j}\right)\right]$ & $6.5071 \times 10^{2}$ & $4.4696 \times 10^{2}$ & $5.4342 \times 10^{2}$ & & $2.3905 \times 10^{3}$ & $1.4599 \times 10^{3}$ & $1.8523 \times 10^{3}$
\end{tabular}

TABLE 6. Magnitude (r.m.s.) of terms in LES equations at transitional times. Units are $\mathrm{N} \mathrm{m}^{-3}$.

studied flow fields are relevant for LES since a significant portion of the domain turbulent kinetic energy (TKE) resides in the SGS, ranging from $7 \%$ to $29 \%$ for the six cases in the database. For each case, the portion of the domain TKE in the SGS is about twice as large at $\bar{\Delta}=8 \Delta x$ than at $\bar{\Delta}=4 \Delta x$. At a given $\bar{\Delta}$, the TKE portion residing in the SGS is smaller at the larger $R e_{0}$ and tends to increase with increasing $M L_{0}$.

For all equations, terms can be grouped as largest, intermediate and smallest, with the relative magnitude of terms possibly varying within each group depending on $R e_{0}, M L_{0}$ and $\bar{\Delta}$. For the momentum equations (tables 5-7), the largest contributions 


\begin{tabular}{cccccccc}
\hline & \multicolumn{3}{c}{$\bar{\Delta}=4 \Delta x$} & & \multicolumn{3}{c}{$\bar{\Delta}=8 \Delta x$} \\
\cline { 3 - 4 }$x_{3}$ Momentum & SP600 & TP600a2 & TP600a5 & & SP600 & TP600a2 & TP600a5 \\
$t^{*}$ & 100 & 105 & 105 & & 100 & 105 & 105 \\
$\frac{\partial}{\partial x_{j}}\left(\bar{\rho} \tilde{u}_{3} \tilde{u}_{j}\right)$ & $3.1457 \times 10^{5}$ & $4.0645 \times 10^{5}$ & $4.3790 \times 10^{5}$ & & $1.9954 \times 10^{5}$ & $2.5747 \times 10^{5}$ & $2.8035 \times 10^{5}$ \\
$\frac{\partial}{\partial x_{3}}[p(\bar{\phi})]$ & $1.0530 \times 10^{5}$ & $1.5672 \times 10^{5}$ & $1.7119 \times 10^{5}$ & & $7.0193 \times 10^{4}$ & $1.0147 \times 10^{5}$ & $1.1331 \times 10^{5}$ \\
$\frac{\partial}{\partial x_{j}}\left[\sigma_{3 j}(\bar{\phi})\right]$ & $7.5605 \times 10^{3}$ & $9.2435 \times 10^{3}$ & $9.9855 \times 10^{3}$ & & $3.6196 \times 10^{3}$ & $4.3543 \times 10^{3}$ & $4.6140 \times 10^{3}$ \\
$\bar{S}_{I I, 3}$ & 0 & $5.5845 \times 10^{3}$ & $1.7995 \times 10^{4}$ & 0 & $3.5326 \times 10^{3}$ & $1.2056 \times 10^{4}$ \\
$\frac{\partial}{\partial x_{j}}\left(\bar{\rho} \tau_{3 j}\right)$ & $3.1263 \times 10^{4}$ & $4.8471 \times 10^{4}$ & $5.3294 \times 10^{4}$ & & $3.8569 \times 10^{4}$ & $5.6031 \times 10^{4}$ & $6.0466 \times 10^{4}$ \\
$\frac{\partial}{\partial x_{3}}[\Delta(\bar{p})]$ & $1.8425 \times 10^{4}$ & $2.4512 \times 10^{4}$ & $2.6172 \times 10^{4}$ & & $2.0470 \times 10^{4}$ & $2.5489 \times 10^{4}$ & $2.7479 \times 10^{4}$ \\
$\frac{\partial}{\partial x_{j}}\left[\Delta\left(\bar{\sigma}_{3 j}\right)\right]$ & $1.2952 \times 10^{3}$ & $7.9873 \times 10^{2}$ & $1.0111 \times 10^{3}$ & & $4.7803 \times 10^{3}$ & $2.9092 \times 10^{3}$ & $3.6961 \times 10^{3}$
\end{tabular}

TABLE 7. Magnitude (r.m.s.) of terms in LES equations at transitional times. Units are $\mathrm{N} \mathrm{m}^{-3}$.

\begin{tabular}{|c|c|c|c|c|c|c|}
\hline \multirow[b]{2}{*}{ Energy } & \multicolumn{3}{|c|}{$\bar{\Delta}=4 \Delta x$} & \multicolumn{3}{|c|}{$\bar{\Delta}=8 \Delta x$} \\
\hline & SP600 & ТP600a2 & ТР600a5 & SP600 & TP600a2 & TP600a 5 \\
\hline$t^{*}$ & 100 & 105 & 105 & 100 & 105 & 105 \\
\hline$\frac{\partial}{\partial x_{j}}\left(\bar{\rho} \tilde{\boldsymbol{e}}_{t} \tilde{u}_{j}\right)$ & $4.4946 \times 10^{7}$ & $2.2398 \times 10^{8}$ & $2.7730 \times 10^{8}$ & $7.2909 \times 10^{7}$ & $1.5981 \times 10^{8}$ & $1.9684 \times 10^{8}$ \\
\hline$\frac{\partial}{\partial x_{j}}\left[p(\bar{\phi}) \tilde{u}_{j}\right]$ & $1.5226 \times 10^{7}$ & $1.7014 \times 10^{7}$ & $1.9872 \times 10^{7}$ & $2.7532 \times 10^{7}$ & $1.8993 \times 10^{7}$ & $2.3562 \times 10^{7}$ \\
\hline$\frac{\partial}{\partial x_{j}}\left[q_{j}(\bar{\phi})\right]$ & $1.4881 \times 10^{6}$ & $5.6522 \times 10^{6}$ & $7.0844 \times 10^{6}$ & $7.6108 \times 10^{5}$ & $2.6557 \times 10^{6}$ & $3.3632 \times 10^{6}$ \\
\hline$\frac{\partial}{\partial x_{j}}\left[\sigma_{i j}(\bar{\phi}) \tilde{u}_{i}\right]$ & $1.0697 \times 10^{6}$ & $1.2488 \times 10^{6}$ & $1.3455 \times 10^{6}$ & $5.0522 \times 10^{5}$ & $5.7846 \times 10^{5}$ & $6.2543 \times 10^{5}$ \\
\hline $\bar{S}_{I I I}$ & 0 & $1.5530 \times 10^{7}$ & $2.5564 \times 10^{7}$ & 0 & $1.1828 \times 10^{7}$ & $1.9780 \times 10^{7}$ \\
\hline$\frac{\partial}{\partial x_{j}}\left(\bar{\rho} \zeta_{j}\right)$ & $2.1851 \times 10^{6}$ & $1.1215 \times 10^{7}$ & $1.4102 \times 10^{7}$ & $2.3336 \times 10^{6}$ & $1.2167 \times 10^{7}$ & $1.5300 \times 10^{7}$ \\
\hline$\frac{\partial}{\partial x_{j}}($ Triple $)$ & $6.6728 \times 10^{6}$ & $9.8611 \times 10^{6}$ & $1.0852 \times 10^{7}$ & $7.7174 \times 10^{6}$ & $1.0727 \times 10^{7}$ & $1.1718 \times 10^{7}$ \\
\hline$\frac{\partial}{\partial x_{j}}\left\{\Delta(\bar{p}) \tilde{u}_{j}\right\}$ & $1.5788 \times 10^{6}$ & $2.0174 \times 10^{6}$ & $2.3056 \times 10^{6}$ & $1.6621 \times 10^{6}$ & $1.9663 \times 10^{6}$ & $2.2620 \times 10^{6}$ \\
\hline$\frac{\partial}{\partial x_{j}}\left[\Delta\left(\bar{q}_{j}\right)\right]$ & $6.2555 \times 10^{5}$ & $4.5018 \times 10^{5}$ & $3.9000 \times 10^{5}$ & $4.6745 \times 10^{5}$ & $4.3517 \times 10^{5}$ & $3.6218 \times 10^{5}$ \\
\hline$\frac{\partial}{\partial x_{j}}\left[\Delta\left(\overline{\sigma_{i j} u_{i}}\right)\right]$ & $2.1616 \times 10^{5}$ & $3.1954 \times 10^{5}$ & $3.4383 \times 10^{5}$ & $\begin{array}{l}1.5634 \times 10^{5} \\
\overline{\phi()}-f(\bar{\phi})\end{array}$ & $2.1156 \times 10^{5}$ & $2.3148 \times 10^{5}$ \\
\hline
\end{tabular}

TABLE 8. Magnitude (r.m.s.) of terms in LES equations at transitional times. Units are $\mathrm{J} \mathrm{m}^{-3}$.

are due to the inviscid resolved terms, followed by the SGS stress term. The smallest term (second smallest if $\bar{S}_{I I, i}=0$ ) is that containing the viscous stress differences. Intermediate between the largest and smallest terms are the pressure difference term, the viscous stress term and the FST (if non-zero). For the energy equation (table 8), 


\begin{tabular}{cccccc} 
& \multicolumn{2}{c}{$\bar{\Delta}=4 \Delta x$} & & \multicolumn{2}{c}{$\bar{\Delta}=8 \Delta x$} \\
\cline { 2 - 3 } \cline { 5 - 6 } Species & TP600a2 & TP600a5 & & TP600a2 & TP600a5 \\
$t^{*}$ & 105 & 105 & & 105 & 105 \\
$\frac{\partial}{\partial x_{j}}\left(\bar{\rho} \tilde{Y}_{V} \tilde{u}_{j}\right)$ & $1.9214 \times 10^{2}$ & $2.4156 \times 10^{2}$ & & $1.3079 \times 10^{2}$ & $1.6469 \times 10^{2}$ \\
$\frac{\partial}{\partial x_{j}}\left[j_{V j}(\bar{\phi})\right]$ & $1.0125 \times 10^{1}$ & $1.1959 \times 10^{1}$ & & $4.6568 \times 10^{0}$ & $5.5968 \times 10^{0}$ \\
$\bar{S}_{I}$ & $1.7522 \times 10^{1}$ & $2.9237 \times 10^{1}$ & $1.3301 \times 10^{1}$ & $2.2693 \times 10^{1}$ \\
$\frac{\partial}{\partial x_{j}}\left(\bar{\rho} \eta_{j}\right)$ & $2.1016 \times 10^{1}$ & $2.4744 \times 10^{1}$ & $2.2555 \times 10^{1}$ & $2.6707 \times 10^{1}$ \\
$\frac{\partial}{\partial x_{j}}\left[\Delta\left(\overline{j_{V j}}\right)\right]$ & $5.9755 \times 10^{-1}$ & $7.7168 \times 10^{-1}$ & $6.0994 \times 10^{-1}$ & $5.4061 \times 10^{-1}$
\end{tabular}

TABLE 9. Magnitude (r.m.s.) of terms in LES equations at transitional times. Units are $\mathrm{kg} \mathrm{s}^{-1} \mathrm{~m}^{-3}$.

the largest term is the resolved inviscid term containing the total energy. The smallest terms are the viscous flux term, the heat flux difference term and the viscous flux difference term. The intermediate terms can be divided into two groups, the group of the larger terms comprising the resolved inviscid term containing the pressure, the FST (unless it is null), the triple correlation term and the SGS enthalpy flux term, while the group with the smaller terms consists of the heat flux term and the pressure difference term. For the species equation (table $9 ; Y_{V}$ is null for the SP cases), the largest contributions are due to the inviscid resolved terms. The intermediate terms are the SGS species flux term and the FST; the smallest term is the mass flux difference term. Although there is no definite pattern, the general conclusion is that the ordering of the terms displays dependence on $\bar{\Delta} / \Delta x$ across $M L_{0}$ and $R e_{0}$ values.

In tables 5-9, the assumptions of (4.13)-(4.15) generally lead to the smallest magnitude terms, decisively justifying their neglect. The momentum and species mass source terms are of the same order of magnitude as the resolved viscous stresses and species mass fluxes, respectively, and the energy source term is of the order of the triple correlations, showing that the FSTs are of importance in the LES equations; however, the FSTs are somewhat smaller than the SGS terms in the momentum equation, but of same magnitude as the SGS terms in the energy and species equations. For all the equations, the SGS term tends to be an order of magnitude smaller than the largest, inviscid resolved, term. Thus, from the analysis of the magnitude of various terms, it appears that a successful LES will probably depend mainly on proper resolution of the filtered flow field, then on good models for the SGS fluxes and finally, for TP flows, on the modelling of FSTs. Obviously, to capture the interaction between the drops and the carrier gas, the FSTs are needed, and judging from their magnitude, they should be modelled with care similar to the SGS quantities in the species and energy equations.

\subsection{Simplified LES equations}

Upon incorporating the assumptions of (4.13)-(4.16), which have been validated in $\S 4.2$ and $\S 4.3$, a simpler form of (4.9)-(4.12) is obtained:

$$
\frac{\partial \bar{\rho}}{\partial t}+\frac{\partial\left(\bar{\rho} \tilde{u}_{j}\right)}{\partial x_{j}}=\bar{S}_{I},
$$




$$
\begin{gathered}
\frac{\partial\left(\bar{\rho} \tilde{u}_{i}\right)}{\partial t}+\frac{\partial\left(\bar{\rho} \tilde{u}_{i} \tilde{u}_{j}\right)}{\partial x_{j}}=-\frac{\partial[p(\bar{\phi})]}{\partial x_{i}}+\frac{\partial \sigma_{i j}(\bar{\phi})}{\partial x_{j}}+\bar{S}_{I I, i}-\frac{\partial\left(\bar{\rho} \tau_{i j}\right)}{\partial x_{j}}, \\
\frac{\partial\left(\bar{\rho} \tilde{e}_{t}\right)}{\partial t}+\frac{\partial\left(\bar{\rho} \tilde{e}_{t} \tilde{u}_{j}\right)}{\partial x_{j}}=-\frac{\partial\left[p(\bar{\phi}) \tilde{u}_{j}\right]}{\partial x_{j}}-\frac{\partial q_{j}(\bar{\phi})}{\partial x_{j}}+\frac{\partial\left[\sigma_{i j}(\bar{\phi}) \tilde{u}_{i}\right]}{\partial x_{j}}+\bar{S}_{I I I} \\
-\frac{\partial\left(\bar{\rho} \zeta_{j}\right)}{\partial x_{j}}-\frac{\partial}{\partial x_{j}}\left(\bar{\rho} \tau_{i j} \tilde{u}_{i}\right), \\
\frac{\partial\left(\bar{\rho} \tilde{Y}_{V}\right)}{\partial t}+\frac{\partial\left(\bar{\rho} \tilde{Y}_{V} \tilde{u}_{j}\right)}{\partial x_{j}}=-\frac{\partial j_{V j}(\bar{\phi})}{\partial x_{j}}+\bar{S}_{I}-\frac{\partial\left(\bar{\rho} \eta_{j}\right)}{\partial x_{j}} .
\end{gathered}
$$

(Implicit in (4.17)-(4.20) is the replacement of $\overline{f(\phi)}$ by $f(\bar{\phi})$.) Equations (4.17)-(4.20) for $\bar{\phi}$ contain terms that cannot be computed directly from $\bar{\phi}$ and that therefore must be modelled, namely, (i) the FSTs $\left(\bar{S}_{I}, \bar{S}_{I I, i}, \bar{S}_{I I I}\right)$ and (ii) the SGS fluxes $\left(\tau_{i j}, \zeta_{j}, \eta_{j}\right)$. The a priori modelling of these terms will be undertaken in $\S 6-7$.

\section{Irreversible entropy production for two-phase flows}

A parallel evaluation of the relative importance of source terms entering the physics of the situation is here obtained by deriving the irreversible entropy production expression for TP flows and analysing its budget. The irreversible entropy production is the dissipation, which is of crucial importance in determining the characteristics of turbulent flows because it contains, in particular, the viscous dissipation which measures the loss of mechanical energy to heat and the scalar dissipation which manifests in the mixing.

\subsection{Derivation of entropy equation for two-phase flow with phase change} According to Hirshfelder, Curtis \& Bird (1954), the entropy equation is

$$
\frac{\partial(\rho s)}{\partial t}+\frac{\partial\left(\rho s u_{j}\right)}{\partial x_{j}}=-\frac{\partial \Sigma_{j}}{\partial x_{j}}+g,
$$

where $s$ is the entropy, $\Sigma_{j}$ represents the flux of reversible entropy and $g$ is the rate of irreversible entropy production. In terms of the substantial derivative,

$$
\rho \frac{\mathrm{D} s}{\mathrm{D} t}=-\frac{\partial \Sigma_{j}}{\partial x_{j}}+g-s S_{I} .
$$

From thermodynamics

$$
T \frac{\mathrm{D} s}{\mathrm{D} t}=\frac{\mathrm{D} e}{\mathrm{D} t}+p \frac{\mathrm{D}(1 / \rho)}{\mathrm{D} t}-\mu_{C} \frac{\mathrm{D} Y_{C}}{\mathrm{D} t}-\mu_{V} \frac{\mathrm{D} Y_{V}}{\mathrm{D} t},
$$

where $\mu_{C}$ and $\mu_{V}$ are the chemical potentials of the carrier gas and vapour, respectively; $\mu_{V}=h_{V}-T s_{V}$ where $s_{V}$ is the entropy of the pure vapour. Mathematical manipulations of (5.3) in conjunction with the conservation equations (2.1)-(2.4) and comparing with (5.2) leads to:

$$
\Sigma_{j}=\frac{1}{T}\left(q_{j}-\frac{\rho Y_{C} N_{A}}{m_{C}} V_{C j} \mu_{C}-\frac{\rho Y_{V} N_{A}}{m_{V}} V_{V j} \mu_{V}\right),
$$

where $N_{A}$ is the Avogadro number,

$$
\begin{aligned}
g=\frac{1}{T}\left[S_{I I I}-u_{i} S_{I I, i}\right. & \left.+\left(\frac{1}{2} u_{i} u_{i}-\mu_{V}\right) S_{I}\right] \\
& +\frac{\sigma_{i j}}{T} \frac{\partial u_{i}}{\partial x_{j}}-\frac{q_{j}}{T^{2}} \frac{\partial T}{\partial x_{j}}-j_{C j} \frac{\partial}{\partial x_{j}}\left(\frac{\mu_{C}}{T}\right)-j_{V j} \frac{\partial}{\partial x_{j}}\left(\frac{\mu_{V}}{T}\right) .
\end{aligned}
$$


For an ideal binary mixture of perfect gases (Hirshfelder et al. 1954)

$$
\frac{\partial}{\partial x_{j}}\left(\frac{\mu_{V}}{T}\right)=-\frac{h_{V}}{T^{2}} \frac{\partial T}{\partial x_{j}}+\frac{R_{u}}{m_{V}} \frac{1}{p} \frac{\partial p}{\partial x_{j}}-\frac{R_{u}}{m_{V}} \frac{1}{Y_{V}} \frac{1}{m_{C}\left(\frac{Y_{V}}{m_{V}}+\frac{Y_{C}}{m_{C}}\right)} \frac{\partial Y_{C}}{\partial x_{j}} .
$$

Further manipulations yield the final form of the dissipation as

$$
\begin{gathered}
g=g_{I I I}+g_{I I}+g_{I, k i n e}+g_{I, \text { chpot }}+g_{\text {visc }}+g_{\text {temp }}+g_{\text {mass }}, \\
g_{I I I}=\frac{S_{I I I}}{T}, \quad g_{I I}=-\frac{u_{i} S_{I I, i}}{T}, \quad g_{I, \text { kine }}=\frac{u_{i} u_{i} S_{I} / 2}{T}, \quad g_{I, \text { chpot }}=-\frac{\mu_{V} S_{I}}{T}, \\
g_{\text {visc }}=\frac{\mu}{T}\left(2 S_{i j} S_{i j}-\frac{2}{3} S_{k k} S_{l l}\right)=\frac{2 \mu}{T}\left(S_{i j}-\frac{1}{3} S_{k k} \delta_{i j}\right)\left(S_{i j}-\frac{1}{3} S_{l l} \delta_{i j}\right), \\
g_{\text {temp }}=\frac{\lambda}{T^{2}} \frac{\partial T}{\partial x_{j}} \frac{\partial T}{\partial x_{j}}, \quad g_{\text {mass }}=\frac{R_{C} R_{V}}{Y_{C} Y_{V}\left(R_{V} Y_{V}+R_{C} Y_{C}\right)} \frac{j_{V j} j_{V j}}{\rho D} .
\end{gathered}
$$

The pure vapour entropy, $s_{V}$, is calculated for a perfect gas as

$$
s_{V}=\int C_{p, V}(T) \frac{\mathrm{d} T}{T}-\int R_{V} \frac{\mathrm{d} p}{p},
$$

given a functional form for $C_{p, V}(T)$, where it is required that $s_{V}$ be null at $0 \mathrm{~K}$ (in practice the integration is carried out from $1 \mathrm{~K}$ owing to the logarithmic singularity at $T=0)$. Since here $C_{p, V}$ is constant,

$$
s_{V}=s_{V}^{0}+C_{p, V} \ln \left(T / T^{0}\right)-R_{V} \ln \left(p / p^{0}\right),
$$

where $s_{V}^{0}$ is the reference entropy at the reference temperature $T^{0}$ and pressure $p^{0}$, obtained from integration or tables, and $C_{p, V}=C_{p, V}\left(T^{0}\right)$.

Inspection of (5.7) shows that the gas-phase dissipation has several origins. First, the drops are energy, momentum and mass sources with the resulting dissipation embodied in $g_{I I I}, g_{I I}, g_{I, k i n e}$ and $g_{I, \text { chpot }}$. Note that $g_{I, k i n e}$ and $g_{I, \text { chpot }}$ are entirely due to evaporation, with $g_{I, k i n e}$ due to the gas-phase kinetic energy of the mass evolving from the drops, whereas $g_{I, \text { chpot }}$ is due to its chemical potential. Similar effects are contained in $g_{I I I}$ and $g_{I I}$, but these terms additionally have non-evaporation contributions from the drag on and the heating of the drops. The terms $g_{\text {visc }}, g_{\text {temp }}$ and $g_{\text {mass }}$ contain the flux-related dissipation and are positive semi-definite.

\subsection{Irreversible entropy production at transitional states}

Before undertaking a detailed analysis of the budget of $g$ (5.7), we present contour plots of $g$ for TP600a2 (figure $6 a$ ) and TP600a5 (figure 6b) at the transitional times. As in the flow visualizations of figures 4 and 5, both cases show a qualitatively similar aspect; however, the range of TP600a5 dissipation values is more than twice that of TP600a2. Both layers show significant regions of negative dissipation (darkest blue regions), mainly in the lower stream, but also in small pockets within the mixing region, although the magnitude of the negative dissipation is less than $10 \%$ that of the positive dissipation. Since negative dissipation can arise only from the source-term contributions, this indicates that, at those locations, turbulence increases owing to the presence of the drops. The regions of most intense dissipation are found within the mixing region, and correspond to the high $\rho_{n}$ regions (see figures $4 c$ and $4 d$ ). From the budget of $g$, discussed below, it is apparent that this visual correlation between $g$ and $\rho_{n}$ is due to the $g$ being dominated by the source terms. 


\begin{tabular}{|c|c|c|c|c|c|c|c|c|c|c|}
\hline \multirow[b]{2}{*}{ Term } & \multicolumn{2}{|c|}{ Unfiltered } & \multicolumn{2}{|c|}{$\begin{array}{l}\text { Filtered } \\
\bar{\Delta}=4 \Delta x\end{array}$} & \multicolumn{2}{|c|}{$\begin{array}{c}\text { Difference (SGS) } \\
\bar{\Delta}=4 \Delta x\end{array}$} & \multicolumn{2}{|c|}{$\begin{array}{l}\text { Filtered } \\
\bar{\Delta}=8 \Delta x\end{array}$} & \multicolumn{2}{|c|}{$\begin{array}{c}\text { Difference (SGS) } \\
\bar{\Delta}=8 \Delta x\end{array}$} \\
\hline & Average & r.m.s. & Average & r.m.s. & Average & r.m.s. & Average & r.m.s. & Average & r.m.s. \\
\hline$g_{\text {visc }}$ & 1640 & 3914 & 848 & 1720 & 791 & 2474 & 436 & 814 & 1204 & 3338 \\
\hline$g_{\text {temp }}$ & 13 & 36 & 6 & 13 & 7 & 27 & 3 & 5 & 10 & 33 \\
\hline & 1652 & 3932 & 854 & 1726 & 798 & 2484 & 439 & 817 & & \\
\hline
\end{tabular}

TABLE 10. Entropy production of unfiltered and filtered flow fields, SP600 at $t^{*}=100$. Units are $\mathrm{W} \mathrm{m}^{-3} \mathrm{~K}^{-1}$.

\begin{tabular}{|c|c|c|c|c|c|c|c|c|c|c|}
\hline \multirow[b]{2}{*}{ Term } & \multicolumn{2}{|c|}{ Unfiltered } & \multicolumn{2}{|c|}{$\begin{array}{l}\text { Filtered } \\
\bar{\Delta}=4 \Delta x\end{array}$} & \multicolumn{2}{|c|}{$\begin{array}{c}\text { Difference (SGS) } \\
\bar{\Delta}=4 \Delta x\end{array}$} & \multicolumn{2}{|c|}{$\begin{array}{l}\text { Filtered } \\
\bar{\Delta}=8 \Delta x\end{array}$} & \multicolumn{2}{|c|}{$\begin{array}{c}\text { Difference (SGS) } \\
\bar{\Delta}=8 \Delta x\end{array}$} \\
\hline & Average & r.m.s. & Average & r.m.s. & Average & r.m.s. & Average & r.m.s. & Average & r.m.s. \\
\hline$I I I$ & 13819 & 40010 & 5498 & 49319 & -1680 & 14180 & 17813 & 43664 & -3995 & 19840 \\
\hline$g_{I, \text { chpot }}$ & -8014 & 2493 & -901 & $305^{\circ}$ & & & -10312 & & & 12165 \\
\hline$g_{v i s c}$ & 2658 & & 126 & & 1394 & & 615 & & 2043 & 5599 \\
\hline$g_{I I}$ & -89 & 2405 & -237 & 2752 & 12 & 1030 & -398 & 2559 & 310 & 643 \\
\hline$g_{\text {mass }}$ & 222 & 1302 & 109 & & 11 & 114 & 58 & 172 & 16 & 1243 \\
\hline$g_{\text {temp }}$ & 122 & 40 & 49 & 128 & 73 & 308 & 23 & 52 & 99 & 37 \\
\hline$g_{I, \text { kine }}$ & 57 & 266 & 63 & 306 & -6 & 86 & 72 & 251 & -14 & 13 \\
\hline & 8775 & 19177 & 7735 & 20205 & 1040 & 6810 & 7869 & 17507 & 905 & 9162 \\
\hline
\end{tabular}

TABLE 11. Entropy production of unfiltered and filtered flow fields, TP600a2 at $t^{*}=105$. Units are $\mathrm{W} \mathrm{m}^{-3} \mathrm{~K}^{-1}$.

\begin{tabular}{|c|c|c|c|c|c|c|c|c|c|c|}
\hline \multirow[b]{2}{*}{ Term } & \multicolumn{2}{|c|}{ Unfiltered } & \multicolumn{2}{|c|}{$\begin{array}{l}\text { Filtered } \\
\bar{\Delta}=4 \Delta x\end{array}$} & \multicolumn{2}{|c|}{$\begin{array}{c}\text { Difference (SGS) } \\
\bar{\Delta}=4 \Delta x\end{array}$} & \multicolumn{2}{|c|}{$\begin{array}{l}\text { Filtered } \\
\bar{\Delta}=8 \Delta x\end{array}$} & \multicolumn{2}{|c|}{$\begin{array}{c}\text { Difference (SGS) } \\
\bar{\Delta}=8 \Delta x\end{array}$} \\
\hline & Average & r.m.s. & Average & r.m.s. & Average & r.m.s. & Average & r.m.s. & Average & r.m.s. \\
\hline & 24522 & 66844 & 7020 & 84838 & -4516 & 28197 & 25231 & 80025 & -10710 & 404 \\
\hline chp & -13768 & 424 & -163 & 526 & 25 & 16 & 1964 & & & 23755 \\
\hline isc & 3009 & 69 & 14 & & 160 & & 6 & & & 6142 \\
\hline & 58 & 73 & -466 & 8692 & 54 & 33 & -1070 & 808 & 11 & 5283 \\
\hline nass & 270 & 1189 & 139 & 474 & 131 & 88 & 7 & 233 & 19 & 1074 \\
\hline emp & 136 & 438 & 57 & 156 & 78 & 32 & 28 & 68 & 108 & 399 \\
\hline kine & 114 & 523 & 133 & 603 & -19 & & 158 & 518 & -43 & 293 \\
\hline & 14341 & 29760 & 13986 & 34316 & 355 & 11529 & 15459 & 32093 & -1119 & 16097 \\
\hline
\end{tabular}

TABLE 12. Entropy production of unfiltered and filtered flow fields, TP600a5 at $t^{*}=105$. Units are $\mathrm{W} \mathrm{m}^{-3} \mathrm{~K}^{-1}$.

The strategy of the dissipation analysis is to first compare results obtained for SP600 and TP600a 2 which should reveal the role of the drops, then scrutinize results from TP600a2 and TP600a5 which should elucidate the role of $M L_{0}$ and finally consider this information together with results from TP500a2 and TP500a5 to elucidate the influence of $R e_{0}$. The data are compared from the perspective of the volume average and r.m.s. budget of (5.7). The information is presented in table 10 for SP600, table 11 for TP600a2, table 12 for TP600a5 and table 13 to compare all four TP cases given in table 1 (some information is repeated from tables 11 and 12 for easier visual comparison). The terms in tables 11-13 are arranged according to decreasing r.m.s. 


\begin{tabular}{lrrrrrrrrrr}
\hline & \multicolumn{4}{c}{ Average entropy production } & & \multicolumn{3}{c}{ RMS entropy production } \\
\cline { 2 - 4 } \cline { 7 - 9 } Term & TP500a2 & TP500a5 & TP600a2 & TP600a5 & & TP500a2 & TP500a5 & TP600a2 & TP600a5 \\
$g_{\text {III }}$ & 14730 & 26765 & 13819 & 24522 & & 41990 & 74878 & 40010 & 66844 \\
$g_{I, \text { chpot }}$ & -8512 & -15119 & -8014 & -13768 & & 26037 & 47339 & 24935 & 42435 \\
$g_{\text {visc }}$ & 2966 & 3469 & 2658 & 3009 & & 6878 & 8039 & 6372 & 6979 \\
$g_{I I}$ & -138 & 303 & -89 & 58 & & 2581 & 7392 & 2405 & 7319 \\
$g_{\text {mass }}$ & 255 & 317 & 222 & 270 & & 1194 & 1323 & 1302 & 1189 \\
$g_{\text {temp }}$ & 143 & 153 & 122 & 136 & & 453 & 512 & 401 & 438 \\
$g_{I, \text { kine }}$ & 67 & 128 & 57 & 114 & & 300 & 536 & 266 & 523 \\
$g$ & 9510 & 16017 & 8775 & 14341 & & 20343 & 34440 & 19177 & 29760
\end{tabular}

TABLE 13. Entropy production of transitional flow fields at $t^{*}=105$. Units are $\mathrm{W} \mathrm{m}^{-3} \mathrm{~K}^{-1}$.

of TP500a2 for the DNS (unfiltered) flow field. All tables contain information at the DNS scale and tables 10-12 contain also information at the LES and subgrid scales. The filtered (LES scale) flow field is obtained by filtering the DNS flow field using $\bar{\Delta}=4 \Delta x$ or $\bar{\Delta}=8 \Delta x$ (see $\S 4.2$ ) and the SGS results are calculated by taking the difference between the DNS-field and filtered-field value for each term.

\subsubsection{Comparison of single-phase and two-phase flows, and ordering of DNS terms}

For SP600 (table 10), $g$ is positive, as expected, which means that its effect is to diminish turbulence, and has only two components, $g_{v i s c}$ and $g_{\text {temp }}$. Owing to the compressible nature of the flow, $g_{\text {temp }}$ is not null, however, it is much smaller than $g_{\text {visc }}$ owing to the very small temperature gradients.

For all TP flows (tables 11 and 12), $g$ is positive in average and considerably larger than its SP equivalent, showing that the global effect of the drops on the flow is to increase dissipation and therefore to decrease turbulence. These results extend into the realm of evaporating drops the information obtained from experimental results with solid particles and no phase change by Hetsroni \& Sokolov (1971), Popper, Abuaf \& Hetsroni (1974), Levy \& Lockwood (1981), Modarress, Tan \& Elgobashi (1984), Fleckhaus, Hishida \& Maeda (1987), Tsuji et al. (1988), Gore \& Crowe (1989) and Hardalupas, Taylor \& Whitelaw (1989), which showed that particles smaller than the Kolmogorov length will attenuate turbulence. Owing to the different nature of the present flow fields compared with the solid particle cases, the origin of the leading contributions to $g$ is different. Specifically, for all TP cases, the largest term is $g_{I I I}$, due to the energy contribution of the drops, and is positive on average. The DNS-field average $g_{I I I}$ term increases strongly, but less than proportionally with $M L_{0}$ (1.8 for both $R e_{0}=500$ and $R e_{0}=600$ versus 2.5 increase in $M L_{0}$ ); at both $M L_{0}$, this term decreases slightly with increasing $R e_{0}$. The dominance of $g_{I I I}$ leads to $g$ mimicking its trends, i.e. being more sensitive to $M L_{0}$ than to $R e_{0}$ and decreasing with $R e_{0}$ at both $M L_{0}$. Further, an examination of the DNS-field contributions to $g_{I I I}$ reveals that the $\left(-\dot{m}_{d} h_{V, s}\right)$ contribution is the largest followed by that of $(-Q)$, then by $\left(-F_{i} v_{i}\right)$, and the remaining term with $\left(-\dot{m}_{d} v_{i} v_{i} / 2\right)$ is the smallest.

The second largest term is $g_{I, \text { chpot}}$, which is negative on average. Behaviour similar to that of the average $g_{I I I}$ is observed for the magnitude of the average $g_{I \text {,chpot }}$, as well as for the r.m.s. of both terms. Therefore the entropy production is overwhelmingly of evaporative and thermodynamic, rather than dynamic, nature. Although, for the flow fields considered, $g_{I I I}$ dominates $g_{I, \text { chpot }}$, there may be situations in which $g_{I, \text { chpot }}$ will exceed $g_{I I I}$ in magnitude and, since $g_{I \text {,chpot }}$ is negative, lead to evaporating drops 
enhancing turbulence at the DNS scale. Because $g_{I, c h p o t}$ is null for solid particles, caution must be exercised in generalizing experimental results obtained with solid particles to the different setting of evaporating drops.

The largest term not involving source terms is the viscous dissipation term $g_{\text {visc }}$, which is third in order of importance and is about a factor of seven smaller than the two largest terms. Following $g_{v i s c}$, the next largest term in magnitude is $g_{I I}$. On the DNS field, the magnitude of the average $g_{I I}$ is smaller than the average $g_{v i s c}$ by a factor of 30 (for TP600a2) to 50 (for TP600a5). The influence of $M L_{0}$ is evident in the r.m.s., which at the lower $M L_{0}$ is larger for $g_{\text {visc }}$ than for $g_{I I}$, while at the higher $M L_{0}$ this ordering is reversed. Note that another DNS-field $M L_{0}$ effect is that for TP600a2, $g_{I I}$ is negative on average, whereas for TP600a5, $g_{I I}$ is positive on average. This $g_{I I}$ behaviour is understandable if we consider that $g_{I I} \sim\left[F_{i}+\dot{m}_{d} v_{i}\right]$ in which on average $F_{i}>0$, whereas $\dot{m}_{d} v_{i}<0$. Then it is clear that $g_{I I}>0$ if the drag dominates the masschange term as for TP600a5, whereas $g_{I I}<0$ if the opposite situation exists as for TP600a2. The second largest flux-related term, $g_{\text {mass }}$, increases with $M L_{0}$, indicating that although the mass fraction range is comparable between TP600a2 and TP600a5 (see figure 5), its gradients are stronger for TP600a5. Finally, the two smallest terms for all TP cases are $g_{I, k i n e}$ and $g_{\text {temp }}$, the latter being larger at the lower $M L_{0}$, but smaller at the higher $M L_{0}$ for both $R e_{0}$ in the DNS database.

\subsubsection{Comparison of source-term dissipation at all scales}

Both the filtered-field $g_{I I I}$ and $g_{I, \text { chpot }}$ follow their DNS-field counterparts in being positive and negative, respectively, on average. Notably, the SGS averages have the opposite behaviour, that is, the average SGS $g_{I I I}$ is negative and the average SGS $g_{I, \text { chpot }}$ is positive, as a consequence of the filtered-field dissipation having larger magnitude than that of the DNS. The dependence of $g_{I I I}$ and $g_{I, \text { chpot }}$ on source terms suggests that in LES the correct amount of (global) dissipation cannot be obtained unless the (local) source terms are properly modelled. This conclusion is further borne out by considering the $R e_{0}$ and $M L_{0}$ behaviour of $g$ (see table 13). The DNS-field average and r.m.s. trends in terms of $M L_{0}$ and $R e_{0}$ variation carry over to the LES and subgrid scales for both $g_{I I I}$ and $g_{I, \text { chpot }}$ (except the SGS at $M L_{0}=0.5, \bar{\Delta}=4 \Delta x$ ), but, because of the opposing signs of $g_{I I I}$ and $g_{I, \text { chpot }}$, only to the LES scales for $g$. At the subgrid scales, there is a decline, rather than an increase, in the average $g$ with increasing $M L_{0}$ (independent of $\bar{\Delta} / \Delta x$ ). Notably, for $\bar{\Delta}=8 \Delta x$, the average $g$ is negative. Nonetheless, independent of $\bar{\Delta} / \Delta x$, the SGS r.m.s. increases with $M L_{0}$.

On the filtered field, the average $g_{I I}$ has a sign opposite to that of the average $g_{\text {visc }}$. Although the magnitude of the average $g_{I I}$ is smaller than the average $g_{v i s c}$ (except for TP600a5 at $\bar{\Delta}=8 \Delta x$ ), on the filtered fields the r.m.s. of $g_{I I}$ is larger than that of $g_{v i s c}$. For TP600a2 the $4 \Delta x$-filtered-field average $g_{I I}$ has the same sign as that of the DNS but is about a factor of two larger in magnitude; for TP600a5, the $4 \Delta x$-filtered-field average $g_{I I}$ has the opposite sign and is eight times larger than the DNS. The $8 \Delta x$-filtered-field average $g_{I I}$ has the same sign as the $4 \Delta x$-filtered-field average, but is about twice as large in magnitude independent of $M L_{0}$.

\subsubsection{Comparison of flux-related dissipation at all scales}

For SP600, the dissipation at the LES scale is only about half (at $\bar{\Delta}=4 \Delta x$ ) to a quarter (at $\bar{\Delta}=8 \Delta x$ ) of the DNS. For the SP calculation, the SGS terms increase the dissipation on average; this augmentation is comparable in magnitude to the $4 \Delta x$-filtered-field term but is much larger than the $8 \Delta x$-filtered-field term. The SGS r.m.s. has larger magnitude than the r.m.s. of the filtered-field terms, which is expected 
since the smaller scales are those responsible for the entropy fluctuations associated with turbulence. For both TP600a2 and TP600a5, at all scales, the $g_{v i s c}$ contribution is considerably larger than for SP600, showing dissipation due to the drop effect on the dynamic field in addition to the source-term dissipation. The contribution of the SGS $g_{\text {visc }}$ terms is larger than that at the LES scale both for the average and the r.m.s., thus showing a departure from the SP600 simulation where only the r.m.s. term was larger. Similar to $g_{\text {visc }}$, the contribution of the SGS $g_{\text {mass }}$ and $g_{\text {temp }}$ terms is larger than that at the LES scale for both the average and r.m.s. (except for $g_{\text {mass }}$ at $M L_{0}=0.5$, $\bar{\Delta}=4 \Delta x$ ). The larger $g_{\text {temp }}$ for TP600a2 and TP600a5 compared to SP600 is mainly due to the gradients arising from the temperature difference between the laden and unladen streams (see figure 5).

\subsubsection{Dissipation conclusions}

Overall, the TP flow fields exhibit positive dissipation, and increased dissipation compared to SP flows, meaning that the effect of the drops is to attenuate turbulence. Although the dissipation due to the viscous stresses is significant, the bulk of the dissipation is associated with the drop evaporation, whether for the DNS solution, the filtered flow field or the small scales. The drop-related dissipation depends on the source terms, which in turn depend on the local flow condition encountered by each drop. Furthermore, this dissipation contains contributions, which may be negative or positive, due to each category (mass, momentum, energy) of source term. The global dissipation, an aggregate over the local contributions, was found not to be readily parameterized through initial global characteristics of the flow field, namely $M L_{0}$ and $R e_{0}$, for the few values of these parameters that were studied. Routine detailed simulations of $M L_{0}$ and $R e_{0}$ effects is precluded by the high computational requirements of DNS (see table 1) and motivates the development of a suitable LES methodology with reduced computational demands. Such a methodology would include careful modelling of local source terms, as it seems unlikely that the proper amount of global dissipation could be achieved without capturing the local flow-drop interactions.

\section{Models for filtered source terms}

In LES of TP flow with evaporating drops, the effect of the drops on the gas phase occurs through the filtered source terms (FSTs), $\bar{S}$. Two modelling issues arise in computing $\bar{S}$. First, in calculating $\bar{S}$ through (4.7), the source effect of each drop $\left(S_{d}\right)$ depends on both the drop state $(Z)$ and the unfiltered variables at the drop far field $\left(\psi_{f}\right)$. However, in LES, $\psi_{f}$ is not available and must be modelled from the simulated flow field $(\bar{\phi})$. Secondly, to follow the LES spirit of reducing the resolution requirements, the computational demands for the liquid phase can be diminished by simulating a reduced drop field, here denoted $\bar{Z}$, in which case $\bar{S}$ must now be modelled from $\bar{Z}$. The proposed models for $\bar{S}$ address the two issues just elaborated, namely, the calculation of $\psi_{f}$ from $\bar{\phi}$ and the reduction of the number of tracked drops. Using the available DNS database, such models can be compared a priori against the 'exact' FSTs, i.e. $\bar{S}\left(\psi_{f}, Z\right)$ calculated from the actual unfiltered flow field and the full drop field. When analysing the DNS database, the issue of the reduced drop field may be separated from that of unfiltered variable modelling by calculating FST models using the actual unfiltered variables but with the reduced drop field, or using the modelled unfiltered variables along with the full drop field. By considering an 'ideal' unfiltered variable model that is the same as the actual unfiltered variables, 
and that would thereby accurately incorporate the gas-phase SGS effects, we can designate all FST models according to the underlying unfiltered variable models, which are described next.

\subsection{Models of the unfiltered gas-phase variables at drop locations}

A requirement to model the FSTs is the determination of the gas-phase quantities to be used in computing the source term contribution of the individual drops. We propose here to compute the drop source terms $\left(S_{d}\right)$ using the DNS relations (see $\left.\S 2.2\right)$, which require the knowledge of the drop far field. In the DNS, the flow field $(\phi)$ is computed at the grid points and the drop far field $\left(\psi_{f}\right)$ is computed at drop locations. The DNS procedure to obtain $\psi_{f}$ from $\phi$ can be conceptualized as $\psi(\phi) \stackrel{\mathscr{J}}{\longrightarrow} \psi_{f}$, where $\psi(\phi)$ denotes the functional dependence between primitive $(\psi)$ and conservative $(\phi)$ variables which is used to compute primitive variables at the grid points and $\mathscr{I}$ denotes the interpolation process from grid points to drop locations. The LES requires computing from the simulated flow field $(\bar{\phi})$ a model (subscript $m$ ) for the drop far field, denoted $\psi_{f, m}$, which necessarily entails a reconstruction process $\mathscr{R}^{*}$. Defining the filtered gas-phase primitive variables as $\tilde{\psi}=\left\{\tilde{u}_{i}, \tilde{T}, \tilde{Y}_{V}, \bar{p}\right\}$, then consistent with the gas-phase equations (see $\S 4.1-4.2), \tilde{\psi}(\bar{\phi})$ takes the same form as $\psi(\phi)$. Two possible LES procedures for calculating $\psi_{f, m}$ are: (i) $\tilde{\psi}(\bar{\phi}) \stackrel{\mathscr{R}^{*}}{\longrightarrow} \psi_{m} \stackrel{\mathscr{I}}{\longrightarrow} \psi_{f, m}$ and (ii) $\tilde{\psi}(\bar{\phi}) \stackrel{\mathscr{I}}{\longrightarrow}$ $\tilde{\psi}_{f} \stackrel{\mathscr{R}^{*}}{\longrightarrow} \psi_{f, m}$. Higher accuracy is attained by computing gas-phase quantities at the (fixed) grid points rather than at the (moving) drop locations, hence we adopt procedure (i), in which the unfiltered variable models computed at the grid points are denoted as $\psi_{m}$.

\subsubsection{Description of unfiltered variable models}

The $\psi_{m}$ that we consider are those for which Okong'o \& Bellan (2000) conducted an a priori study, which, however, did not examine the FSTs resulting from these $\psi_{m}$. All the models of Okong'o \& Bellan (2000) are based on the filtered flow field $\tilde{\psi}$, and some models also use the SGS standard deviation, $\sigma_{S G S}$, where $\sigma_{S G S}^{2}(\tilde{\psi})=\widetilde{\psi^{2}}-\tilde{\psi}^{2}$ (see (7.1) and (7.2)). We will compare four $\psi_{m}$, denoted as:

(i) Ideal, which precisely replicates $\psi$, i.e. $\psi_{m}=\psi$. Although such a model cannot be constructed in LES, in the a priori analysis it allows us to assess the best-case scenario in which errors due to modelling the unfiltered flow field are eliminated.

(ii) Baseline, which uses the filtered field, i.e. $\psi_{m}=\tilde{\psi}$. This model neglects SGS effects on drop evolution.

(iii) Random, which uses a random function for $\psi_{m}$, specifically, a Gaussian with mean $\tilde{\psi}$ and standard deviation $\sigma_{S G S}$. This model is based on the a priori analysis of Okong'o \& Bellan (2000), which showed this distribution to be applicable to the gas-phase variables within the filtering volumes.

(iv) Deterministic, which uses the analytically derived model of Okong'o \& Bellan (2000) wherein

$$
\psi_{m}=\tilde{\psi}-\bar{\sigma} \operatorname{sign}\left(\nabla^{2} \tilde{\psi}\right)
$$

with the filtered standard deviation $\sigma=\sqrt{\overline{\sigma^{2}}}$ modelled as

$$
\overline{\sigma^{2}}=(\sqrt{\overline{\psi \psi}}-\sqrt{\overline{\tilde{\psi} \tilde{\psi}}})^{2}=\left(\sqrt{\sigma_{S G S}^{2}+\tilde{\psi} \tilde{\psi}}-\sqrt{\overline{\tilde{\psi} \tilde{\psi}}}\right)^{2} .
$$

In LES, both $\sigma_{S G S}$ and some components of $\tilde{\psi}$ (specifically, $\tilde{T}$ and $\bar{p}$; see $\S 4.2$ ) must be modelled; however, we here use the actual $\tilde{\psi}$ and $\sigma_{S G S}$ as calculated from 


\begin{tabular}{lccccccc} 
& \multicolumn{3}{c}{$\bar{\Delta}=4 \Delta x$} & & \multicolumn{3}{c}{$\bar{\Delta}=8 \Delta x$} \\
\cline { 2 - 4 } Model & Baseline & Random & Deterministic & & Baseline & Random & Deterministic \\
$u_{1, f}$ & 1.002 & 0.999 & 1.002 & & 1.005 & 0.998 & 1.001 \\
$u_{2, f}$ & 1.020 & 0.967 & 0.998 & & 1.041 & 0.913 & 0.992 \\
$u_{3, f}$ & 1.076 & 0.890 & 0.997 & & 1.175 & 0.712 & 0.970 \\
$T_{f}$ & 0.999 & 0.999 & 1.000 & & 0.999 & 0.998 & 0.999 \\
$Y_{V, f}$ & 1.006 & 1.003 & 1.002 & & 1.014 & 1.008 & 1.007 \\
$p_{f}$ & 1.000 & 1.000 & 1.000 & & 1.001 & 1.001 & 1.000
\end{tabular}

TABLE 14. Slopes from least-squares fit of exact to modelled quantities, slope $=$ exact $/ \mathrm{model}$, TP600a2 at $t^{*}=105$ : unfiltered primitive variables interpolated to drop locations.

\begin{tabular}{lccccccc} 
& \multicolumn{3}{c}{$\bar{\Delta}=4 \Delta x$} & & \multicolumn{3}{c}{$\bar{\Delta}=8 \Delta x$} \\
\cline { 2 - 4 } \cline { 6 - 7 } Model & Baseline & Random & Deterministic & & Baseline & Random & Deterministic \\
$S_{I, d}$ & 0.949 & 0.847 & 0.991 & & 0.863 & 0.729 & 0.926 \\
$S_{I I, 1, d}$ & 0.839 & 0.440 & 0.939 & & 0.556 & 0.229 & 0.686 \\
$S_{I I, 2, d}$ & 0.843 & 0.355 & 0.929 & & 0.498 & 0.156 & 0.624 \\
$S_{I I, 3, d}$ & 0.787 & 0.288 & 0.889 & & 0.390 & 0.113 & 0.529 \\
$S_{I I I, d}$ & 0.948 & 0.798 & 0.993 & & 0.864 & 0.669 & 0.936
\end{tabular}

TABLE 15. Slopes from least-squares fit of exact to modelled quantities, slope $=$ exact $/$ model, TP600a2 at $t^{*}=105$ : drop source terms.

\begin{tabular}{cccccccc} 
& \multicolumn{3}{c}{$\bar{\Delta}=4 \Delta x$} & & \multicolumn{3}{c}{$\bar{\Delta}=8 \Delta x$} \\
\cline { 2 - 4 } \cline { 6 - 7 } Model & Baseline & Random & Deterministic & & Baseline & Random & Deterministic \\
$\bar{S}_{I}$ & 0.879 & 0.855 & 0.974 & & 0.752 & 0.726 & 0.861 \\
$\bar{S}_{I I, 1}$ & 0.824 & 0.787 & 0.949 & & 0.610 & 0.583 & 0.783 \\
$\bar{S}_{I I, 2}$ & 0.866 & 0.814 & 0.965 & & 0.619 & 0.585 & 0.810 \\
$\bar{S}_{I I, 3}$ & 0.813 & 0.745 & 0.901 & & 0.447 & 0.445 & 0.660 \\
$\bar{S}_{I I I}$ & 0.872 & 0.844 & 0.981 & & 0.738 & 0.711 & 0.871
\end{tabular}

TABLE 16. Slopes from least-squares fit of exact to modelled quantities, slope $=$ exact $/$ model, TP600a2 at $t^{*}=105$ : filtered source terms.

the DNS database in order to decouple the assessment of the reconstruction process from the issue of modelling $\tilde{\psi}$ and $\sigma_{S G S}$. The modelling of $\sigma_{S G S}$ is discussed in $\S 7$.

The analysis here consists of using $\psi_{m}$ to compute the drop far-field primitive variables $\left(\psi_{f, m}\right)$, the source term contribution of individual drops $\left(S_{d, m}=S_{d}\left(\psi_{f, m}, Z\right)\right)$ and the FSTs $\left(\bar{S}_{m}=\bar{S}\left(\psi_{f, m}, Z\right)\right)$. For brevity, $\psi_{f, m}, S_{d, m}$ and $\bar{S}_{m}$ will be designated according to $\psi_{m}$ as ideal, baseline, random or deterministic models. The results from the models will be compared to the 'exact' (i.e. DNS flow field) quantities $\left(\psi_{f}, S_{d}\right.$ and $\bar{S}$ ). Note that, because we are still considering the physical drop field, the ideal model here leads to the exact $\psi_{f}, S_{d}$ and $\bar{S}$; therefore, only the other three models will be assessed. We use the same method as in the DNS to interpolate the gas phase variables to the drop locations and to calculate $S_{d}$. Both $\bar{S}_{m}$ and $\bar{S}$ are calculated through (4.7), that is, at this juncture the exclusive difference between them is in the use of $\psi_{m}$ instead of $\psi$. 


\subsubsection{Analysis of unfiltered variable models}

Comparisons of two quantities defined at all grid points, $\mathscr{X}$ (the model) and $\mathscr{Y}$ (the exact quantity), are performed using the LS fit of $\mathscr{Y}=b \mathscr{X}$ which leads to the slope and the correlation of (3.6) and (3.7). (For drop-based quantities, the averaging is performed over the ensemble of all relevant drops.) The analysis is here performed for TP600a 2 at the transitional state and is based on the filtered flow fields obtained at two different filter widths, $\bar{\Delta}=4 \Delta x$ and $\bar{\Delta}=8 \Delta x$ (see $\S 4.2$ ). Slopes $b$, according to (3.6), are tabulated in table 14 (for $\psi_{f}$ ), table 15 (for $S_{d}$ ) and table 16 (for $\bar{S}$ ). The a priori analysis is restricted to a single simulation and uses TP600a2 because it has stronger individual-drop evaporation (see $\S 3.1$ and figure 3 ) and is thus expected to lead to more robust models.

Displayed in table 14 is the assessment of the baseline, random and deterministic $\psi_{m}$ in their prediction of $\psi_{f}$. For all models, the largest discrepancy is in the modelling of $u_{3, f}$, with errors (measured according to the deviation from the optimum slope of unity) of $8 \%, 11 \%$ and $0.3 \%$ for the baseline, random and deterministic $\psi_{m}$, respectively, when $\bar{\Delta}=4 \Delta x$. When $\bar{\Delta}=8 \Delta x$, the corresponding errors are $17 \%, 29 \%$ and $3 \%$. Therefore, the deterministic $\psi_{f, m}$ is much better than the baseline $\psi_{f, m}$, while the random $\psi_{f, m}$ is slightly worse than the baseline. Next, $\psi_{f}$ and the three $\psi_{f, m}$ are used in (2.27) to calculate drop source terms $\left(S_{d}\right.$ or $\left.S_{d, m}\right)$; the slopes comparing $S_{d}$ to the model predictions are given in table 15. The largest discrepancy is in the modelling of $S_{I I, 3, d}$, with errors of $21 \%, 71 \%$ and $11 \%$ for the baseline, random and deterministic models, respectively, for $\bar{\Delta}=4 \Delta x$, and as much as $61 \%, 89 \%$ and $47 \%$ for $\bar{\Delta}=8 \Delta x$. The behaviour mimics that of table 14 , where $u_{3, f}$ was the least accurately modelled component of $\psi_{f}$. However, the modelling errors are much larger in $S_{d, m}$ than in $\psi_{f, m}$; the random model performs quite poorly while the deterministic model performs best. All the slopes are less than unity, meaning that the source terms will on average be overpredicted.

Finally, the FSTs $(\bar{S})$, which are the quantities entering the LES equations, are shown in table 16; these are computed by summing the (exact or modelled) drop source terms of table 15 over all drops within each filtering volume. Similar to table 15 , the largest discrepancy is in the modelling of $\bar{S}_{I I, 3}$, with errors of $19 \%, 26 \%$ and $10 \%$ for the baseline, random and deterministic models, respectively, when $\bar{\Delta}=4 \Delta x$, and as much as $52 \%, 55 \%$ and $34 \%$ for $\bar{\Delta}=8 \Delta x$. As shown in table $7, \bar{S}_{I I, 3}$ can exceed the resolved viscous stress term in the LES $x_{3}$-momentum equation, and thus errors in modelling $\bar{S}_{I I, 3}$ may substantially affect the success of LES. The smallest discrepancy is in the modelling of $\bar{S}_{I}$ and is close to that in modelling $\bar{S}_{I I I}$. In comparing the results for $S_{d, m}$ and $\bar{S}_{m}$, we note that for $\bar{S}$ all the slopes for the random model tend to increase with respect to those of $S_{d}$ (except for $\bar{S}_{I}$ ), whereas for the other two models, only the slopes of some momentum source terms $\left(S_{I I, 2}\right.$ and $\left.S_{I I, 3}\right)$ consistently tend to increase. The deterministic model $\bar{S}_{m}$ is still the best, followed by the baseline model, but the random model is now comparable to the baseline model. Similar to what was seen for $S_{d}$, for $\bar{S}$ all the slopes are less than unity, meaning that $\bar{S}$ will on average be overpredicted. Notably, the slopes for $\bar{S}$ given in table 16 are considerably lower than those for $\psi_{f}$ given in table 14, and all the models show definite deterioration with increased $\bar{\Delta}$.

This analysis indicates that with exact $\sigma_{S G S}$, the deterministic model is best while the baseline model is generally no worse, and usually better than the more sophisticated random model. This can partly be attributed to the nonlinear nature of the relationship between $\psi_{f}$ and $S_{d}$, since small discrepancies in $\psi_{f, m}$ seem to lead to much larger 
discrepancies in $S_{d, m}$. The errors in $\bar{S}_{m}$ are considerably larger than those in $\psi_{f, m}$. Furthermore, the magnitude of the error in $\psi_{f, m}$ shows no predictive ability for the error in the resulting $\bar{S}_{m}$. Apparently, the filter width is the main determinant regarding the accuracy of $\bar{S}_{m}$, since the errors consistently and dramatically increase with $\bar{\Delta}$. For the deterministic model, it is clear that the $\sigma_{S G S}$ model will impact on the $\bar{S}_{m}$ predictions. For the random model, it is also clear that concentrating on an accurate $\sigma_{S G S}$ model is an incorrect strategy since with the exact $\sigma_{S G S}$ the random model predictions are very close to those of the baseline model which is devoid of $\sigma_{S G S}$.

\subsection{Computational-drop modelling}

Having studied the effect of the unfiltered-variable modelling on the source terms, we now turn to the issue of reducing the number of computed drops. In LES, the only information that would be available from experiments is the total number of drops; small-scale DNS-type drop distribution is not currently measurable. Thus, in LES we must make a 'blind' choice in representing the physical drops $(Z)$ by a computational drop field $(\bar{Z})$. In this blind formulation, each computational drop represents a number, $N_{R}$, of physical drops. For simplicity, we will assume that $N_{R}$ is the same for all drops, that is, $N_{R}=N_{\alpha} / N_{\beta}$ is the ratio between the number of physical drops, $N_{\alpha}$, and the number of computational drops, $N_{\beta}$. The FSTs are then computed for the $N_{\beta}$ drops, and scaled by $N_{R}$ leading to

$$
\bar{S}_{m}\left(\psi_{f, m}(\bar{\phi}), \bar{Z}\right)=N_{R} \sum_{\beta} \frac{1}{V_{f}}\left[S_{d}\left(\psi_{f, m}(\bar{\phi}), \bar{Z}\left(N_{R}\right)\right)\right]_{\beta} .
$$

As in (4.7), the summation is over the drops within the filtering volume $V_{f}$, but now over a representative drop field $(\bar{Z})$ rather than the physical drops $(Z)$. The source term contributions for each computational drop are computed in the same manner as for physical drops, that is, the representative nature of each drop is entirely embodied in the parameter $N_{R}$. The unfiltered variables $\left(\psi_{f}\right)$ required for calculating each drop's source term contributions are modelled $\left(\psi_{f, m}\right)$, as described in $\S 6.1$. The modelled FSTs $\left(\bar{S}_{m}\right)$ are next compared to the exact (i.e. DNS) FSTs $\bar{S}\left(\psi_{f}, Z\right)$. Note that the ideal $\psi_{f, m}$ leads to the exact $\bar{S}$ only on the physical drop field $\left(N_{R}=1\right)$. The computational drops are here selected from the DNS drops by extracting them from the array containing $Z$ with a stride of $N_{R}$; each computational drop retains the physical drop characteristics (mass, location, velocity, temperature). Thus, the mass loading may not be strictly conserved between DNS and LES.

\subsubsection{Computational-drop-model analysis at transitional states}

To illustrate the effect of increasing $N_{R}$ (decreasing the number of computational drops), in figure 7 are the $\left(x_{1}, x_{3}\right)$ homogeneous-plane averages and r.m.s. of $\bar{S}_{I}$ and its models. The results for $\bar{S}_{m}$ are labelled according to the $\psi_{m}$ used (ideal, baseline, random, deterministic), while those for $\bar{S}$ are labelled as exact. These plots for $N_{R}=1,8$ and 64 are not intended to be typical, but rather to visualize the global comparisons to be presented below. Clearly, in the middle of the layer there is strong evaporation, as indicated by the average, $\bar{S}_{I}$ being positive. In the lower stream, on average, $\bar{S}_{I}$ is negative, indicating net condensation; further scrutiny revealed that at some locations there is also evaporation. As shown by the small r.m.s. in the lower stream, the magnitude of condensation/evaporation is small. Returning to the issue of computational-drop modelling, figure 7 shows that $\bar{S}_{I}$ is generally overpredicted by 

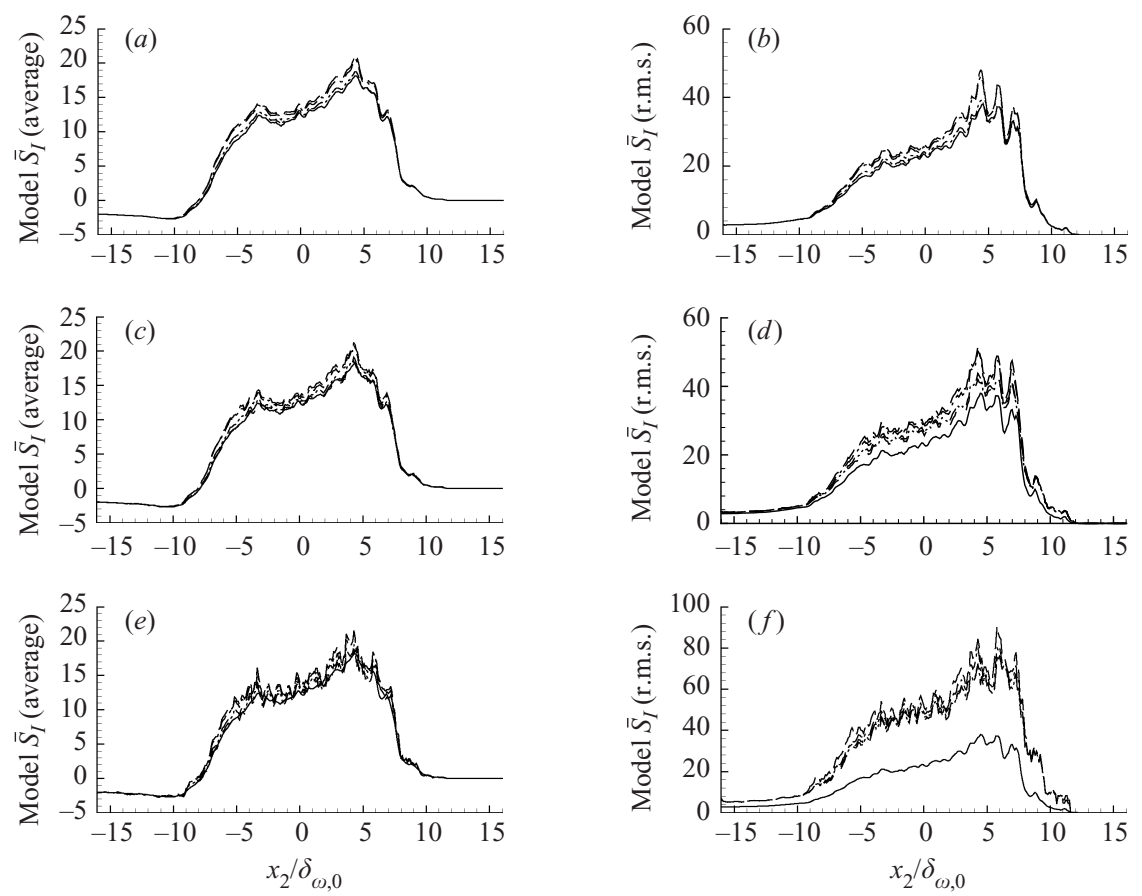

FIGURE 7. Homogeneous $\left(x_{1}, x_{3}\right)$-plane $(a, c, e)$ averages and $(b, d, f)$ r.m.s. of $\bar{S}_{I}$ models, TP600a2 at $t^{*}=105, \bar{\Delta}=4 \Delta x:(a, b) N_{R}=1,(c, d) N_{R}=8,(e, f) N_{R}=64$. The filtered source term models are designated - -, ideal; ---, baseline; --, random or -..-, deterministic according to $\psi_{m}$, the model used for the unfiltered gas-phase variables. - , exact. $N_{R}$ is the ratio of the number of physical drops to the number of computational drops.

the models. Whereas on average, the models do not seem to show much dependence on $N_{R}$, the r.m.s. shows a clear deterioration with $N_{R}$. The greatest error in $\bar{S}_{I, m}$ occurs in the middle of the layer where the strongest evaporation occurs and where the filtered flow field differs most from the unfiltered flow field. In contrast, near the boundaries the filtered flow field is almost identical to the unfiltered one. Since the models are relatively more accurate at the lower stream boundary, where the non-zero $\bar{S}_{I}$ shows that the drops are still evaporating/condensing, the indications are that drop evaporation is not by itself the cause of errors in the FST models. Rather, errors arise from the imprecision in representing the physical drop field by the computational drop field, since the ideal model (which uses the actual unfiltered variables) gives the same results as the other models near the lower boundary. Given that the errors due to modelling the unfiltered flow field (see §6.1) are unavoidable in LES, we want to determine the conditions under which the additional errors introduced by the computational-drop modelling are acceptably small.

To quantify the effect of increasing $N_{R}$, slopes equivalent to those in table 16 are plotted in figures 8 and 9 for $\bar{S}_{I}$ (figures $8 a$ and $8 b$ ), $\bar{S}_{I I I}$ (figures $8 c$ and $8 d$ ), $\bar{S}_{I I, 1}$ (figures $9 a$ and $9 b$ ), $\bar{S}_{I I, 2}$ (figures $9 c$ and $9 d$ ), and $\bar{S}_{I I, 3}$ (figures $9 e$ and $9 f$ ), for $\bar{\Delta}$ of $4 \Delta x$ and $8 \Delta x$. These figures are for $N_{R}=1,2,4,8,16,32,64$ for all FST models; all quantities are compared to $\bar{S}$ computed at the corresponding $\bar{\Delta}$. (Note: the slopes for $N_{R}=1$ were previously presented in table 16.) In figures 8 and 9 , all the slopes are smaller than unity, meaning that all the models overestimate the source terms. Generally, the deterministic model outperforms the baseline model, with errors almost 

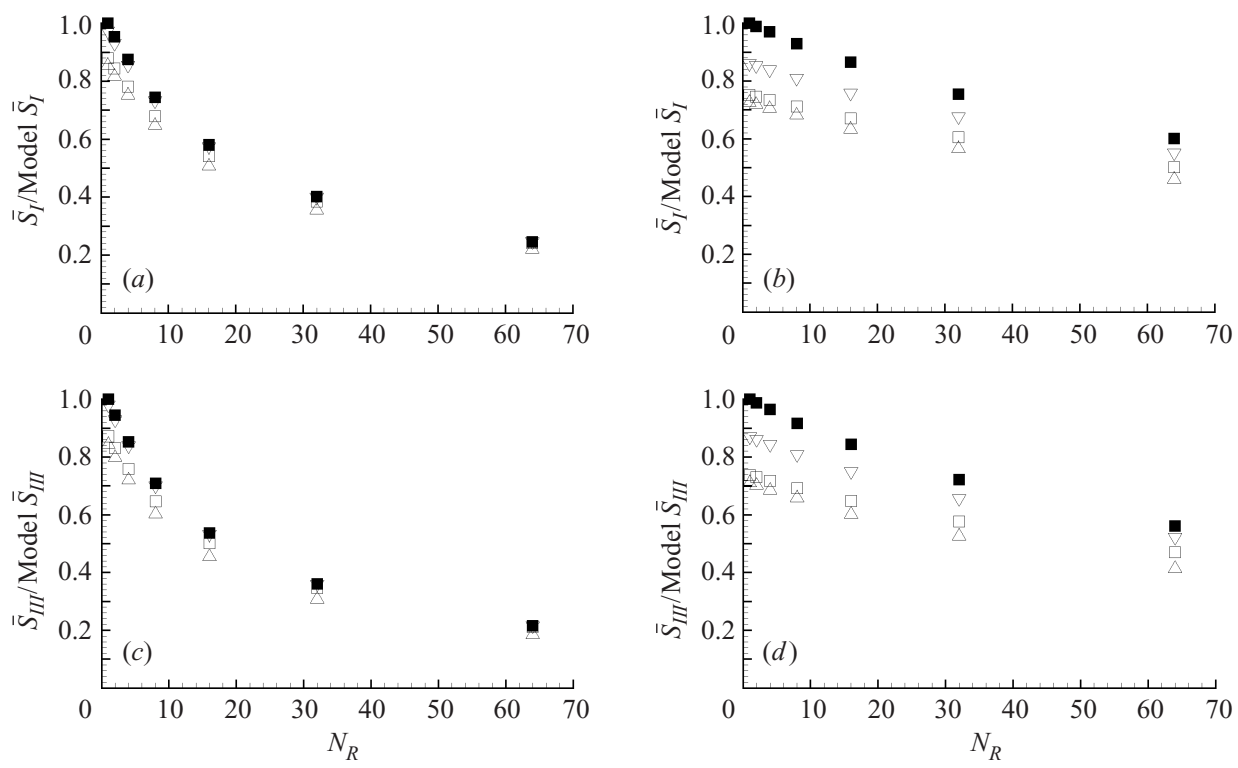

FiguRE 8. Slopes from least-squares fit of exact to modelled filtered source terms, TP600a2 at $t^{*}=105:(a, b) \bar{S}_{I},(c, d) \bar{S}_{I I I}$, for $(a, c) \bar{\Delta}=4 \Delta x$ and $(b, d) \bar{\Delta}=8 \Delta x$. See caption of figure 7 for additional information. $\mathbf{\square}$, ideal $\psi_{m} ; \square$, baseline $\psi_{m} ; \Delta$, random $\psi_{m} ; \nabla$, deterministic $\psi_{m}$.
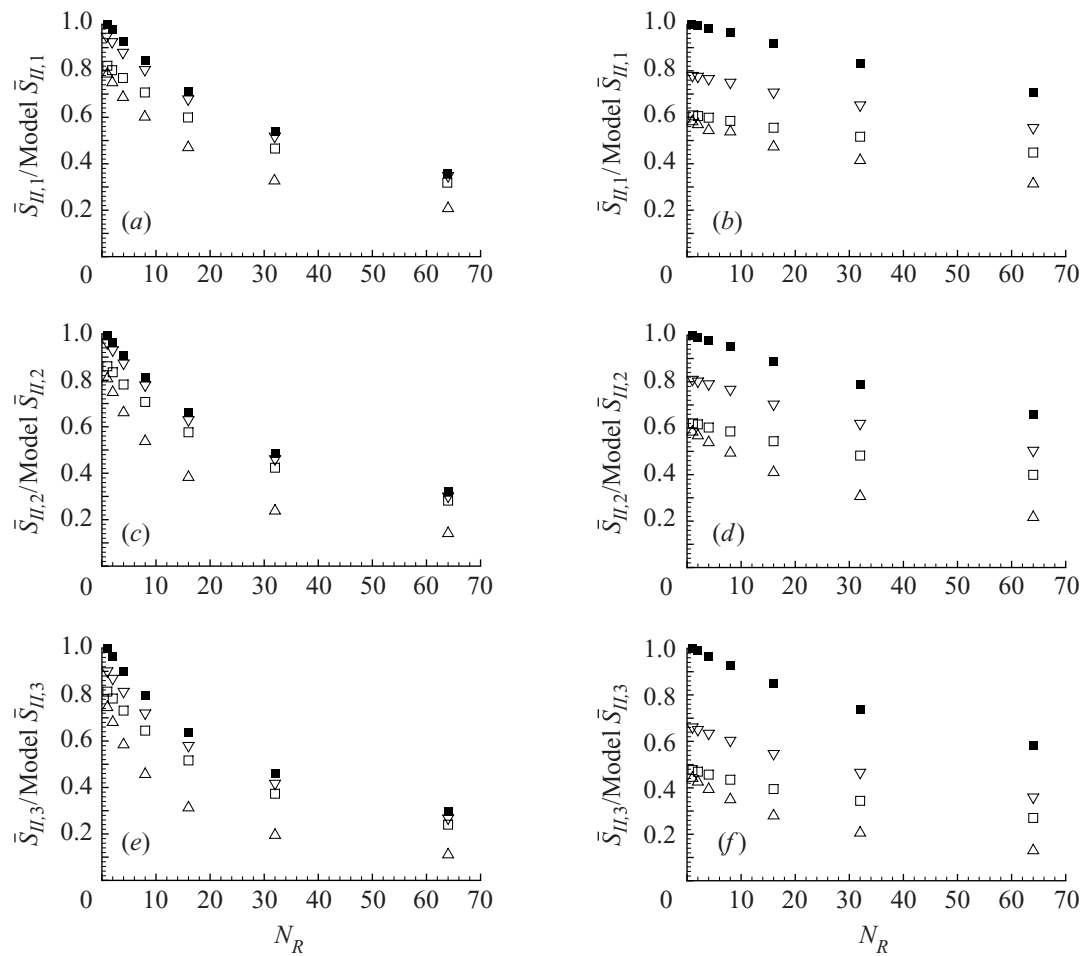

FIGURE 9. Slopes from least-squares fit of exact to modelled filtered source terms, TP600a2 at $t^{*}=105:(a, b) \bar{S}_{I I, 1},(c, d) \bar{S}_{I I, 2},(e, f) \bar{S}_{I I, 3}$ for $(a, c, e) \bar{\Delta}=4 \Delta x$ and $(b, d, f) \bar{\Delta}=8 \Delta x$. See caption of figure 7 for additional information. $\boldsymbol{\square}$, ideal $\psi_{m} ; \square$, baseline $\psi_{m} ; \Delta$, random $\psi_{m} ; \nabla$, deterministic $\psi_{m}$. 
halfway between those of the baseline and ideal models, and the random model is the worst. Consistently, the ideal model gives the best prediction for $\bar{S}$; its relative superiority is significant at the larger filter width, and at the smaller filter width when $N_{R}$ is small. For the smaller filter width at larger $N_{R}$, all the models give similar predictions. The accuracy of the models declines with $N_{R}$, but not at the same rate for all source terms as $\bar{S}_{I I I}$ seems to be best predicted whereas $\bar{S}_{I I, 3}$ seems to be the worst predicted with the strongest error growth with increasing $N_{R}$.

From figures 8 and 9 , it can be concluded that for small $N_{R}$ (more computational drops), the inaccuracies in $\psi_{f, m}$ are a much stronger source of error than is the effect of computational-drop modelling $\left(N_{R}>1\right)$. When using $\psi_{f}$, the ideal model $\bar{S}_{m}$ improves for fixed $N_{R}$ as $\bar{\Delta}$ is increased from $4 \Delta x$ to $8 \Delta x$ since the filtering volume is increased and the number of drops within each filtering volume is accordingly increased, giving a better accuracy. The opposite trend is observed with all the other $\bar{S}_{m}$ because as $\bar{\Delta}$ increases, information is lost during the gas-phase variable modelling, resulting in decreasing accuracy (as $\bar{\Delta}$ increases, $\tilde{\psi}$ is more unlike $\psi$, see table 14). However, for large $N_{R}$ (fewer computational drops), larger than about 8 , the effect of having few drops dominates, as can be seen by the increasing convergence of the slopes from the ideal $\bar{S}_{m}$ to those from the other $\bar{S}_{m}$ for larger $N_{R}$. Figures 8 and 9 also show the effect of the nonlinear relationship between $\psi_{f, m}$ and $\bar{S}_{m}$ in that a proportional reduction in grid resolution and number of drops does not give the same error in the FSTs. That is, an eight-fold increase in the filtering volume, by increasing $\bar{\Delta}$ from $4 \Delta x$ to $8 \Delta x$, and a concomitant eight-fold decrease in the number of drops $\left(N_{R}=1,2,4,8\right.$ compared to $N_{R}=8,16,32,64$, respectively), that is, preserving the number of drops in each filtering volume, does not maintain the error in $\bar{S}_{m}$. In quantifying the $\bar{S}_{m}$ error through the maximal percentage error (greatest deviation from unity over the five source terms, multiplied by 100), the maximal $\bar{S}_{m}$ error was found to be in $\bar{S}_{I I, 3}$ for the baseline, random and deterministic models. Maximal percentage errors obtained from the data in figures $9(e)$ and $9(f)$ show that, for the baseline model, the error for $N_{R}=8$ and $\bar{\Delta}=8 \Delta x$ is three times that for $N_{R}=1$ and $\bar{\Delta}=4 \Delta x$, whereas the error for $N_{R}=64$ and $\bar{\Delta}=8 \Delta x$ is twice that for $N_{R}=8$ and $\bar{\Delta}=4 \Delta x$. A similar trend of decreasing error ratio with increasing $N_{R}$ is observed for the other models. When proportionally increasing $N_{R}$ and the filter volume, the largest loss of accuracy is experienced with the deterministic model, which is the most accurate, while the smallest relative error is with the random model, which is the least accurate.

In LES, the effect of modelling $\psi_{f}$ is unavoidable and most probably $\bar{\Delta}$ would have been selected according to the gas-phase resolution requirements; once $\bar{\Delta}$ is selected, the accuracy of the calculation will decrease with increasing $N_{R}$, independent of the model. This means that if large errors are computationally acceptable (i.e. order of magnitude calculations), a large $N_{R}$ and large $\bar{\Delta}$ are acceptable because the error will be comparable to that for large $N_{R}$ and small $\bar{\Delta}$; that is, as $N_{R}$ increases, the error becomes independent of $\bar{\Delta}$.

\subsubsection{Computational-drop-model analysis at pre-transitional states}

The above results concerning the computational drops were obtained at a transitional state. It is pertinent to inquire whether the overprediction of the FSTs is unique to this time station, or rather a general occurrence. To this end, the analysis of $\bar{S}_{m}$ was repeated at $t^{*}=20,45$ and 80 , corresponding to time stations before the first pairing, between the first and second pairings and at the end of the second pairing. The results are plotted along with those at $t^{*}=105$, in terms of the maximal percentage error (greatest deviation from unity over the five source terms, multiplied 

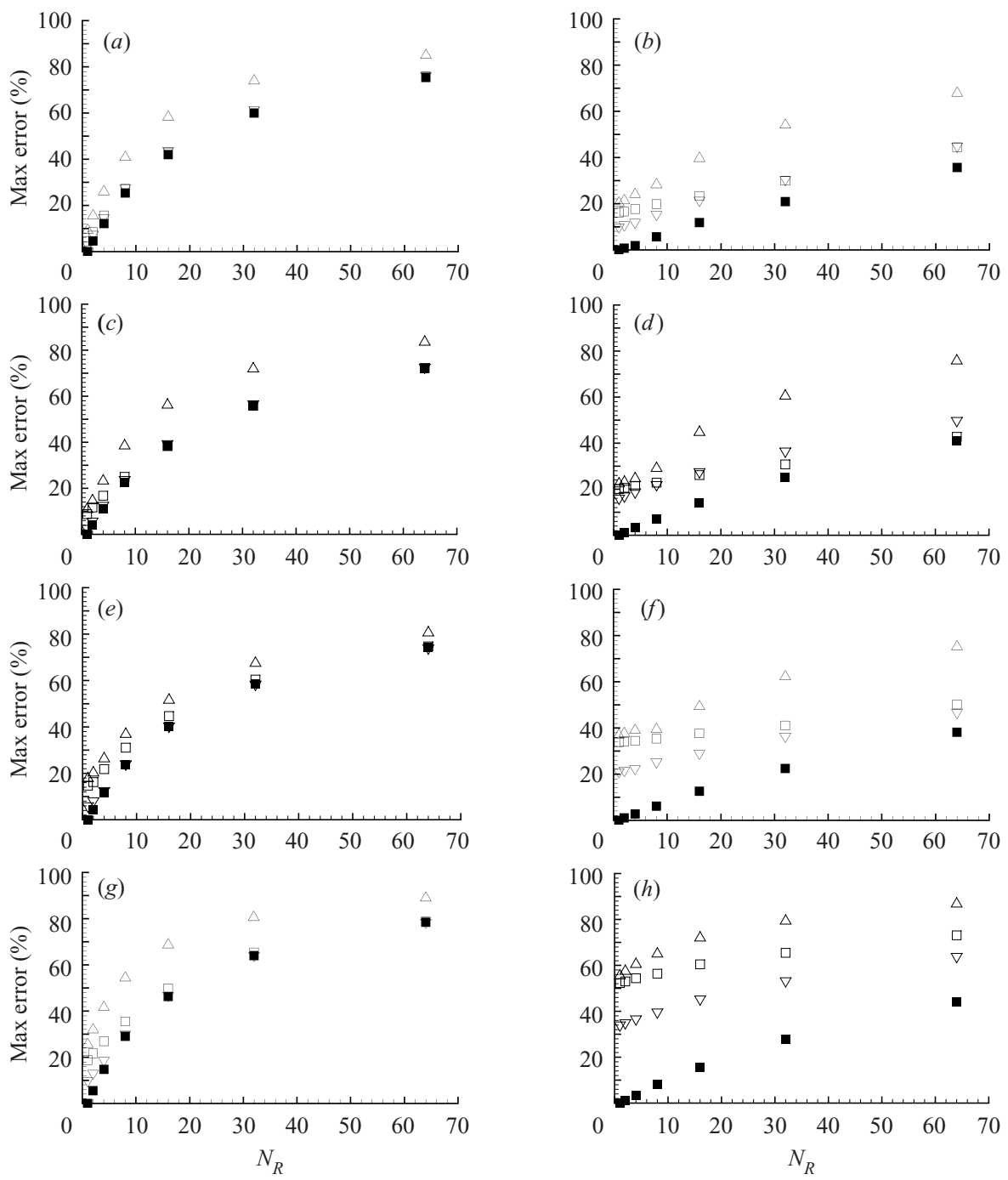

FIGURE 10. Maximal percentage error in filtered source term models, TP600a2 at: $(a, b)$ $t^{*}=20,(c, d) t^{*}=45,(e, f) t^{*}=80,(g, h) t^{*}=105$ for $(a, c, e, g) \bar{\Delta}=4 \Delta x$ and $(b, d, f, h) \bar{\Delta}=$ $8 \Delta x$. See caption of figure 8 for additional information.

by 100) in figure 10 for $t^{*}=20$ (figures $10 a$ and $10 b$ ), $t^{*}=45$ (figures $10 c$ and $10 d$ ), $t^{*}=80$ (figures $10 e$ and $10 f$ ) and $t^{*}=105$ (figures $10 \mathrm{~g}$ and $10 \mathrm{~h}$ ) at $\bar{\Delta}$ of $4 \Delta x$ and $8 \Delta x$. Figures $10(g)$ and $10(h)$ represent the data in figures 8 and 9 (at $\left.t^{*}=105\right)$, and the remarks made when discussing those figures are even more evident: (i) the effect of modelling $\psi_{f}$ is dominant at the larger $\vec{\Delta}$, where the error is initially large but not so sensitive to $N_{R}$, (ii) the effect of modelling $\psi_{f}$ is significant at the smaller $\bar{\Delta}$ for smaller $N_{R}$ but not at larger $N_{R}$, (iii) the deterministic model performs best, followed closely by the baseline model, with the random model giving the worst predictions, and (iv) decreasing the number of computational drops proportionally to the increase in filtering volume size does not necessarily maintain the filtering error. In considering the pre-transitional time stations, the trends at a given $N_{R}$ seem to be as follows: (i) for the smaller $\bar{\Delta}$, the error seems insensitive to time up to $t^{*}=80$ and then exhibits a 
small growth, except for the lowest $N_{R}=1$ or 2 where the error clearly grows with time, and (ii) for the larger $\bar{\Delta}$, the error generally grows with time, with more pronounced error growth at smaller values of $N_{R}$.

\subsubsection{Computational-drop modelling conclusions}

From this a priori study, the indications are that the unfiltered flow field models perform better for smaller filter widths; however, only at small values of $N_{R}$ (below about 8 for $\bar{\Delta}=4 \Delta x$ ) does this translate into improved accuracy of FST models. On the other hand, for larger filter widths, there is little to be gained by using small $N_{R}$, since the error growth with $N_{R}$ is modest; however, the FST models will be less accurate than at the smaller filter width. None of the models considered yields particularly accurate predictions for the FSTs, with errors ranging from $10 \%$ to $90 \%$. However, this does not necessarily preclude their use in LES, since from the budgets of the LES equations ( $\$ 4.3)$, the FSTs are an order of magnitude smaller than the largest terms. The sensitivity of flow field and drop evolution to FST errors can only be determined by performing an a posteriori LES study.

\section{Subgrid scale models}

The analysis now turns to evaluating models for the SGS fluxes (defined in (4.8)) appearing in the LES equations (4.18)-(4.20). This activity involves considering several possible functional forms for the fluxes, which contain proportionality coefficients to be determined through calibration on the DNS transitional flow field. Additionally, some of the FST models described in $\S 6.1$ use the SGS variance $\sigma_{S G S}^{2}$, here defined as

$$
\begin{gathered}
\sigma_{S G S}^{2}\left(\tilde{u}_{1}\right)=\tau_{11}, \quad \sigma_{S G S}^{2}\left(\tilde{u}_{2}\right)=\tau_{22}, \quad \sigma_{S G S}^{2}\left(\tilde{u}_{3}\right)=\tau_{33}, \\
\sigma_{S G S}^{2}(\tilde{T})=\widetilde{T^{2}}-(\tilde{T})^{2}, \quad \sigma_{S G S}^{2}\left(\tilde{Y}_{V}\right)=\widetilde{Y_{V} Y_{V}}-\left(\widetilde{Y}_{V}\right)^{2}, \quad \sigma_{S G S}^{2}(\bar{p})=\overline{p^{2}}-(\bar{p})^{2} .
\end{gathered}
$$

The SGS variances have a similar form to the SGS fluxes and we propose to model them in the same way.

\subsection{Functional forms}

We consider here the following three typical SGS models:

(i) The Smagorinsky (SM) model for the SGS fluxes is

$$
\begin{gathered}
\tau_{i j}-\frac{1}{3} \tau_{k k} \delta_{i j}=-C_{S M} \bar{\Delta}^{2} S(\bar{\phi})\left[S_{i j}(\bar{\phi})-\frac{1}{3} S_{k k}(\bar{\phi}) \delta_{i j}\right], \\
\eta_{j}=-C_{S M} \bar{\Delta}^{2} S(\bar{\phi}) \frac{1}{2} \frac{\partial \tilde{Y}_{V}}{\partial x_{j}}, \quad \zeta_{j}=-C_{S M} \bar{\Delta}^{2} S(\bar{\phi}) \frac{1}{2} \frac{\partial \tilde{h}}{\partial x_{j}},
\end{gathered}
$$

where

$$
S^{2}(\phi)=S_{i j}(\phi) S_{i j}(\phi)
$$

To model $\tau_{k k}$, which may be important for compressible turbulence, we use the Yoshizawa (1986) (YO) model

$$
\tau_{k k}=C_{Y O} \bar{\Delta}^{2} S^{2}(\bar{\phi})
$$

The SM model is based on eddy-viscosity considerations which cannot be directly extended to model the $\sigma_{S G S}^{2}$ of $\tilde{T}, \tilde{Y}_{V}$ and $\bar{p}$.

(ii) The gradient (GR) model for the SGS fluxes is

$$
\tau_{i j}=C_{G R} \bar{\Delta}^{2} \frac{\partial \tilde{u}_{i}}{\partial x_{k}} \frac{\partial \tilde{u}_{j}}{\partial x_{k}}, \quad \eta_{j}=C_{G R} \bar{\Delta}^{2} \frac{\partial \tilde{Y}_{V}}{\partial x_{k}} \frac{\partial \tilde{u}_{j}}{\partial x_{k}}, \quad \zeta_{j}=C_{G R} \bar{\Delta}^{2} \frac{\partial \tilde{h}}{\partial x_{k}} \frac{\partial \tilde{u}_{j}}{\partial x_{k}},
$$


while the GR model for $\sigma_{S G S}^{2}(\tilde{\psi})$ is

$$
\sigma_{S G S}^{2}(\tilde{\psi})=C_{G R} \bar{\Delta}^{2} \frac{\partial \tilde{\psi}}{\partial x_{k}} \frac{\partial \tilde{\psi}}{\partial x_{k}} .
$$

Theoretically (i.e. from a Taylor series expansion, see Appendix A), $C_{G R}=1 / 12$ for a cubic top-hat filter.

(iii) The scale-similarity (SS) model for the SGS fluxes is

$$
\tau_{i j}=C_{S S}\left(\widehat{\widetilde{u}}_{i} \tilde{u}_{j}-\widehat{\widetilde{u}}_{i} \widehat{\tilde{u}}_{j}\right), \quad \eta_{j}=C_{S S}\left(\widehat{\widetilde{Y}}_{V} \tilde{u}_{j}-\widehat{\tilde{Y}}_{V} \widehat{\tilde{u}}_{j}\right), \quad \zeta_{j}=C_{S S}\left(\widehat{\tilde{h} \tilde{u}_{j}}-\widehat{\tilde{h}}_{\tilde{\tilde{u}}_{j}}\right),
$$

and the SS model for $\sigma_{S G S}^{2}(\tilde{\psi})$ is

$$
\sigma_{S G S}^{2}(\tilde{\psi})=C_{S S}\left[\left(\widehat{(\tilde{\psi})^{2}}-(\widehat{\tilde{\psi}})^{2}\right],\right.
$$

where the overhat ( $($ ) denotes (unweighted) filtering at the test-filter level $\hat{\Delta} \geqslant \bar{\Delta}$. Scalesimilarity implies that $C_{S S}=1$ for identical grid and test filters (Bardina et al. 1980; Pruett \& Adams 2000), however, practically, its true value depends on the situation under study as well as on the filter type and on the ratio $\hat{\Delta} / \bar{\Delta}$ (see Appendix B). For example, Liu, Meneveau \& Katz (1994) found from analysis of experimental data that for turbulent jets $0.3 \leqslant C_{S S} \leqslant 0.6$, whereas the rigorous derivation of Pruett, Sochacki \& Adams (2001) led to $C_{S S}=a_{2} / c_{2}$ for second-order grid and test filters whose second-order coefficients in the Taylor expansions are $a_{2}$ and $c_{2}$, respectively.

Although generally each SGS quantity may have a model coefficient value different from the others and furthermore, the coefficient may be spatially and temporally varying, for simplicity, in this study, all SGS quantities will use the same value of the model coefficient $\left(C_{S M}, C_{G R}\right.$ or $\left.C_{S S}\right)$ which is taken spatially and temporally constant. In keeping with the LES philosophy of reduced computational effort, more complicated model coefficients are usually considered only after constant coefficients prove inadequate a posteriori.

\subsection{Constant-coefficient calibration}

The availability of a DNS database allows a priori calibration of the constant coefficient appearing in each of the SGS flux models under consideration. The main issue we wish to determine is whether SGS flux models calibrated on SP flows can be applied to TP flows; therefore, in the present paper, a priori analysis is devoted to SP600 and TP600a2, because they have the higher $R e_{0}$ (see table 1). The restriction to a single $R e_{0}$ and the lower $M L_{0}$ is with the intention to 'blind' test the models thus obtained for all cases on an a posteriori basis (Part 2 of this study).

\subsubsection{Constant-coefficient calibration procedure}

The calibration procedure used here consists of three stages:

(i) Calculation of the coefficient for each SGS quantity. To this end, we use LS fits of the SGS quantities to their models to compute the slope, $b$, which would be the constant coefficient, and the correlation, $\mathscr{R}$, according to (3.6) and (3.7). If $\mathscr{X}$ is the model (without the coefficient) and $\mathscr{Y}$ is the SGS quantity, then the slope is the constant coefficient; ideally, $\mathscr{R}$ would be unity.

(ii) Calculation of the coefficient for all SGS quantities. To obtain a single coefficient, we average the slopes for the individual SGS quantities. The standard deviation of these slopes measures their variation from the average. Therefore, the smaller the standard deviation relative to the average slope, the more suitable is the average slope for being the single coefficient for all the SGS quantities. This 


\begin{tabular}{lccccc} 
& \multicolumn{2}{c}{$\bar{\Delta}=4 \Delta x$} & & \multicolumn{2}{c}{$\bar{\Delta}=8 \Delta x$} \\
\cline { 2 - 3 } \cline { 5 - 6 } \multicolumn{1}{c}{ SGS quantity } & SP600 & TP600a 2 & & SP600 & TP600a2 \\
$\bar{\rho}\left(\tau_{11}-\tau_{k k} / 3\right)$ & 0.0513 & 0.0478 & & 0.0500 & 0.0456 \\
$\bar{\rho}\left(\tau_{22}-\tau_{k k} / 3\right)$ & 0.0366 & 0.0362 & & 0.0280 & 0.0304 \\
$\bar{\rho}\left(\tau_{33}-\tau_{k k} / 3\right)$ & 0.0396 & 0.0400 & & 0.0403 & 0.0414 \\
$\bar{\rho} \tau_{12}$ & 0.0681 & 0.0660 & & 0.0710 & 0.0733 \\
$\bar{\rho} \tau_{13}$ & 0.0450 & 0.0436 & & 0.0370 & 0.0399 \\
$\bar{\rho} \tau_{23}$ & 0.0325 & 0.0340 & & 0.0284 & 0.0272 \\
$\bar{\rho} \zeta_{1}$ & 0.0885 & 0.1464 & & 0.0486 & 0.1375 \\
$\bar{\rho} \zeta_{2}$ & 0.1121 & 0.1505 & & 0.1004 & 0.1576 \\
$\bar{\rho} \zeta_{3}$ & 0.0925 & 0.0873 & & 0.0752 & 0.0757 \\
$\bar{\rho} \eta_{1}$ & - & 0.1512 & & - & 0.1464 \\
$\bar{\rho} \eta_{2}$ & - & 0.1536 & & - & 0.1598 \\
$\bar{\rho} \eta_{3}$ & - & 0.0884 & & - & 0.0763 \\
Average slope & 0.0629 & 0.0871 & & 0.0532 & 0.0843 \\
Standard deviation of slopes & 0.0287 & 0.0501 & & 0.0243 & 0.0518 \\
Average correlation & 0.25 & 0.31 & & 0.24 & 0.31
\end{tabular}

TABLE 17. Slopes from least-squares fit of SGS quantities to SGS models, Smagorinsky model.

\begin{tabular}{llllll} 
& \multicolumn{2}{c}{$\bar{\Delta}=4 \Delta x$} & & \multicolumn{2}{c}{$\bar{\Delta}=8 \Delta x$} \\
\cline { 2 - 3 } \multicolumn{1}{c}{$\bar{\rho} \tau_{k k}$} & SP600 & TP600a2 & & SP600 & TP600a2 \\
Slope & 0.3043 & 0.3096 & & 0.3154 & 0.3265 \\
Correlation & 0.81 & 0.79 & & 0.79 & 0.77
\end{tabular}

TABLE 18. Slopes from least-squares fit of SGS quantities to SGS models, Yoshizawa model.

coefficient evaluation by means of the average slope, while not physically rigorous, provides a starting point for LES; in the a posteriori study we will assess whether the constant-coefficient approach, and the values here obtained, are viable for TP LES.

(iii) Calculation of the SGS model coefficient. To obtain a single coefficient, we calculate the average slopes on many filtered flow fields (or realizations of the same flow), and develop the best value for the SGS model coefficient. This coefficient may be found to depend on filter width, $R e_{0}, M_{c, 0}$ flow configuration, etc. The applicability of the coefficient will generally be limited to the types of flow for which it was calibrated, which constitutes the main drawback of the constant-coefficient method. However, the hope of the constant-coefficient LES methodology is that the SGS are somewhat insensitive to the large-scale flow, and that their calibration on a geometrically simple transitional flow will be applicable to a variety of other flows. In this study, the focus is on possible dependence of SGS models on the presence or absence of drops, and on the filter width. This dependence of the coefficient on the flow field is measured by performing $t$-tests of statistical significance on the average slopes obtained in the second stage described above.

\subsubsection{Results of constant-coefficient calibration}

The calibration procedure outlined above is performed for the SM, YO, GR and SS models. For the SS model, two test-filter widths $(\hat{\Delta}=\bar{\Delta}$ and $\hat{\Delta}=2 \bar{\Delta})$ are investigated. The analysis is performed for the SP600 and TP600a2 databases at the transitional states $\left(t^{*}=100\right.$ and $t^{*}=105$, respectively) at two different filter widths $(\bar{\Delta}=4 \Delta x$ and 


\begin{tabular}{lccccc} 
& \multicolumn{2}{c}{$\bar{\Delta}=4 \Delta x$} & & \multicolumn{2}{c}{$\bar{\Delta}=8 \Delta x$} \\
\cline { 2 - 3 } \cline { 5 - 6 } \multicolumn{1}{c}{ SGS quantity } & SP600 & TP600a2 & & SP600 & TP600a2 \\
$\bar{\rho} \tau_{11}$ & 0.1596 & 0.1655 & & 0.1556 & 0.1629 \\
$\bar{\rho} \tau_{22}$ & 0.1645 & 0.1683 & & 0.1694 & 0.1756 \\
$\bar{\rho} \tau_{33}$ & 0.1683 & 0.1723 & & 0.1820 & 0.1870 \\
$\bar{\rho} \tau_{12}$ & 0.1448 & 0.1478 & & 0.1313 & 0.1339 \\
$\bar{\rho} \tau_{13}$ & 0.1479 & 0.1506 & & 0.1334 & 0.1354 \\
$\bar{\rho} \tau_{23}$ & 0.1475 & 0.1503 & & 0.1343 & 0.1367 \\
$\bar{\rho} \zeta_{1}$ & 0.1488 & 0.1464 & & 0.1346 & 0.1345 \\
$\bar{\rho} \zeta_{2}$ & 0.1475 & 0.1450 & & 0.1338 & 0.1324 \\
$\bar{\rho} \zeta_{3}$ & 0.1489 & 0.1463 & & 0.1334 & 0.1319 \\
$\bar{\rho} \eta_{1}$ & - & 0.1468 & & - & 0.1346 \\
$\bar{\rho} \eta_{2}$ & - & 0.1455 & & - & 0.1327 \\
$\bar{\rho} \eta_{3}$ & - & 0.1466 & & - & 0.1322 \\
$\sigma_{S G S}^{2}(\tilde{T})$ & 0.1737 & 0.1758 & & 0.1809 & 0.1828 \\
$\sigma_{S G S}^{2}\left(\tilde{Y}{ }_{V}\right)$ & - & 0.1725 & & - & 0.1784 \\
$\sigma_{S G S}^{2}(\bar{p})$ & 0.1588 & 0.1666 & & 0.1349 & 0.1580 \\
Average slope & 0.1552 & 0.1564 & & 0.1476 & 0.1499 \\
Standard deviation of slopes & 0.0099 & 0.0119 & & 0.0205 & 0.0216 \\
Average correlation & 0.97 & 0.97 & & 0.92 & 0.91
\end{tabular}

TABLE 19. Slopes from least-squares fit of SGS quantities to SGS models, gradient model.

$\bar{\Delta}=8 \Delta x$, see $\S 4.2)$ for a total of 4 data sets. The SGS quantities analysed are $\left\{\bar{\rho} \tau_{i j}\right.$, $\left.\bar{\rho} \zeta_{j}, \sigma_{S G S}^{2}(\tilde{T}), \sigma_{S G S}^{2}(\bar{p})\right\}$ (11 quantities) for case SP600 (for which $\tilde{Y}_{V}=0, \eta_{j}=0$ ) and $\left\{\bar{\rho} \tau_{i j}, \bar{\rho} \zeta_{j}, \bar{\rho} \eta_{j}, \sigma_{S G S}^{2}(\tilde{T}), \sigma_{S G S}^{2}\left(\tilde{Y}_{V}\right), \sigma_{S G S}^{2}(\bar{p})\right\}$ (15 quantities) for case TP600a2. The $\sigma_{S G S}^{2}\left(\tilde{u}_{i}\right)$ are represented as a subset of $\tau_{i j}$. For each model, the slopes for each SGS quantity are presented in tables 17-20, along with the average and the standard deviation of the slopes and the average of the correlations. We apply a two-tailed $t$-test for equality of means to these slopes (at a 5\% level of significance, assuming unequal variances), and present the results of the $t$-tests in table 21 . If the $t$-test indicates that the average slopes are equal, we take the mean of the average slopes (of the appropriate data sets) as the model coefficient. The analysis of the models, including the calibrated coefficients, can be summarized as follows.

(i) Smagorinsky model (table 17) and Yoshizawa model (table 18). The correlation between the SM models and the SGS quantities is poor (0.24 to 0.31$)$, and the large standard deviation is due to the large spread of the individual slopes. The $t$-test indicates that the same coefficient can be applied to all four data sets; the mean value of the average slopes is 0.072 . For $\tau_{k k}$, the YO model (table 18) has a correlation of about 0.8 , which is considerably better than that for the SM model. From the four data sets considered, the mean value is $C_{Y O}=0.314$. This large value of $C_{Y O}$ compared to that of $C_{S M}$ indicates that $\tau_{k k}$ should not be neglected, in contrast to the SGS stress results of Erlebacher et al. (1992) for isotropic turbulence. However, since the Smagorinsky model generally correlates poorly with the SGS quantities, it is not clear whether any benefit would be derived from not neglecting $\tau_{k k}$, which does not alter the average slope or the calibrated coefficient. The calibration of the SM model along with the YO model leads to

$$
C_{S M}=0.072, C_{Y O}=0.314
$$




\begin{tabular}{|c|c|c|c|c|c|c|c|c|}
\hline \multirow[b]{3}{*}{ SGS quantity } & \multicolumn{4}{|c|}{$\hat{\Delta}=\bar{\Delta}$} & \multicolumn{4}{|c|}{$\hat{\Delta}=2 \bar{\Delta}$} \\
\hline & \multicolumn{2}{|c|}{$\bar{\Delta}=4 \Delta x$} & \multicolumn{2}{|c|}{$\bar{\Delta}=8 \Delta x$} & \multicolumn{2}{|c|}{$\bar{\Delta}=4 \Delta x$} & \multicolumn{2}{|c|}{$\bar{\Delta}=8 \Delta x$} \\
\hline & SP600 & TP600a2 & SP600 & TP600a2 & SP600 & TP600a2 & SP600 & TP600a2 \\
\hline $\bar{\rho} \tau_{11}$ & 1.5832 & 1.6691 & 1.9327 & 2.0636 & 0.6114 & 0.6562 & 0.7143 & 0.7818 \\
\hline $\bar{\rho} \tau_{22}$ & 1.6733 & 1.7390 & 2.2583 & 2.3869 & 0.6780 & 0.7163 & 0.9460 & 1.0100 \\
\hline $\bar{\rho} \tau_{33}$ & 1.7341 & 1.7954 & 2.5860 & 2.6523 & 0.7317 & 0.7613 & 1.2425 & 1.2588 \\
\hline $\bar{\rho} \tau_{12}$ & 1.4629 & 1.5364 & 1.6903 & 1.7806 & 0.5508 & 0.5949 & 0.6384 & 0.6679 \\
\hline $\bar{\rho} \tau_{13}$ & 1.5728 & 1.6212 & 1.9217 & 1.9603 & 0.6522 & 0.6744 & 0.8130 & 0.8180 \\
\hline $\bar{\rho} \tau_{23}$ & 1.5864 & 1.6472 & 1.9940 & 2.0627 & 0.6678 & 0.7052 & 0.8991 & 0.9187 \\
\hline $\bar{\rho} \zeta_{1}$ & 1.5317 & 1.4812 & 1.7572 & 1.7383 & 0.5921 & 0.5638 & 0.6444 & 0.6509 \\
\hline $\bar{\rho} \zeta_{2}$ & 1.5340 & 1.4754 & 1.7675 & 1.7472 & 0.5969 & 0.5625 & 0.6438 & 0.6819 \\
\hline $\bar{\rho} \zeta_{3}$ & 1.6009 & 1.5378 & 1.9138 & 1.8865 & 0.6611 & 0.6236 & 0.7833 & 0.8002 \\
\hline $\bar{\rho} \eta_{1}$ & - & 1.4888 & - & 1.7480 & - & 0.5683 & - & 0.6565 \\
\hline $\bar{\rho} \eta_{2}$ & - & 1.4850 & - & 1.7581 & - & 0.5676 & - & 0.6897 \\
\hline $\bar{\rho} \eta_{3}$ & - & 1.5422 & - & 1.8929 & - & 0.6248 & - & 0.8049 \\
\hline$\sigma_{S G S}^{2}(\tilde{T})$ & 1.8006 & 1.8430 & 2.3777 & 2.4825 & 0.7412 & 0.7719 & 0.9442 & 1.0575 \\
\hline$\sigma_{S G S}^{2}\left(\tilde{Y}_{V}\right)$ & - & 1.7918 & - & 2.4081 & - & 0.7439 & - & 1.0229 \\
\hline$\sigma_{S G S}^{2}(\bar{p})$ & 1.4381 & 1.6450 & 1.4324 & 1.8154 & 0.4913 & 0.6211 & 0.4246 & 0.5668 \\
\hline Average slope & 1.5925 & 1.6199 & 1.9665 & 2.0256 & 0.6340 & 0.6504 & 0.7903 & 0.8259 \\
\hline $\begin{array}{l}\text { Standard deviation } \\
\text { of slopes }\end{array}$ & 0.1087 & 0.1263 & 0.3301 & 0.3088 & 0.0749 & 0.0746 & 0.2168 & 0.1919 \\
\hline Average correlation & 0.94 & 0.93 & 0.89 & 0.88 & 0.82 & 0.82 & 0.74 & 0.73 \\
\hline
\end{tabular}

TABLE 20. Slopes from least-squares fit of SGS quantities to SGS models, similarity model.

\begin{tabular}{|c|c|c|c|c|c|c|c|c|}
\hline \multirow[b]{3}{*}{ SGS Model } & \multicolumn{4}{|c|}{ Average slope } & \multicolumn{4}{|c|}{ Average slopes equal? } \\
\hline & \multicolumn{2}{|c|}{$\bar{\Delta} / \Delta x=4$} & \multicolumn{2}{|c|}{$\bar{\Delta} / \Delta x=8$} & \multicolumn{2}{|c|}{ SP600 and TP600a2 } & \multicolumn{2}{|c|}{$\bar{\Delta} / \Delta x=4$ and 8} \\
\hline & SP600 & TP600a2 & SP600 & TP600a2 & $\bar{\Delta} / \Delta x=4$ & $\bar{\Delta} / \Delta x=8$ & SP600 & TP600a2 \\
\hline SM & 0.0629 & 0.0871 & 0.0532 & 0.0843 & Yes & Yes & Yes & Yes \\
\hline GR & 0.1552 & 0.1564 & 0.1476 & 0.1499 & Yes & Yes & Yes & Yes \\
\hline SS1 & 1.5925 & 1.6199 & 1.9665 & 2.0256 & Yes & Yes & No & No \\
\hline SS2 & 0.6340 & 0.6504 & 0.7903 & 0.8259 & Yes & Yes & No & No \\
\hline
\end{tabular}

TABLE 21. Average slopes and $t$-test for equality of average slopes, comparing SP600 to TP600a 2 and $\bar{\Delta}=4 \Delta x$ to $\bar{\Delta}=8 \Delta x$. SGS models are: SM=Smagorinsky, GR $=$ gradient, $\mathrm{SS}=$ scale-similarity model with $\hat{\Delta}=\bar{\Delta}(\mathrm{SS} 1)$ or $\hat{\Delta}=2 \bar{\Delta}$ (SS2). See tables 17,19 and 20 for the distributions used to calculate the average slopes.

The value obtained here of $C_{S M}=0.072$ is higher than $C_{S M}=0.17^{2} \simeq 0.029$ used by Vreman, Geurts \& Kuerten (1997) for the SGS stresses, which was found to be too dissipative; the calibrated values will lead to a SM model that is even more dissipative.

(ii) Gradient model (table 19). The correlation between models and SGS quantities is excellent (0.91 to 0.97). From the $t$-test, the average slopes are equal for all four data sets, with a mean value across the four data sets of 0.152 . The calibration leads to the GR model coefficient as

$$
C_{G R}=0.152 \text {. }
$$

(iii) Similarity model (table 20). The correlation between models and SGS quantities ranges from good to excellent $(0.73$ to 0.94$)$, with better correlation for $\hat{\Delta}=\bar{\Delta}$. From the $t$-test, the average slopes are statistically equal when comparing SP600 to TP600a2, 
but not when comparing $\bar{\Delta}=4 \Delta x$ to $\bar{\Delta}=8 \Delta x$. Therefore, the SS model calibration yields

$$
\begin{aligned}
& \hat{\Delta}=\bar{\Delta}: C_{S S}=1.606 \text { for } \bar{\Delta}=4 \Delta x, C_{S S}=1.996 \text { for } \bar{\Delta}=8 \Delta x \\
& \hat{\Delta}=2 \bar{\Delta}: C_{S S}=0.642 \text { for } \bar{\Delta}=4 \Delta x, C_{S S}=0.808 \text { for } \bar{\Delta}=8 \Delta x
\end{aligned}
$$

Since the theoretical model coefficient is unity, the closest value to theory is attained with $\bar{\Delta}=8 \Delta x$ and $\hat{\Delta}=2 \bar{\Delta}$. The coefficients at $\hat{\Delta}=\bar{\Delta}$ are about 2.5 times larger than those at $\hat{\Delta}=2 \bar{\Delta}$ for the same $\bar{\Delta}$, so there is a strong coefficient dependence on $\hat{\Delta} / \bar{\Delta}$. On the other hand, the coefficients at $\bar{\Delta}=4 \Delta x$ are about $80 \%$ of those at $\bar{\Delta}=8 \Delta x$, so the coefficient dependence on $\bar{\Delta} / \Delta x$ is much weaker than that on $\hat{\Delta} / \bar{\Delta}$. It can be analytically shown that there should be a coefficient dependence on $\hat{\Delta} / \bar{\Delta}$ (Pruett et al. 2001; see Appendix B) such that $C_{S S}$ decreases with increasing $\hat{\Delta} / \bar{\Delta}$. However, the coefficient dependence on $\bar{\Delta} / \Delta x$ being weak, suggests that the flow field is not quite scale-similar, perhaps because the database represents a transitional state rather than the fully turbulent regime (which is currently unachievable in DNS).

\subsubsection{Constant-coefficient calibration conclusions}

These results are consistent with the a priori SGS stress investigation of Pruett \& Adams (2000) for the decay of isotropic turbulence in which the SM model correlated poorly with the exact stresses and the SS model correlated remarkably well, particularly when the grid and test filter were identical. However, correlations for the GR model are here much better when compared to those obtained by Pruett \& Adams (2000) (0.9 compared to 0.6); this may be due to their use of the same form of the GR model as has been analysed here that applies only to cubic top-hat filters (see Appendix A), and which is inconsistent with their filter (one-parameter Pade-type filter). At this juncture, the prospects for successful TP LES using the SM model seem poor, since drop evolution in TP flows is strongly dependent on the local conditions which are poorly captured by this model. The model calibration exercise also suggests that, to overcome deficiencies in the GR or SS model that have been found in some a posteriori studies (e.g. Vreman et al. 1997, who used the theoretical coefficients $C_{S S}=1, C_{G R}=1 / 12$ for the SGS stresses), it may be more fruitful to adjust $C_{G R}$ or $C_{S S}$ rather than add the SM model (leading to 'mixed' models) and then attempt to adjust the SM coefficient.

The most encouraging result of this calibration is the suggestion that coefficients calibrated on compressible SP flow are statistically equivalent to those for TP flow with evaporating drops, implying that SGS models already developed for compressible SP flows may be applied to the SGS fluxes in TP flows. However, since the a priori study does not include either the effect of the flow-field evolution on the drops or the interaction among all scales inherent in turbulence, a posteriori studies are needed to bear out this implication.

\section{Summary and conclusions}

We have conducted direct numerical simulations (DNS) of a two-phase (TP) temporal mixing layer laden with evaporating drops as a precursor to conducting large eddy simulations (LES) of this flow. The DNS provide a database on which $a$ priori analyses of the LES equations can be conducted. The gas-phase was computed in an Eulerian frame using the compressible Navier-Stokes equations along with conservation equations for total energy, carrier gas mass and vapour mass and a perfect gas equation of state. The drops were individually tracked in a Lagrangian 
frame, with their effect on the flow being modelled through source terms in the gas-phase equations. The resolution of the gas phase was adequate for a single-phase (SP) flow. Initially, the gas phase consisted solely of the carrier gas species and, for TP layers, drops were located in the lower stream only and were colder than the gas phase. For the two initial Reynolds numbers (500 and 600) considered, the evolution of SP (null mass loading) and TP (mass loadings of 0.2 and 0.5 ) layers was simulated to attain a transitional state for each layer.

For the TP layers, the vorticity, temperature and vapour mass fraction fields at transition were qualitatively similar regardless of mass loading. In particular, the temperature of the gas in the initially drop-laden stream diminished to be close and sometimes inferior to the initial drop temperature. The range and distribution of the vapour mass fraction at transition showed sensitivity, but no strong effect of the initial mass loading for mass loadings of 0.2 or 0.5 , and the increasing product thickness showed that the layer drops were still evaporating (i.e. the layer was not saturated). Regions of high drop number density occurred in low-vorticity locations and exhibited a complex small-scale organization.

In accord with our stated goal of conducting consistent LES, in which both the grid resolution and the number of tracked drops will be reduced compared to the DNS, we derived the LES equations applicable to TP flows with evaporating drops. These LES equations were derived by spatially filtering the DNS equations, leading to unclosed terms that could not be computed directly from the filtered flow field. By invoking various assumptions, which were validated on the filtered DNS database, and considering the magnitude of the terms in the LES equations at transition, we obtained a hierarchy of terms to guide the simplification of the LES equations and reduce the modelling requirements. In particular, we found that many filtered gasphase quantities are quantitatively close to their counterparts as calculated using the same functional form as in the DNS, but from the filtered flow field. The unclosed terms that were found to be of comparable magnitude at transitional states of TP flow and that remained to be modelled were the filtered source terms (FSTs) and the subgrid scale (SGS) fluxes.

The importance of the source terms was further analysed by deriving the irreversible entropy production (i.e. dissipation) expression for TP flows with phase change and calculating the dissipation budget at the DNS, LES and subgrid scales. At a given initial Reynolds number, the dissipation was found to increase with increasing mass loading, while for a given mass loading, the dissipation decreased with increasing Reynolds number; this behaviour showed the need for robust turbulence models that can accommodate a range of mass loadings. In contrast to SP flows where the dissipation was governed by viscous effects, for the TP flows studied here, the contribution to the dissipation was dominated by terms arising from drop-source terms. The two most important contributions were due to the energy source term and the chemical potential of the vapour emanating from the drops, and were positive and negative, respectively, at the DNS and LES scales, but negative and positive, respectively, at the SGS. At all scales, these two contributions exceeded the contribution from the viscous stresses, and furthermore, for at least one filtered field, the average of the dissipation term originating from the momentum source contribution rivalled the average viscous dissipation in magnitude, indicating the importance, in LES, of the FST models for portraying the correct physics of the flow.

The LES models evaluated a priori on the DNS database were of two types: those for the FSTs representing the effect of the drops on the filtered flow field; and those for the SGS fluxes arising from filtering the convective terms in the gas-phase 
DNS equations. The unfiltered flow field is required for the calculation of drop-based quantities, but would not be available in LES; therefore, various approximations were attempted for it, namely, the filtered flow field and the filtered flow field plus corrections based on the SGS variances. All of the FST models were found to overestimate FSTs, with the relative error of modelling the unfiltered flow field compared to that of using computational drops showing a complex dependence on filter width and number of computational drops. Generally, as the number of computational drops decreased, the performance of the models deteriorated, and eventually the modelling error became independent of the filter size.

To complete the LES modelling, constant-coefficient Smagorinsky, gradient and scale-similarity models for SGS quantities were assessed and calibrated on the DNS database. The gradient and scale-similarity models showed excellent correlation with the SGS fluxes and variances, while the Smagorinsky model (possible only for SGS fluxes) performed poorly. Calibrated values of the SGS model coefficients were computed for various filtered flow fields; the values were compared for transitional states generated from SP and TP DNS which had the same initial Reynolds number and grid size and resolution. For all SGS flux models, these coefficients were statistically equivalent when computed on SP or TP flows. Additionally, for the Smagorinsky and gradient model, these coefficients were statistically insensitive to the filter width, whereas for the scale-similarity model, the coefficients depended on the filter widths used.

Although some of the models presented seem promising, the true test of their appropriateness will be in a posteriori studies. Such studies are needed to determine whether these models will lead to the proper evolution of the filtered flow field and of the computational drops, and to appropriate interaction between the resolved and modelled (i.e. SGS) flow field. Of particular interest will be the behaviour of the models for the FSTs as the number of computational drops is decreased.

This work was conducted at the Jet Propulsion Laboratory (JPL) of the California Institute of Technology, and was sponsored by the US Department of Energy (contract monitors were N. Rossmeissl, Headquarters and D. Hooker, Golden Center) under an agreement with the National Aeronautics and Space Administration. Computations were performed on the SGI Origin2000 at the JPL Supercomputing Center.

\section{Appendix A. Derivation of gradient model}

The gradient model (Clark et al. 1979) can be derived starting from the Taylor series expansion for a variable $\psi_{i}$,

$$
\psi_{i}(\boldsymbol{x})=\psi_{i}\left(\boldsymbol{x}_{0}\right)+\frac{\partial \psi_{i}}{\partial x_{k}}\left(\boldsymbol{x}_{0}\right) I_{k}+\frac{1}{2} \frac{\partial^{2} \psi_{i}}{\partial x_{k} \partial x_{l}}\left(\boldsymbol{x}_{0}\right) I_{k l}+O\left(\bar{\Delta}^{3}\right),
$$

where $I_{k}=\left(x_{k}-x_{k_{0}}\right)$ and $I_{k l}=\left(x_{k}-x_{k_{0}}\right)\left(x_{l}-x_{l_{0}}\right)$. Filtering this expansion leads to

$$
\bar{\psi}_{i}=\psi_{i}\left(\boldsymbol{x}_{0}\right)+\frac{\partial \psi_{i}}{\partial x_{k}}\left(\boldsymbol{x}_{0}\right) \bar{I}_{k}+\frac{1}{2} \frac{\partial^{2} \psi_{i}}{\partial x_{k} \partial x_{l}}\left(\boldsymbol{x}_{0}\right) \bar{I}_{k l}+O\left(\bar{\Delta}^{3}\right) .
$$

Multiplying the series expansions for the variables $\psi_{i}$ and $\psi_{j}$, then filtering yields

$$
\begin{aligned}
& \overline{\psi_{i} \psi_{j}}=\psi_{i}\left(\boldsymbol{x}_{0}\right) \psi_{j}\left(\boldsymbol{x}_{0}\right)+\left[\psi_{i}\left(\boldsymbol{x}_{0}\right) \frac{\partial \psi_{j}}{\partial x_{k}}\left(\boldsymbol{x}_{0}\right)+\psi_{j}\left(\boldsymbol{x}_{0}\right) \frac{\partial \psi_{i}}{\partial x_{k}}\left(\boldsymbol{x}_{0}\right)\right] \overline{\boldsymbol{I}}_{k} \\
& +\left\{\frac{1}{2}\left[\psi_{i}\left(\boldsymbol{x}_{0}\right) \frac{\partial^{2} \psi_{j}}{\partial x_{k} \partial x_{l}}\left(\boldsymbol{x}_{0}\right)+\psi_{j}\left(\boldsymbol{x}_{0}\right) \frac{\partial^{2} \psi_{i}}{\partial x_{k} \partial x_{l}}\left(\boldsymbol{x}_{0}\right)\right]+\frac{\partial \psi_{i}}{\partial x_{k}}\left(\boldsymbol{x}_{0}\right) \frac{\partial \psi_{j}}{\partial x_{l}}\left(\boldsymbol{x}_{0}\right)\right\} \overline{\boldsymbol{I}}_{k l} .
\end{aligned}
$$


Multiplying the series expansions for the variables $\bar{\psi}_{i}$ and $\bar{\psi}_{j}$ yields

$$
\begin{aligned}
& \bar{\psi}_{i} \bar{\psi}_{j}=\psi_{i}\left(\boldsymbol{x}_{0}\right) \psi_{j}\left(\boldsymbol{x}_{0}\right)+\left[\psi_{i}\left(\boldsymbol{x}_{0}\right) \frac{\partial \psi_{j}}{\partial x_{k}}\left(\boldsymbol{x}_{0}\right)+\psi_{j}\left(\boldsymbol{x}_{0}\right) \frac{\partial \psi_{i}}{\partial x_{k}}\left(\boldsymbol{x}_{0}\right)\right] \bar{I}_{k} \\
& +\frac{1}{2}\left[\psi_{i}\left(\boldsymbol{x}_{0}\right) \frac{\partial^{2} \psi_{j}}{\partial x_{k} \partial x_{l}}\left(\boldsymbol{x}_{0}\right)+\psi_{j}\left(\boldsymbol{x}_{0}\right) \frac{\partial^{2} \psi_{i}}{\partial x_{k} \partial x_{l}}\left(\boldsymbol{x}_{0}\right)\right] \overline{\boldsymbol{I}}_{k l}+\frac{\partial \psi_{i}}{\partial x_{k}}\left(\boldsymbol{x}_{0}\right) \frac{\partial \psi_{j}}{\partial x_{l}}\left(\boldsymbol{x}_{0}\right) \bar{I}_{k} \bar{I}_{l}
\end{aligned}
$$

Then, the general form of the gradient model, valid for any filter, is

$$
\overline{\psi_{i} \psi_{j}}-\bar{\psi}_{i} \bar{\psi}_{j}=\frac{\partial \psi_{i}}{\partial x_{k}} \frac{\partial \psi_{j}}{\partial x_{l}}\left[\bar{I}_{k l}-\bar{I}_{k} \bar{I}_{l}\right],
$$

where (A 3)-(A 5) are accurate to $O\left(\bar{\Delta}^{3}\right)$. Now, for a top-hat filter

$$
\bar{\psi}\left(\boldsymbol{x}_{0}\right)=\frac{1}{V_{f}} \int_{V_{f}} \psi(\boldsymbol{x}) \mathrm{d} V,
$$

where $V_{f}$ is the filtering volume. Then, if $x_{0}$ is the centroid of the filtering volume, $\bar{I}_{k}=0$. The terms $\bar{I}_{k l}$ are the moments of inertia, which in the case of a cubic top-hat filter with symmetrical filtering volumes are $\bar{\Delta}^{2} \delta_{k l} / 12$ leading to

$$
\overline{\psi_{i} \psi_{j}}-\bar{\psi}_{i} \bar{\psi}_{j}=\frac{1}{12} \bar{\Delta}^{2} \frac{\partial \psi_{i}}{\partial x_{k}} \frac{\partial \psi_{j}}{\partial x_{k}} .
$$

Approximating the $\psi_{i}$ derivatives by $\bar{\psi}_{i}$ derivatives leads to the gradient model for a cubic top-hat filter as

$$
\overline{\psi_{i} \psi_{j}}-\bar{\psi}_{i} \bar{\psi}_{j}=C_{G R} \bar{\Delta}^{2} \frac{\partial \bar{\psi}_{i}}{\partial x_{k}} \frac{\partial \bar{\psi}_{j}}{\partial x_{k}} .
$$

This model is applied to the Favre-filtered quantities as

$$
\widetilde{\psi_{i} \psi_{j}}-\tilde{\psi}_{i} \tilde{\psi}_{j}=C_{G R} \bar{\Delta}^{2} \frac{\partial \tilde{\psi}_{i}}{\partial x_{k}} \frac{\partial \tilde{\psi}_{j}}{\partial x_{k}} .
$$

This expression can be derived from (A 5) by replacing the unweighted filtering with Favre-filtering, namely

$$
\widetilde{\psi_{i} \psi_{j}}-\tilde{\psi}_{i} \tilde{\psi}_{j}=\frac{\partial \psi_{i}}{\partial x_{k}} \frac{\partial \psi_{j}}{\partial x_{l}}\left[\tilde{I}_{k l}-\tilde{I}_{k} \tilde{I}_{l}\right],
$$

then using $\tilde{I}_{k} \simeq \bar{I}_{k}$ and $\tilde{I}_{k l} \simeq \bar{I}_{k l}$ and approximating the $\psi_{i}$ derivatives by $\tilde{\psi}_{i}$ derivatives.

The model coefficient $C_{G R}$ can be expected to be larger than its theoretical value, because, since $\bar{\psi}_{i}$ is smoother than $\psi_{i}, \partial \bar{\psi}_{i} / \partial x_{k}$ will have a smaller magnitude than $\partial \psi_{i} / \partial x_{k}$.

\section{Appendix B. Test-filter ratio dependence of scale-similarity model}

The scale similarity model is (Bardina et al. 1980)

$$
{\overline{\psi_{i} \psi_{j}}}_{-\bar{\psi}_{i}} \bar{\psi}_{j}=C_{S S}\left(\widehat{\bar{\psi}}_{i} \bar{\psi}_{j}-\widehat{\bar{\psi}}_{i} \widehat{\bar{\psi}}_{j}\right)
$$

where the overhat $(\widehat{ })$ denotes filtering at the test-filter width $\hat{\Delta} \geqslant \bar{\Delta}$. For clarity, the derivation that follows uses the unweighted filter. The application to Favre-filtered quantities is through replacing the unweighted filter with the density-weighted filter, e.g.

$$
{\widetilde{\psi_{i} \psi_{j}}}_{-}-\tilde{\psi}_{i} \tilde{\psi}_{j}=C_{S S}\left({\widetilde{\tilde{\psi}_{i} \tilde{\psi}_{j}}}_{-}-\widehat{\tilde{\psi}}_{i} \widehat{\tilde{\psi}}_{j}\right)
$$

where re-filtering is always unweighted. 
From (A 2),

$$
\begin{aligned}
& \bar{\psi}_{i}\left(\boldsymbol{x}_{0}\right)=\psi_{i}\left(\boldsymbol{x}_{0}\right)+\frac{\partial \psi_{i}}{\partial x_{m}}\left(\boldsymbol{x}_{0}\right) \bar{I}_{m}+\frac{1}{2} \frac{\partial^{2} \psi_{i}}{\partial x_{m} \partial x_{n}}\left(\boldsymbol{x}_{0}\right) \bar{I}_{m n}+O\left(\bar{\Delta}^{3}\right) \\
& \hat{\psi}_{i}\left(\boldsymbol{x}_{0}\right)=\psi_{i}\left(\boldsymbol{x}_{0}\right)+\frac{\partial \psi_{i}}{\partial x_{m}}\left(\boldsymbol{x}_{0}\right) \hat{I}_{m}+\frac{1}{2} \frac{\partial^{2} \psi_{i}}{\partial x_{m} \partial x_{n}}\left(\boldsymbol{x}_{0}\right) \hat{I}_{m n}+O\left(\bar{\Delta}^{3}\right) .
\end{aligned}
$$

Then, to $O\left(\bar{\Delta}^{3}\right)$,

$$
\begin{gathered}
\widehat{\bar{\psi}}_{i}=\bar{\psi}_{i}\left(\boldsymbol{x}_{0}\right)+\frac{\partial \bar{\psi}_{i}}{\partial x_{m}}\left(\boldsymbol{x}_{0}\right) \hat{I}_{m}+\frac{1}{2} \frac{\partial^{2} \bar{\psi}_{i}}{\partial x_{m} \partial x_{n}}\left(\boldsymbol{x}_{0}\right) \hat{I}_{m n}, \\
\widehat{\bar{\psi}}_{i} \bar{\psi}_{j}=\left(\bar{\psi}_{i} \bar{\psi}_{j}\right)\left(\boldsymbol{x}_{0}\right)+\frac{\partial\left(\bar{\psi}_{i} \bar{\psi}_{j}\right)}{\partial x_{m}}\left(\boldsymbol{x}_{0}\right) \hat{I}_{m}+\frac{1}{2} \frac{\partial^{2}\left(\bar{\psi}_{i} \bar{\psi}_{j}\right)}{\partial x_{m} \partial x_{n}}\left(\boldsymbol{x}_{0}\right) \hat{I}_{m n}, \\
\widehat{\bar{\psi}}_{i} \widehat{\bar{\psi}}_{j}=\bar{\psi}_{i}\left(\boldsymbol{x}_{0}\right) \bar{\psi}_{j}\left(\boldsymbol{x}_{0}\right)+\frac{\partial\left(\bar{\psi}_{i} \bar{\psi}_{j}\right)}{\partial x_{m}}\left(\boldsymbol{x}_{0}\right) \hat{I}_{m}+\frac{\partial \bar{\psi}_{i}}{\partial x_{m}}\left(\boldsymbol{x}_{0}\right) \frac{\partial \bar{\psi}_{j}}{\partial x_{n}}\left(\boldsymbol{x}_{0}\right) \hat{I}_{m} \hat{I}_{n} \\
+\frac{1}{2}\left[\bar{\psi}_{i}\left(\boldsymbol{x}_{0}\right) \frac{\partial^{2} \bar{\psi}_{j}}{\partial x_{m} \partial x_{n}}\left(\boldsymbol{x}_{0}\right)+\bar{\psi}_{j}\left(\boldsymbol{x}_{0}\right) \frac{\partial^{2} \bar{\psi}_{i}}{\partial x_{m} \partial x_{n}}\left(\boldsymbol{x}_{0}\right)\right] \hat{I}_{m n}, \\
{\widehat{\bar{\psi}}, \bar{\psi}_{j}}-\widehat{\bar{\psi}}_{i} \widehat{\bar{\psi}}_{j}=\frac{\partial \bar{\psi}_{i}}{\partial x_{m}} \frac{\partial \bar{\psi}_{j}}{\partial x_{n}}\left[\hat{I}_{m n}-\hat{I}_{m} \hat{I}_{n}\right] .
\end{gathered}
$$

From (B 1),

$$
C_{S S}=\frac{\left({\overline{\psi_{i} \psi_{j}}}-\bar{\psi}_{i} \bar{\psi}_{j}\right)}{\left(\widehat{\bar{\psi}}_{i} \bar{\psi}_{j}-\widehat{\bar{\psi}}_{i} \widehat{\bar{\psi}}_{j}\right)} \text { if }\left(\widehat{\bar{\psi}}_{i} \bar{\psi}_{j}-\widehat{\bar{\psi}}_{i} \widehat{\bar{\psi}}_{j}\right) \neq 0 .
$$

Using (A 5) and (B 8),

$$
C_{S S}=\frac{\left(\frac{\partial \psi_{i}}{\partial x_{k}} \frac{\partial \psi_{j}}{\partial x_{l}}\right)}{\left(\frac{\partial \bar{\psi}_{i}}{\partial x_{m}} \frac{\partial \bar{\psi}_{j}}{\partial x_{n}}\right)} \frac{\left[\bar{I}_{k l}-\bar{I}_{k} \bar{I}_{l}\right]}{\left[\hat{I}_{m n}-\hat{I}_{m} \hat{I}_{n}\right]}
$$

For symmetrical filtering volumes $\bar{I}_{k}=0$ and $\hat{I}_{m}=0$; then assuming $\left(\partial \psi_{i} /\right.$ $\left.\partial x_{k}\right)\left(\partial \psi_{j} / \partial x_{l}\right) \simeq\left(\partial \bar{\psi}_{i} / \partial x_{k}\right)\left(\partial \bar{\psi}_{j} / \partial x_{l}\right)$ leads to

$$
C_{S S} \simeq \bar{I}_{k l} / \hat{I}_{m n}
$$

In the case of a cubic top-hat filter, this would be

$$
C_{S S} \simeq \bar{\Delta}^{2} / \hat{\Delta}^{2}=1 /(\hat{\Delta} / \bar{\Delta})^{2} .
$$

This analysis shows the dependence of $C_{S S}$ on the test-filter to grid-filter ratio. However, it might not predict $C_{S S}$ because, generally, $\partial \bar{\psi}_{i} / \partial x_{k}$ is not a good approximation to $\partial \psi_{i} / \partial x_{k}$. (From (A 2), the error in the approximation is proportional to $\partial^{2} \psi_{i} /\left(\partial x_{k} \partial x_{l}\right)$ which is usually not small in a turbulent flow field.)

\section{REFERENCES}

Ayyub, B. \& McCuen, R. 1997 Probability, Statistics and Reliability for Engineers. CRC Press, Boca Raton, Florida.

Bardina, J., Ferziger, J. \& Reynolds, W. 1980 Improved subgrid scale models for large eddy simulation. Tech. Rep. 80-1357. AIAA.

Boivin, M., Simonin, O. \& SQuires, K. 1998 Direct numerical simulation of turbulence modulation by particles in isotropic turbulence. J. Fluid Mech. 375, 235-263. 
Boivin, M., Simonin, O. \& Squires, K. 2000 On the prediction of gas-solid flows with two-way coupling using large eddy simulation. Phys. Fluids 12, 2080-2090.

Clark, R., Ferziger, J. \& Reynolds, W. 1979 Evaluation of subgrid-scale models using an accurately simulated turbulent flow. J. Fluid Mech. 91, 1-16.

Crowe, C., Chung, J. \& Troutt, T. 1998 Particle mixing in free shear flows. Prog. Energy Combust. Sci. 14, 171-194.

Deutsch, E. \& Simonin, O. 1991 Large eddy simulation applied to the modelling of particulate transport coefficients in turbulent two-phase flows. In Eighth Symposium on Turbulent Shear Flows, pp. 10-1:1-6.

Erlebacher, G., Hussaini, M., Speziale, C. \& Zang, T. 1992 Toward the large-eddy simulation of compressible turbulent flows. J. Fluid Mech. 238, 155-185.

Fleckhaus, D., Hishida, K. \& Maeda, M. 1987 Effect of laden solid particles on the turbulent flow structure of a round free jet. Exps. Fluids 5, 323-333.

FureBY, C. 1996 On subgrid scale modeling in large eddy simulations of compressible fluid flow. Phys. Fluids 8, 1301-1311.

Gore, R. A. \& Crowe, C. 1989 Effect of particle size on modulating turbulence intensity. Intl J. Multiphase Flow 286, 229-255.

Hardalupas, Y., Taylor, A. \& Whitelaw, J. 1989 Velocity and particle-flux characteristics of turbulent particle-laden jets. Proc. R. Soc. Lond. A 426, 31-78.

Hetsroni, G. \& Sokolov, M. 1971 Distribution of mass, velocity, and intensity of turbulence in a two-phase turbulent jet. Trans. ASME: J. Appl. Mech. July, 315-327.

Hirshfelder, J., Curtis, C. \& Bird, R. 1954 Molecular Theory of Gases and Liquids. John Wiley.

Kennedy, C. \& CARpenter, M. 1994 Several new numerical methods for compressible shear layer simulations. Appl. Numer. Maths 14, 397-433.

Le ClercQ, P. \& Bellan, J. 2003 Direct numerical simulation of a transitional temporal mixing layer laden with multicomponent-fuel evaporating drops using continuous thermodynamics. Phys. Fluids (submitted).

LEVY, Y. \& LocKwoOD, F. 1981 Velocity measurements in a particle laden turbulent free jet. Combust. Flame 40, 333-339.

LiU, S., Meneveau, C. \& Katz, J. 1994 On the properties of similarity subgrid-scale models as deduced from measurements in a turbulent jet. J. Fluid Mech. 275, 83-119.

Martin, M., Piomelli, U. \& Candler, G. 2000 Subgrid-scale models for compressible large-eddy simulations. Theor. Comput. Fluid Dyn. 13, 361-376.

MashaYeK, F. 1998 a Direct numerical simulations of evaporating droplet dispersion in forced low-Mach-number turbulence. Intl J. Heat Mass Transfer 41, 2601-2617.

MashaYeK, F. $1998 b$ Droplet-turbulence interactions in low-Mach-number homogeneous shear two-phase flows. J. Fluid Mech. 367, 163-203.

MashaYeK, F. \& JABERI, F. 1999 Particle dispersion in forced isotropic low-Mach-number turbulence. Intl J. Heat Mass Transfer 42, 2823-2836.

Miller, R. \& Bellan, J. 1999 Direct numerical simulation of a confined three-dimensional gas mixing layer with one evaporating hydrocarbon-droplet laden stream. J. Fluid Mech. 384, 293-338.

Miller, R. \& Bellan, J. 2000 Direct numerical simulation and subgrid analysis of a transitional droplet laden mixing layer. Phys. Fluids 12, 650-671.

Modarress, D., Tan, H. \& Elgobashi, S. 1984 Two-component LDA measurement in a two-phase turbulent jet. AIAA J. 22, 624-630.

Moser, R. \& Rogers, M. 1991 Mixing transition and the cascade to small scales in a plane mixing layer. Phys. Fluids A 3, 1128-1134.

OKong'O, N. \& Bellan, J. 2000 A priori subgrid analysis of temporal mixing layers with evaporating droplets. Phys. Fluids 12, 1573-1591.

OKong'o, N., Knight, D. \& Zhou, G. 2000 Large eddy simulations using an unstructured grid compressible Navier-Stokes algorithm. Intl J. Comput. Fluid Dyn. 13, 303-326.

Poinsot, T. \& LeLE, S. 1992 Boundary conditions for direct simulations of compressible viscous flows. J. Comput. Phys. 101, 104-129.

Popper, J., Abuaf, N. \& Hetsroni, G. 1974 Velocity measurements in a two-phase turbulent jet. Intl J. Multiphase Flow 1, 715-726. 
Pruett, C. \& Adams, N. 2000 A priori analyses of three subgrid-scale models for one-parameter families of filters. Phys. Fluids 12, 1133-1142.

Pruett, C., Sochacki, J. \& Adams, N. 2001 On Taylor-series expansions of residual stress. Phys. Fluids 13, 2578-2589.

RÉVEILLON, J. \& VERVISCH, L. 2000 Spray vaporization in nonpremixed turbulent combustion modeling: a single drop model. Combust. Flame 121, 75-90.

Simonin, O., Deutsch, E. \& Boivin, M. 1993 Large eddy simulation and second-moment closure of particle fluctuating motion in two-phase turbulent shear flows. Turbulent Shear Flows vol. 9, pp. 85-115.

Smagorinksy, J. 1993 Some historical remarks on the use of nonlinear viscosities. In Large Eddy Simulation of Complex Engineering and Geophysical Flows (ed. B. Galperin \& S. Orszag), chap. 1, pp. 3-36. Cambridge University Press.

Tsuji, Y., Morikawa, Y., Tanaka, T., Karimine, K. \& Nishida, S. 1988 Measurement of an axisymmetric jet laden with coarse particles. Intl J. Multiphase Flow 14, 565-574.

UijtTewaAl, W. \& Oliemans, R. 1996 Particle dispersion and deposition in direct numerical and large eddy simulations of vertical pipe flows. Phys. Fluids 8, 2590-2604.

Vreman, B., Geurts, B. \& Kuerten, H. 1995 A priori tests of large eddy simulation of the compressible plane mixing layer. J. Engng Maths 29, 299-327.

Vreman, B., Geurts, B. \& Kuerten, H. 1997 Large-eddy simulation of the turbulent mixing layer. J. Fluid Mech. 339, 357-390.

WANG, Q. \& SQuires, K. 1996 Large eddy simulations of particle-laden turbulent channel flow. Phys. Fluids 8, 1207-1223.

Williams, F. 1965 Combustion Theory. Addison-Wesley.

Yamamoto, Y., Ротthoff, M., Tanaka, T., Kajishima, T. \& Tsuji, Y. 2001 Large-eddy simulation of turbulent gas-particle flow in a vertical channel: effect of considering inter-particle collisions. J. Fluid Mech. 442, 303-334.

Yoshizawa, A. 1986 Statistical theory for compressible turbulent shear flows, with the application to subgrid modeling. Phys. Fluids 29, 2152-2164. 
